\title{
Observations of the Icy Universe
}

\author{
A. C. Adwin Boogert \\ Universities Space Research Association, Stratospheric Observatory for Infrared \\ Astronomy, NASA Ames Research Center, MS 232-11, Moffett Field, CA 94035, \\ USA (acaboogert@alumni.caltech.edu)
}

\author{
Perry A. Gerakines \\ Astrochemistry Laboratory, Solar System Exploration Division, NASA Goddard \\ Space Flight Center, Greenbelt, MD 20771, USA
}

\section{Douglas C. B. Whittet}

Department of Physics, Applied Physics and Astronomy and New York Center for Astrobiology, Rensselaer Polytechnic Institute, 110 Eighth Street, Troy, NY 12180, USA

Key Words interstellar ices, astrochemistry, volatiles, interstellar molecules, cometary ices, infrared absorption

\begin{abstract}
Freeze-out of the gas phase elements onto cold grains in dense interstellar and circumstellar media builds up ice mantles consisting of molecules that are mostly formed in situ $\left(\mathrm{H}_{2} \mathrm{O}\right.$, $\mathrm{NH}_{3}, \mathrm{CO}_{2}, \mathrm{CO}, \mathrm{CH}_{3} \mathrm{OH}$, and more). This review summarizes the detected infrared spectroscopic ice features and compares the abundances across Galactic, extragalactic, and solar system environments. A tremendous amount of information is contained in the ice band profiles. Laboratory experiments play a critical role in the analysis of the observations. Strong evidence is found for distinct ice formation stages, separated by $\mathrm{CO}$ freeze out at high densities. The ice bands have proven to be excellent probes of the thermal history of their environment. The evidence for the long-held idea that processing of ices by energetic photons and cosmic rays produces complex molecules is weak. Recent state of the art observations show promise for much progress in this area with planned infrared facilities.
\end{abstract}

\section{CONTENTS}

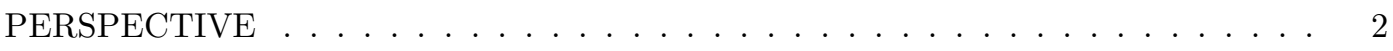

ICES ACROSS THE ELECTROMAGNETIC SPECTRUM . . . . . . . . . . . . 4

Ice Mapping . . . . . . . . . . . . . . . . . . . . . . . . 5

LABORATORY EXPERIMENTS . . . . . . . . . . . . . . . . . 10

ICE BAND PROFILES . . . . . . . . . . . . . . . . . . . . . . 11

The $3.0 \mu \mathrm{m} \mathrm{H} \mathrm{H}_{2} \mathrm{O}$ Band: Crystallization, Large Grains, and Hydrates . . . . . . . . . . . 11

The $4.67 \mu \mathrm{m} \mathrm{CO} \mathrm{Band:} \mathrm{the} \mathrm{Most} \mathrm{Volatile} \mathrm{Ices} \mathrm{.} \mathrm{.} \mathrm{.} \mathrm{.} \mathrm{.} \mathrm{.} \mathrm{.} \mathrm{.} \mathrm{.} \mathrm{.} \mathrm{.} \mathrm{.} \mathrm{.} \mathrm{.} \mathrm{.} \mathrm{.} \mathrm{.} \mathrm{.} \mathrm{.} \mathrm{.} \mathrm{.} 12$

The 15.2 $\mu \mathrm{m} \mathrm{CO} \mathrm{O}_{2}$ Band: Segregation and Distillation ................ 14 
The 6.0 and $6.85 \mu \mathrm{m}$ Features: Complexity Revealed . . . . . . . . . . . . . . 15

The Lattice Modes: Ice Structure and Emission . . . . . . . . . . . . . . . . . . . . . . . . 15

ICES ACROSS ENVIRONMENTS . . . . . . . . . . . . . . . . . . 16

Quiescent Dense Clouds and Cores . . . . . . . . . . . . . . . . . . 17

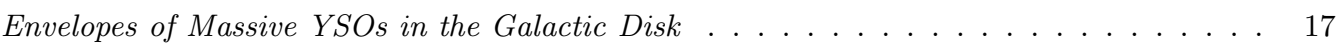

Envelopes of Low and Intermediate Mass YSOs . . . . . . . . . . . . . . . . . . . . . . . . 18

Disks Around Low and Intermediate Mass YSOs . . . . . . . . . . . . . . . . . . . . . . . 19

Galactic Center Region . . . . . . . . . . . . . . . . . . . . . . . 21

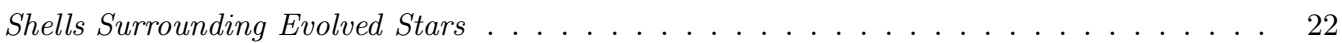

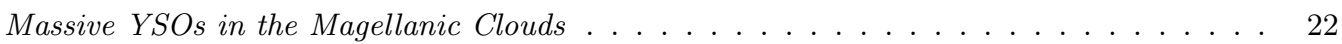

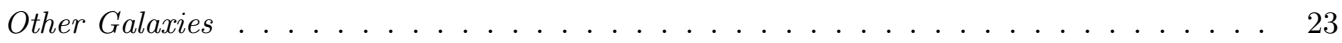

Solar System Objects . . . . . . . . . . . . . . . . . . . . 23

COLUMN DENSITIES AND ABUNDANCES . . . . . . . . . . . . . . . . 24

Identification Issues . . . . . . . . . . . . . . . . . . . . . . . . . . . . . . . 28

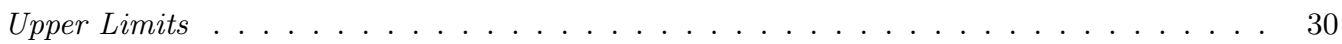

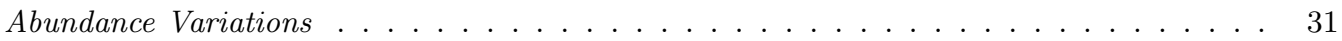

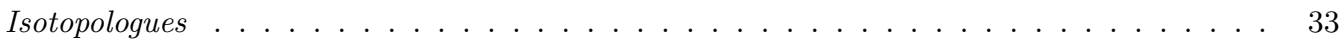

Interstellar Versus Cometary Ice Abundances . . . . . . . . . . . . . . . . . . . . 35

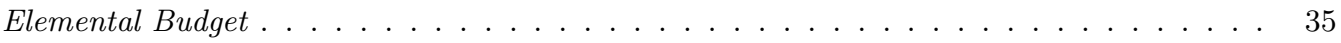

GRAIN SIZE AND MANTLE THICKNESS . . . . . . . . . . . . . . . 36

CONSTRAINTS ON ICE EVOLUTION MODELS . . . . . . . . . . . . . . . . . . . . . . . . . . . .

Accretion and Grain Surface Chemistry . . . . . . . . . . . . . . . . . . . . . . . . . . . . . . . . . . .

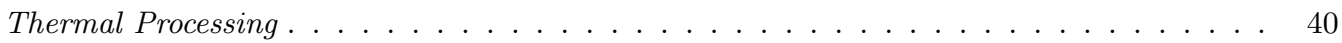

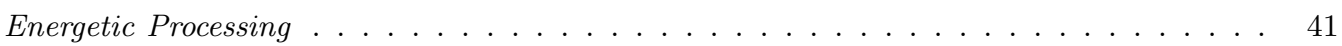

Connection with Cometary Ices . . . . . . . . . . . . . . . . . . . . . . 42

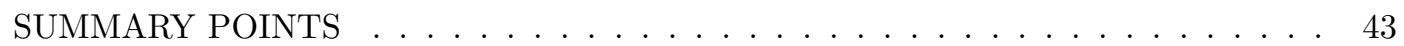

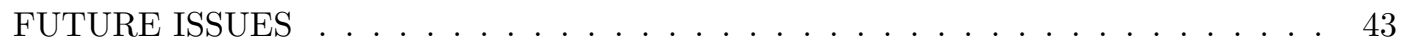

RELATED RESOURCES .......................... . . . . 44

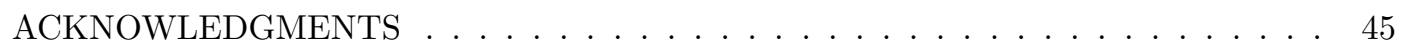

\section{PERSPECTIVE}

The condensation of gas phase elements on cold dust particles has long been considered an important process in the Universe. Lindblad (1935) studied the condensation of metals to form meteoritic material as the starting point of planet formation. Considering the elemental abundances, van de Hulst (1946) realized that the same pro-

(Interstellar) ice: solid state pure volatiles $\left(\mathrm{H}_{2} \mathrm{O}, \mathrm{CO}\right.$, etc.) or mixtures thereof, with, perhaps, refractory inclusions, located on interstellar dust grains. cess must lead to the formation of $\mathrm{H}_{2} \mathrm{O}, \mathrm{CH}_{4}$, and $\mathrm{NH}_{3}$ ices on the grains. Chemistry on cold grain surfaces has since emerged as a vital route to formation of new molecules in dense clouds and circumstellar envelopes and disks, including species that cannot readily be formed in the gas phase (Tielens \& Hagen 1982; Herbst \& van Dishoeck 2009 and references therein); surface chemistry thus controls the composition not only of the icy mantles but also of the molecular gas desorbed from the grains in the vicinity of cloud edges, shocks, and the radiation fields of Young Stellar Objects (YSOs).

Other chemical pathways to new interstellar ice species are observationally not as well established. Laboratory studies have shown that ice heating creates new 
molecules by purely thermal reactions (Schutte et al., 1993; Theulé et al., 2013) or through the increased mobility of radicals created by ultraviolet (UV) photons and cosmic ray (CR) particles. The latter has been a central topic in laboratory studies as a means to form complex molecules from simple ices. Heavy processing of ices forms organic residues (sometimes referred to as "yellow" and "brown stuff") that resemble the insoluble organic component of carbonaceous meteorites (Greenberg 1973; Greenberg et al. 1995, and references therein), and the products may include biologically relevant species (e.g., Bernstein et al. 1995; Muñoz Caro et al. 2004).

Ices are an important reservoir of the elements in dense $\left(>10^{3} \mathrm{~cm}^{-3}\right)$ environments; up to $60 \%$ of the oxygen not included in silicates is incorporated in known ice species. The processes that are key in their origin and evolution are thus directly relevant to key questions on the organic and volatile reservoir of regions of star and planet formation. What is the origin of the volatiles and organics of the solar nebula, possibly delivered to the early Earth as the building blocks of life? There is no clear consensus on whether these materials survived the voyage from circumstellar envelopes into protoplanetary disks (e.g., Visser et al. 2009; Brownlee 2014; Cleeves et al. 2014). Comets, believed to contain the most pristine remnant ices from the formation of the solar system (e.g., Mumma \& Charnley 2011), are key in answering this question.

In the broader astrophysical context, ices play important roles as well. For example, they speed up the grain coagulation process (Ormel et al., 2009). Also, questions on the importance of ice processing are directly related to questions about the origin, composition, and physical properties of interstellar dust. Energetically processed ices might be a source of refractory interstellar dust, in particular in star forming regions (Li et al. 2014 and references therein) as opposed to dust formed in the envelopes of evolved stars.

The initial searches for ices targeted the $3.0 \mu \mathrm{m} \mathrm{H}_{2} \mathrm{O}$ band in lines of sight dominated by diffuse dust (Danielson et al., 1965; Knacke et al., 1969). Gillett et al. (1975) noted that the lack of ices in these diffuse clouds could not be explained by elevated dust temperatures alone, revealing the importance of the non-thermal process of photodesorption (Watson \& Salpeter, 1972). The $3.0 \mu \mathrm{m}$ feature was finally detected toward the Orion BN/KL region (Gillett \& Forrest, 1973). Its position and width were in reasonable agreement with a model of small, pure $\mathrm{H}_{2} \mathrm{O}$ ice spheres, but significant deviations were observed as well (Merrill et al., 1976): short and long-wavelength wings were attributed to scattering by large grains and interactions with $\mathrm{NH}_{3}$ mixed in the ice (Merrill et al., 1976; Knacke et al., 1982; Smith et al., 1989). The latter supported the concept of ice mixtures (sometimes called "dirty ice") first proposed by van de Hulst (1946). Most ice features discovered thereafter show stunningly complex profiles as a result of the ice composition, structure (thermal history), and grain shape and size. For several bands (those of $\mathrm{H}_{2} \mathrm{O}, \mathrm{CO}$, and $\mathrm{CO}_{2}$ in particular) this is well understood, and their diagnostic value of the physical conditions and the nature of astronomical sources is a main topic of this review.

This is a review of observational studies of interstellar and circumstellar ices. The composition of the ices and evidence for formation and thermal and energetic modification processes are reviewed across Galactic and extragalactic environments. Previous reviews focused on massive YSOs (MYSOs hereafter; Gibb et al. 2004; Boogert \& Ehrenfreund 2004b; Dartois 2005) and on low mass YSOs (LYSOs hereafter) and background stars tracing nearby clouds (Öberg et al., 2011). In particular,

Energetic processing of ices: exposure to ionizing radiation, breaking bonds, and creating radicals and ions. Subsequent chemical reactions may lead to more complex molecules.

\section{Photodesorption:}

non-thermal desorption of a particle from a grain surface after a photon hit to itself or an underlying particle.

Dirty ice: illdefined term sometimes used to indicate that interstellar ices are mixtures, contain processed residues, or are present on grains. 
the study by Öberg et al. is expanded here to include the Galactic Center (GC) region and extragalactic environments, as well as to a full inventory of observed features and identifications. Solar system ices are not reviewed in detail, but the interstellar medium (ISM) and circumstellar ice properties are compared with those of comets as reviewed by Mumma \& Charnley (2011) to address the question of the origin of solar system volatiles and organics.

\section{ICES ACROSS THE ELECTROMAGNETIC SPECTRUM}

Ices in interstellar environments are traced almost exclusively by their molecular vibrational transitions in the near- to far-infrared. In general, the $1-3 \mu \mathrm{m}$ wavelength region contains the combination and overtone modes, the $3-6 \mu \mathrm{m}$ region the stretch vibrations, the $6-30 \mu \mathrm{m}$ region the bend and libration vibrations, and the 25-300 $\mu \mathrm{m}$ region the torsional and inter-molecular (lattice) modes. Some ices also have strong electronic transitions in the UV, e.g. in the case of $\mathrm{H}_{2} \mathrm{O}$ near $0.14 \mu \mathrm{m}$ (Warren \& Brandt, 2008). These UV features have not yet been observed in the ISM, due at least in part to very high UV extinction in candidate lines of sight. They may be detectable in absorption near cloud edges (at low $A_{\mathrm{V}}$ values; Goebel 1983) or through scattering. Finally, simple ices are transparent outside their vibrational modes, but the continuum opacities increase significantly for processed ices (Jenniskens, 1993; Brunetto et al., 2006) and ices with amorphous carbon inclusions (Preibisch et al., 1993).

The vast majority of interstellar and circumstellar ice studies target the stretch and bend mode vibrations in the $3-16 \mu \mathrm{m}$ range, because below $3 \mu \mathrm{m}$ the signals are weak as a result of dust continuum extinction (in contrast to solar system studies which often observe the combination modes at 1-2 $\mu \mathrm{m}$ ), and above $\sim 30 \mu \mathrm{m}$ the availability and capability of instrumentation are limited. Figures 1 and 2 show a selection of ice features detected outside the solar system and Table 1 gives a comprehensive summary of the typical peak positions, widths, and identifications. Nearly all features are securely detected, but not all are positively identified, as indicated with questions marks and further discussed in 6.1 . The widths vary dramatically, from $1.5 \mathrm{~cm}^{-1}(0.0034 \mu \mathrm{m})$ for ${ }^{13} \mathrm{CO}$ to $400 \mathrm{~cm}^{-1}(0.38 \mu \mathrm{m})$ for the $\mathrm{H}_{2} \mathrm{O}$ ice bands. Also, many band profiles (e.g., those of $\mathrm{CO}$ and $\mathrm{CO}_{2}$ ) contain substructures at the level of a few $\mathrm{cm}^{-1}$. Thus, ice studies benefit significantly from the availability of medium resolution infrared spectrometers $(R=\lambda / \Delta \lambda \geq 500)$. For many features, the positions and widths vary considerably across objects. These variations are often well studied, and have led to empirical decompositions. The components are listed, and are further discussed in 84 .

Indirect constraints on the ice composition are provided by observations of gasphase rotational lines at (sub)-millimeter wavelengths. First, low gas phase abundances (depletions) of specific molecules in clouds imply their presence in the ices (Bergin \& Tafalla, 2007). Second, gas phase species observed in hot core regions surrounding YSOs originate from sublimated ices, either directly or after gas phase chemical reactions (Blake et al., 1987; Herbst \& van Dishoeck, 2009). Third, some gas near cloud edges originates from photodesorbed ices (Öberg et al., 2009a). These results are not reviewed here, but are quoted when relevant. 


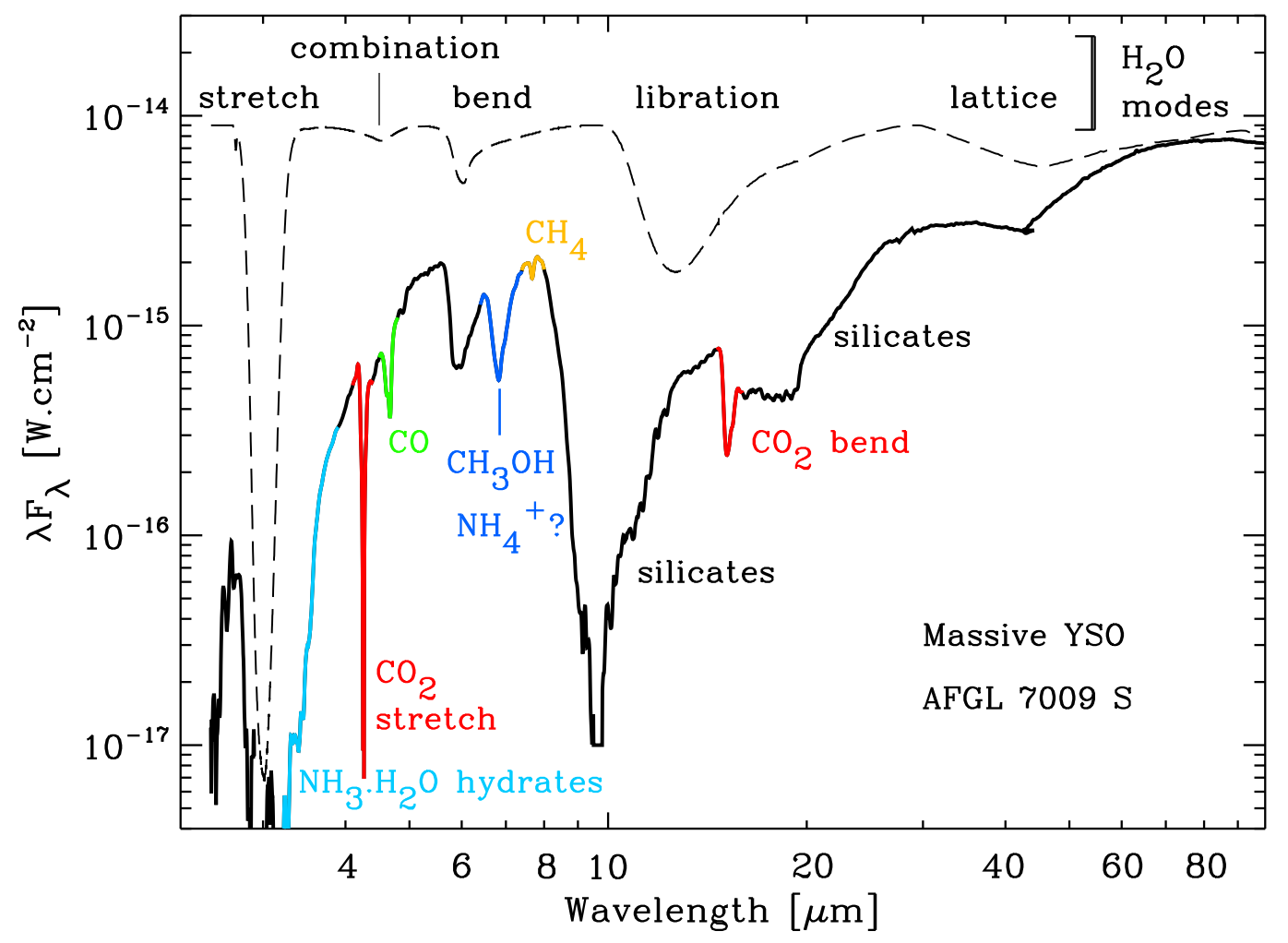

Figure 1: Overview of the strongest ice and dust features in the MYSO AFGL 7009S (Dartois et al., 1998). The calculated spectrum of pure $\mathrm{H}_{2} \mathrm{O}$ ice spheres at $10 \mathrm{~K}$ is shown (dashed line) to indicate the multiple $\mathrm{H}_{2} \mathrm{O}$ bands.

\subsection{Ice Mapping}

Most ice features are detected as pure absorption bands against infrared continuum point sources. Studies of the spatial variations of the ice properties are therefore relatively rare, yet very powerful. They often rely on the presence of many point sources (usually infrared-bright giants) behind clouds (e.g., Taurus; Murakawa et al. 2000). The envelope of a Class 0 YSO was mapped using background Class II LYSOs (Pontoppidan et al., 2004), and of a dense core using Class I/II LYSOs (Pontoppidan, 2006). Ice mapping is however also possible if the background emission is extended by scattering from dust in any disk, envelope, or outflow cone (Harker et al., 1997; Spoon et al., 2003; (Schegerer \& Wolf, 2010). In addition, scattering by large grains enhances the short-wavelength wing of the $3.0 \mu \mathrm{m} \mathrm{H}_{2} \mathrm{O}$ band 4.1 . Pendleton et al. 1990), which enables mapping of ices in reflection nebulae. This has rarely been done. Similarly, the lattice modes $(\sim 25-300 \mu \mathrm{m}$; 4.5$)$ are suited for ice mapping. They are the only ice bands that may appear in emission, because, in contrast to the intra-molecular modes, their excitation energy is below the binding energy of the ices. 


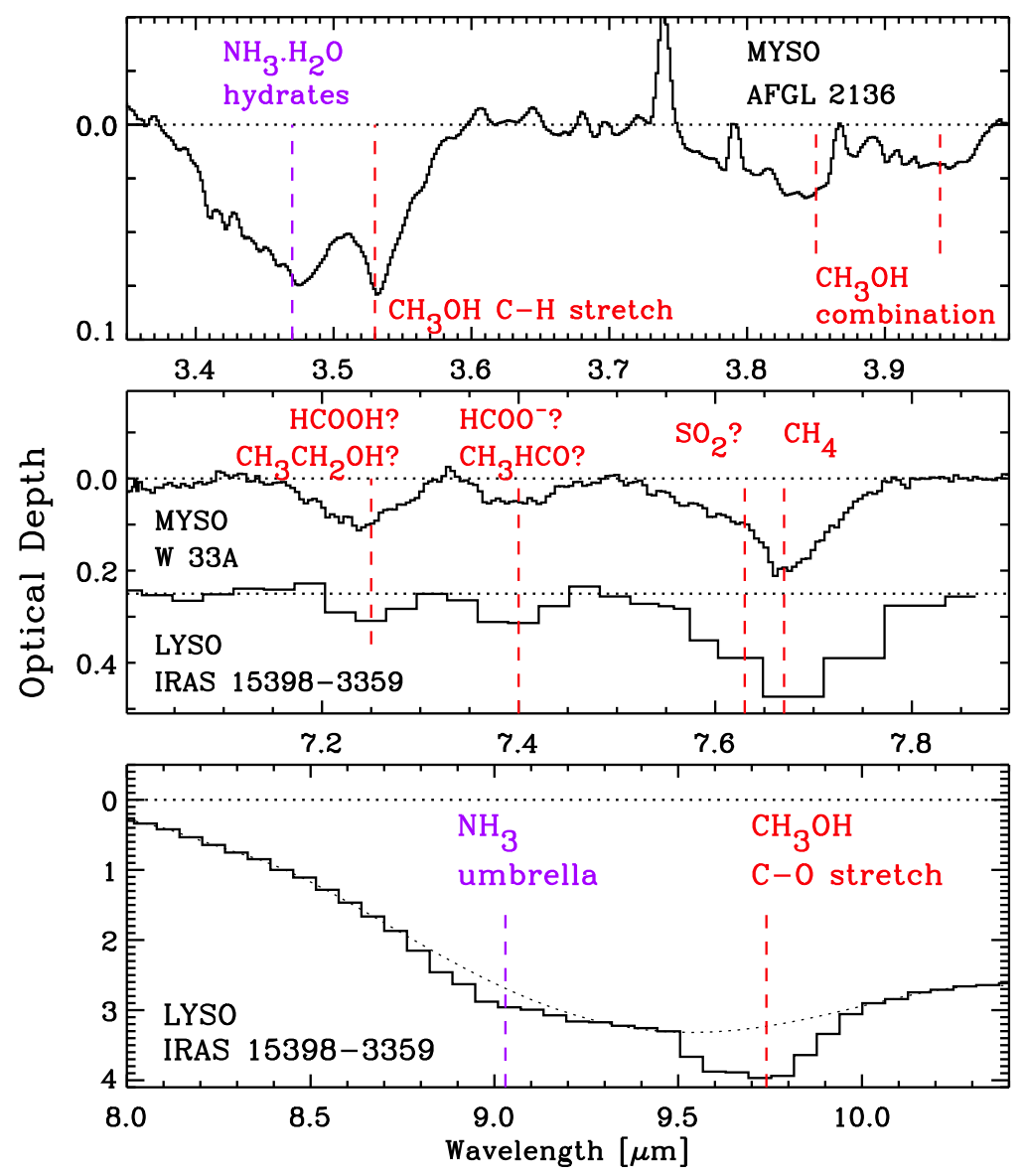

Figure 2: Compilation of weak ice absorption features in the 3.3-4.0 (top), 7.0-7.9 (middle) and 8.0-10.4 (bottom) $\mu \mathrm{m}$ regions. Identifications are labeled, and tentative identifications are followed by question marks. Data for AFGL 2136 were taken from Dartois et al. (2003), for W 33A from Schutte et al. (1999), and for IRAS 15398-3359 from Boogert et al. (2008) and Bottinelli et al. (2010). 
Table 1: Ice Features Detected in Interstellar and Circum-

stellar Environments

\begin{tabular}{|c|c|c|c|c|}
\hline $\begin{array}{l}\lambda_{\text {center }} \\
\mu \mathrm{m}\end{array}$ & $\begin{array}{l}\nu_{\text {center }} \\
\mathrm{cm}^{-1}\end{array}$ & $\begin{array}{c}\text { FWHM } \\
\mathrm{cm}^{-1}\end{array}$ & Identification & Reference $^{\mathrm{a}}$ \\
\hline 2.27 & 4405 & 60 & $\mathrm{CH}_{3} \mathrm{OH}$ combination & 1 \\
\hline 2.697 & 3708 & 4 & $\mathrm{CO}_{2}$ combination & 2 \\
\hline 2.778 & 3600 & 5 & $\mathrm{CO}_{2}$ combination & 2 \\
\hline 2.96 & 3378 & $\sim 40$ & $\mathrm{NH}_{3}$ stretch? & 3,4 \\
\hline 3.0 & 3333 & $\sim 390$ & $\mathrm{H}_{2} \mathrm{O}$ stretch (multiple components) ${ }^{\mathrm{b}}$ & \\
\hline-2.90 & 3448 & $\sim 120$ & — scattering & 5,6 \\
\hline-3.05 & 3278 & $\sim 315$ & — amorphous small grains & 7,8 \\
\hline-3.10 & 3225 & $\sim 220$ & — crystalline small grains & 8 \\
\hline$-\sim 3.3$ & 3030 & $\sim 270$ & — scattering and $\mathrm{NH}_{3} . \mathrm{H}_{2} \mathrm{O}$ hydrate & $9,10,16$ \\
\hline 3.25 & 3076 & 70 & PAH C-H stretch? $\mathrm{NH}_{4}^{+}$combination? & $11,12,13$ \\
\hline 3.325 & 3008 & 15 & $\mathrm{CH}_{4}$ stretch & 14 \\
\hline 3.47 & 2881 & 80 & $\mathrm{NH}_{3} \cdot \mathrm{H}_{2} \mathrm{O}$ hydrate & 15,4 \\
\hline 3.53 & 2832 & 30 & $\mathrm{CH}_{3} \mathrm{OH} \mathrm{C}-\mathrm{H}$ stretch & 17 \\
\hline 3.85 & 2597 & $\sim 40$ & $\mathrm{CH}_{3} \mathrm{OH}$ combination & 15 \\
\hline 3.94 & 2538 & $\sim 40$ & $\mathrm{CH}_{3} \mathrm{OH}$ combination & 18,15 \\
\hline $4.07^{\mathrm{c}}$ & 2457 & $\sim 120$ & HDO O-D stretch amorphous & 19 \\
\hline $4.13^{\mathrm{c}}$ & 2421 & $\sim 60$ & HDO O-D stretch crystalline & 19 \\
\hline 4.27 & 2341 & $\sim 20$ & $\mathrm{CO}_{2}$ stretch & 20 \\
\hline 4.39 & 2278 & 9 & ${ }^{13} \mathrm{CO}_{2}$ stretch (multiple components) & \\
\hline-4.382 & 2282 & 3 & - pure $\mathrm{CO}_{2}$ & 21 \\
\hline-4.390 & 2278 & 8 & - polar, $\mathrm{H}_{2} \mathrm{O} / \mathrm{CO}_{2}>1$ & 21 \\
\hline 4.5 & 2222 & 270 & $\mathrm{H}_{2} \mathrm{O}$ combination & 22 \\
\hline 4.62 & 2164 & 29 & "XCN" (multiple components) & 23 \\
\hline
\end{tabular}




\begin{tabular}{|c|c|c|c|c|}
\hline $\begin{array}{l}\lambda_{\text {center }} \\
\mu \mathrm{m}\end{array}$ & $\begin{array}{l}\nu_{\text {center }} \\
\mathrm{cm}^{-1}\end{array}$ & $\begin{array}{c}\text { FWHM } \\
\mathrm{cm}^{-1}\end{array}$ & Identification & Reference $^{\mathrm{a}}$ \\
\hline-4.598 & 2175 & 15 & $-\mathrm{OCN}^{-} \mathrm{C} \equiv \mathrm{N}$ stretch (apolar)? & 24,25 \\
\hline-4.617 & 2166 & 26 & $-\mathrm{OCN}^{-} \mathrm{C} \equiv \mathrm{N}$ stretch (polar) & 24,25 \\
\hline 4.67 & 2141.3 & $3-9$ & CO stretch (multiple components) & 26 \\
\hline-4.665 & 2143.7 & 3.0 & - apolar, $\mathrm{CO}_{2} / \mathrm{CO}>1 ?$ & 44,24 \\
\hline-4.673 & 2139.9 & 3.5 & — apolar, > 90\% CO & 23,24 \\
\hline-4.681 & 2136.5 & 10.6 & - polar, $\mathrm{CH}_{3} \mathrm{OH} / \mathrm{CO}>1$ & $23,24,27$ \\
\hline 4.779 & 2092.4 & 1.50 & ${ }^{13} \mathrm{CO}$ stretch (apolar) & 28 \\
\hline 4.90 & 2040 & 22 & OCS C-O stretch & 18,29 \\
\hline 5.83 & 1715 & 35 & $\mathrm{H}_{2} \mathrm{CO} \mathrm{C}-\mathrm{O}$ stretch & 30 \\
\hline 6.0 & 1666 & 160 & multiple components & 31 \\
\hline-6.0 & 1666 & $\sim 130$ & $-\mathrm{H}_{2} \mathrm{O}$ bend & 31 \\
\hline-5.84 & 1712 & 72 & - $\mathrm{HCOOH} \mathrm{C-O} \mathrm{stretch?}$ & $30,31,32$ \\
\hline-6.18 & 1618 & 111 & $-\mathrm{NH}_{3}$ bend, $\mathrm{H}_{2} \mathrm{O} / \mathrm{CO}_{2}=2, \mathrm{PAH}$ C-C stretch & 30,32 \\
\hline 6.0 & $\sim 1666$ & $\sim 330$ & organic residue or an-ions? & 33,32 \\
\hline 6.85 & 1459 & 85 & multiple components & 31 \\
\hline-6.755 & 1480 & 43 & $-\mathrm{CH}_{3} \mathrm{OH} \mathrm{C}-\mathrm{H}$ deformation, $\mathrm{NH}_{4}^{+}$bend? & 30,32 \\
\hline-6.943 & 1440 & 61 & $-\mathrm{NH}_{4}^{+}$bend? & 30,32 \\
\hline 7.24 & 1381 & 19 & $\mathrm{HCOOH}, \mathrm{CH}_{3} \mathrm{CH}_{2} \mathrm{OH}, \mathrm{C}-\mathrm{H}$ deformation? & 34 \\
\hline 7.41 & 1349 & 15 & $\mathrm{HCOO}^{-}, \mathrm{CH}_{3} \mathrm{CHO}, \mathrm{C}-\mathrm{H}$ deformation? & 34 \\
\hline 7.63 & 1310 & 28 & $\mathrm{SO}_{2}$ stretch? & 35 \\
\hline 7.674 & 1303 & 11 & $\mathrm{CH}_{4}$ deformation & 36,37 \\
\hline 8.865 & 1128 & 15 & $\mathrm{CH}_{3} \mathrm{OH} \mathrm{C}-\mathrm{H}_{3}$ rock & 38 \\
\hline 9.01 & 1109 & 50 & $\mathrm{NH}_{3}$ umbrella & 39 \\
\hline 9.74 & 1026 & 30 & $\mathrm{CH}_{3} \mathrm{OH} \mathrm{C-O}$ stretch & 38 \\
\hline 13.6 & 735 & $\sim 220$ & $\mathrm{H}_{2} \mathrm{O}$ libration & 40 \\
\hline
\end{tabular}




\begin{tabular}{lccll}
$\begin{array}{l}\lambda_{\text {center }} \\
\mu \mathrm{m}\end{array}$ & $\begin{array}{c}\nu_{\text {center }} \\
\mathrm{cm}^{-1}\end{array}$ & $\begin{array}{c}\text { FWHM } \\
\mathrm{cm}^{-1}\end{array}$ & Identification & Reference \\
\hline $15.1-15.3$ & $653-662$ & $16-28$ & $\mathrm{CO}_{2}$ bend $($ multiple components) & 20 \\
-15.31 & 653 & 22 & - polar, $\mathrm{CO}_{2} / \mathrm{H}_{2} \mathrm{O}<1$ & 24 \\
-15.10 & 662.2 & 11 & - apolar, $\mathrm{CO}_{2} / \mathrm{CO} \sim 1$ & 24 \\
-15.15 & 660.0 & 2.5 & - dilute, $\mathrm{CO}_{2} / \mathrm{CO}<<1$ & 24 \\
$-15.11+15.26$ & $655.3+661$ & 19 & - pure $\mathrm{CO}_{2}$ & 24 \\
-15.4 & 648 & 10 & - shoulder, $\mathrm{CH}_{3} \mathrm{OH} / \mathrm{CO}_{2}>>1$ & 24 \\
44 & 227 & $\sim 100$ & $\mathrm{H}_{2} \mathrm{O}$ lattice (amorphous) & $41,42,43$ \\
44 & 227 & $\sim 50$ & $\mathrm{H}_{2} \mathrm{O}$ lattice (crystalline) & $41,42,43$ \\
62 & 161 & $\sim 50$ & $\mathrm{H}_{2} \mathrm{O}$ lattice (crystalline) & $41,42,43$ \\
\hline
\end{tabular}

${ }^{a}$ reference to a publication contributing strongly to its identification or profile characterization: 1: Taban et al. 2003, 2: Keane et al. 2001a, 3: Chiar et al. 2000, 4: Dartois \& d'Hendecourt 2001, 5: Knacke et al. 1982, 6: Pendleton et al. 1990, 7: Gillett \& Forrest 1973, 8: Smith et al. 1989, 9: Merrill et al. 1976, 10: Knacke \& McCorkle 1987, 11: Sellgren et al. 1994, 12: Hardegree-Ullman et al. 2014, 13: Schutte \& Khanna 2003, 14: Boogert et al. 2004a, 15: Allamandola et al. 1992, 16: Dartois et al. 2002, 17: Grim et al. 1991, 18: Geballe et al. 1985, 19: Aikawa et al. 2012, 20: Gerakines et al. 1999, 21: Boogert et al. 2000a, 22: Boogert et al. 2000b, 23: Lacv et al. 1984, 24: Pontoppidan et al. 2003, 25: van Broekhuizen et al. 2005, 26: Soifer et al. 1979, 27: Cuppen et al. 2011, 28: Boogert et al. 2002a, 29: Palumbo et al. 1997, 30: Keane et al. 2001b, 31: Schutte et al. 1996, 32: Boogert et al. 2008, 33: Gibb \& Whittet 2002, 34: Schutte et al. 1999, 35: Boogert et al. 1997, 36: Lacv et al. 1991, 37: Boogert et al.|1996, 38: Skinner et al.|1992, 39: Lacv et al.|1998, 40: Cox 1989, 41: Omont et al. 1990, 42: Malfait et al. 1999, 43: Dartois et al. 1998, 44: Boogert et al. 2002b

${ }^{\mathrm{b}}$ Contributions from the $\mathrm{O}-\mathrm{H}$ stretch mode in $\mathrm{CH}_{3} \mathrm{OH}$ and perhaps $\mathrm{HCOOH}$ and other species are expected, but are not detected as separate features.

c Tentative detection. 


\section{LABORATORY EXPERIMENTS}

Laboratory experiments have been crucial in the identification of the observed interstellar ice features (Table 1), in understanding their profiles (\$4), and in determining the chemical origin and fate of their carriers ( $(\sqrt[8]{8})$. An overview of laboratory work is beyond the scope of this review, but key aspects will be emphasized here and mentioned throughout the remaining sections.

The peak positions, widths, and shapes of the observed ice bands are influenced by the composition and structure of the ices (crystallinity, density, and homogeneity) as a result of the attractive and repulsive dipole interactions between neighboring molecules. They are also influenced by the properties of the dust grains (size, shape, and the relative mantle and core volumes) and these effects can be calculated from the optical properties of the bulk materials. All of these influences must be taken into account in the proper assignment of carriers to the observed ice features and special care must be used in deriving physical and chemical information from the band profiles such as overall ice composition or temperature.

The absorption band profiles of ices and ice mixtures are routinely measured by laboratory transmission and reflection spectroscopy at high vacuum $\left(<10^{-7}\right.$ mbar) under temperature-controlled conditions (e.g., Hudgins et al. 1993). These laboratory samples may also be exposed to UV photons or to particles, simulating molecule formation and destruction by energetic processes in interstellar environments (e.g., Gerakines et al. 2001b). Optical constants are generally measured with a Kramers-Kronig analysis of a single spectrum using the ice's known thickness and visible refractive index. For $\mathrm{CO}$ and $\mathrm{CO}_{2}$, conflicting optical constants were reported (Ehrenfreund et al., 1997), and it was shown that the most accurate values are derived using polarized light at a range of sample thicknesses and incidence angles (Baratta \& Palumbo, 1998). Databases of laboratory transmission spectra and optical constants of interstellar ice analogs are listed in "Related Resources".

Infrared band strengths (\$6) can be determined directly from the ice's complex refractive index, or from laboratory spectra of an ice of a known thickness that must be determined independently. The band strengths of a particular molecule vary with ice temperature and overall ice composition, although measurements under astrophysical conditions are relatively scarce. The band strengths of $\mathrm{CO}$ and $\mathrm{CO}_{2}$ were shown to vary by less than $\sim 20 \%$ when measured at low temperatures, mixed together, or in an $\mathrm{H}_{2} \mathrm{O}$ ice matrix (Gerakines et al., 1995), but the strength of the $3.0 \mu \mathrm{m}$ $\mathrm{H}_{2} \mathrm{O}$ stretch mode is reduced by an order of magnitude when $\mathrm{H}_{2} \mathrm{O}$ is highly diluted (van Thiel et al., 1957; Ehrenfreund et al., 1996). Integrated strengths of common ice bands are listed in, e.g., Hudgins et al. (1993) and Gerakines et al. (1995).

Improvements in laboratory techniques have made it possible to measure fundamental parameters such as energy barriers, reaction rates, and photodesorption yields. We refer to Linnartz et al. (2011) for a recent review on this topic. Very briefly, this "bottom up" approach has been successful in creating $\mathrm{H}_{2} \mathrm{CO}$ and $\mathrm{CH}_{3} \mathrm{OH}$ (Watanabe \& Kouchi, 2002), $\mathrm{CO}_{2}$ (Ioppolo et al., 2011) and other species on cold surfaces with quantitative results. These have been used in grain surface-chemistry models and have successfully reproduced observed ice abundances under realistic astrophysical conditions (e.g., Cuppen et al. 2011). 


\section{ICE BAND PROFILES}

The profiles of the 3.0, 4.67, and $15.2 \mu \mathrm{m}$ ice features have proven to be sensitive probes of the physical conditions and the nature of a wide range of astronomical objects (\$5): dense clouds, Galactic and extragalactic YSOs, evolved stars, the Galactic Center region, and external galaxies. The lattice modes above $25 \mu \mathrm{m}$ show great promise as probes of the ice composition and processing history. The 6 and $6.8 \mu \mathrm{m}$ features, though not fully identified, were shown to have diagnostic value as well. All other bands are discussed in $\$ 6$.

\subsection{The $3.0 \mu \mathrm{m} \mathrm{H}_{2} \mathrm{O}$ Band: Crystallization, Large Grains, and $\mathrm{Hy}-$ drates}

The interstellar $3.0 \mu \mathrm{m}$ band (Fig. 3) is primarily due to the $\mathrm{O}-\mathrm{H}$ stretching mode of bulk $\mathrm{H}_{2} \mathrm{O}$ ice, because of the high abundance of this species and because of the very high intrinsic band strength induced by the hydrogen bonding network (van Thiel et al., 1957)). The bands of $\mathrm{H}_{2} \mathrm{O}$ ice mixed with apolar species peak below $3.0 \mu \mathrm{m}$, and, lacking the hydrogen bonding network, their intrinsic strengths are an order of magnitude smaller (Ehrenfreund et al., 1996). These features of isolated $\mathrm{H}_{2} \mathrm{O}$ have not been detected in interstellar environments.

The profile of the $3.0 \mu \mathrm{m}$ band is not fully explained by absorption by small pure $\mathrm{H}_{2} \mathrm{O}$ ice spheres (Merrill et al., 1976; Knacke \& McCorkle, 1987). Excess absorption is always present between 3.3 and $3.7 \mu \mathrm{m}$ (Fig. 3), and sometimes near $2.9 \mu \mathrm{m}$. The strengths of the long wavelength wing and the peak depth of the $3.0 \mu \mathrm{m}$ band correlate well. Some variations were reported (Pontoppidan et al., 2004; Thi et al., 2006; Noble et al., 2013), suggesting a dependence on $\mathrm{H}_{2} \mathrm{O}$ abundance, but this needs further work. In any case, Mie scattering calculations show that large grains $(\sim 0.5 \mu \mathrm{m}$; Smith et al. 1989; 97) might explain much but not all of the long wavelength wing. The remainder of the absorption is attributed to the $\mathrm{O}-\mathrm{H}$ stretching mode in ammonia hydrates (Knacke et al., 1982; Dartois \& d'Hendecourt, 2001). The distinct 3.47 $\mu \mathrm{m}$ feature is likely also a result of these hydrates (6.1, Dartois \& d'Hendecourt 2001).

The wing at $2.9 \mu \mathrm{m}$ is not as commonly observed. Its strength increases with the observational beam size, capturing larger contributions of light scattered off large ice-coated grains in some objects (e.g., the Orion BN/KL nebula in Pendleton et al. 1990). The absence of this feature in a background star and its presence towards YSOs and reflection nebulae further confirms this interpretation (Smith et al., 1989). The stretching mode of $\mathrm{NH}_{3}$ contributes to this wing, but it is rarely detected as a distinct feature (Chiar et al., 2000; Dartois \& d'Hendecourt, 2001).

The $3.0 \mu \mathrm{m}$ band profile varies considerably among targets within and across different environment classes (Fig. 3). The peak position and width toward the majority of observed YSOs and all background stars are consistent with amorphous $\mathrm{H}_{2} \mathrm{O}$ ice (Smith et al., 1989). Narrower profiles that are shifted to longer wavelengths toward some MYSOs and a few LYSOs (Boogert et al., 2008) reflect higher degrees of crystallinity attributed to thermal processing (88.2). An extreme case is that of the edge-on low mass disk source d216-0939 (Terada \& Tokunaga, 2012a), which shows a peak at $\sim 3.20 \mu \mathrm{m}$ caused by large $(\sim 0.8 \mu \mathrm{m})$ grains consisting of nearly pure crystalline $\mathrm{H}_{2} \mathrm{O}$ ice.
Hydrogen bonding network: dipole attraction between the electropositive $\mathrm{H}$ from one molecule with the electronegative atom (e.g., $\mathrm{O}$ or $\mathrm{N}$ ) from another.

Ammonia hydrates: a hydrogen bonding network between $\mathrm{H}_{2} \mathrm{O}$ and $\mathrm{NH}_{3}$, often noted as $\mathrm{H}_{2} \mathrm{O} \cdot \mathrm{NH}_{3}$.

Thermal processing of ices: increase in ice temperature, leading to sublimation, a rearrangement of molecular bonds (crystallization and segregation), or chemical reactions. 

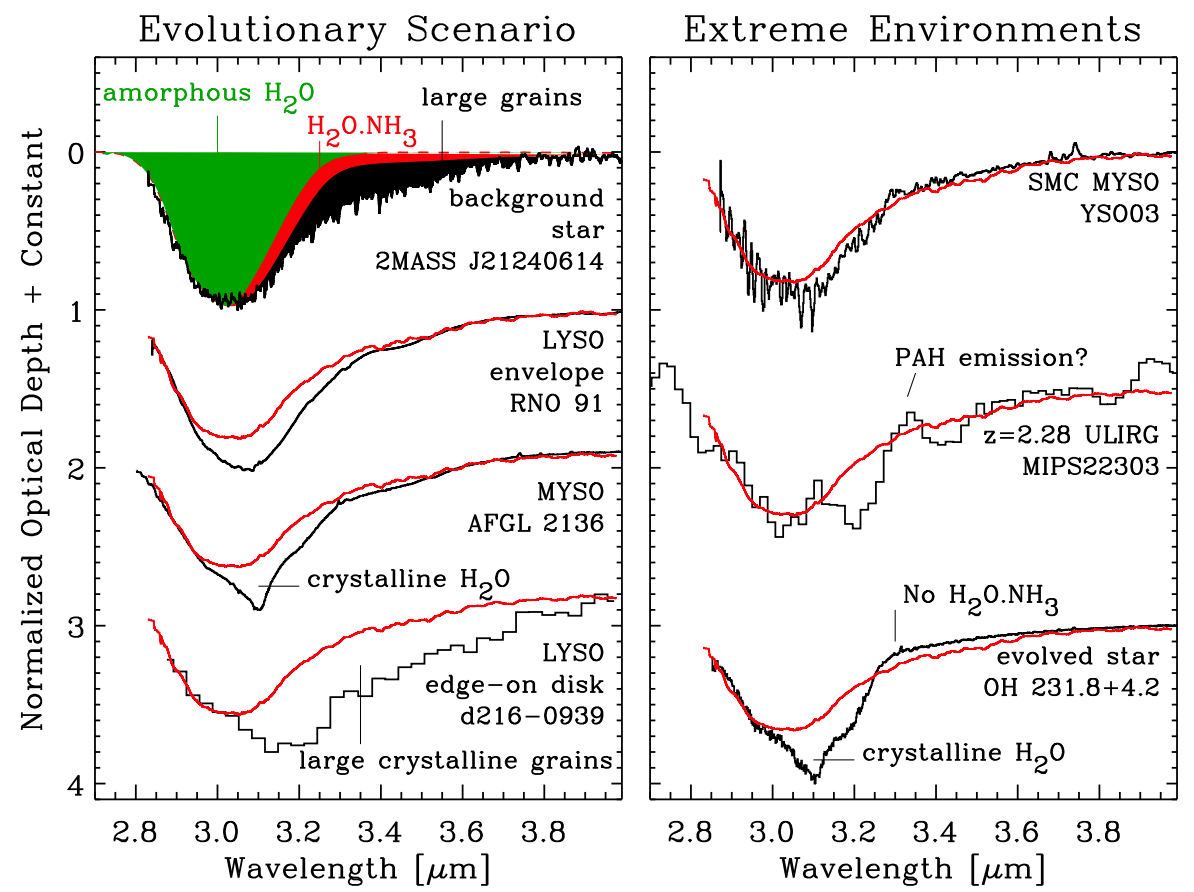

Figure 3: Observed $3.0 \mu \mathrm{m}$ band profiles, highlighting the universally present $\mathrm{H}_{2} \mathrm{O}$ ice components and their variations. The left panel shows sightlines with, from top to bottom, increasing degrees of crystallinity as a result of heating above temperatures of $\sim 70 \mathrm{~K}$ : the background star 2MASSJ 21240614+4958310 (Boogert et al., 2011), the LYSO RNO 91 (Boogert et al., 2008), the Galactic MYSO GL2136 (Dartois et al., 2002), and the edge-on disk source d216-0939 (Terada \& Tokunaga, 2012a). The right panel shows the SMC MYSO 03 (Oliveira et al., 2013), the ULIRG galaxy MIPS 22303 at $\mathrm{z}=2.28$ (Sajina et al. 2009), and the evolved star $\mathrm{OH} 231.8+4.2$ (Dartois et al., 2002). For comparison, the smoothed spectrum of the background star is plotted over each spectrum (red). The background star itself is compared with pure, amorphous $\mathrm{H}_{2} \mathrm{O}$ ice particles (CDE shapes) at $10 \mathrm{~K}$ (green area). If instead a $\mathrm{H}_{2} \mathrm{O}: \mathrm{NH}_{3}=10: 1$ spectrum is used, part of the long-wavelength wing is explained by $\mathrm{NH}_{3}$ hydrates $\left(\mathrm{NH}_{3} \cdot \mathrm{H}_{2} \mathrm{O}\right.$; red area). The black area is the residual due to large $(\sim 0.35 \mu \mathrm{m})$ grains.

\subsection{The 4.67 $\mu \mathrm{m}$ CO Band: the Most Volatile Ices}

The $4.67 \mu \mathrm{m}$ band consists of three components as a result of solid $\mathrm{CO}$ in different molecular environments. Gaussian representations of these components fit all observed CO ice bands toward YSOs and background stars (Table 1) Fig. 4), and their relative strengths may be used to derive the ice composition and dust temperature

Polar ices: ices dominated by molecules with high dipole moments (e.g., $\mathrm{H}_{2} \mathrm{O}, \mathrm{CH}_{3} \mathrm{OH}$ ), which have relatively high sublimation temperatures. along the line of sight (Pontoppidan et al., 2003). A narrow feature centered on $4.673 \mu \mathrm{m}$ and a much broader long wavelength wing peaking at $4.681 \mu \mathrm{m}$ are traditionally attributed to $\mathrm{CO}$ embedded in low (apolar) and high (polar) dipole moment species (Sandford et al., 1988). The sublimation temperature of the polar component is $\sim 90 \mathrm{~K}$, much higher than that of the apolar component $(\sim 20 \mathrm{~K})$, and thus the ratio of their column densities is often used to trace the ice temperature (Fig. 4: Tielens et al. 1991; Chiar et al. 1998). For the polar CO component, entrapment or migration of $\mathrm{CO}$ into the abundantly present $\mathrm{H}_{2} \mathrm{O}$ ice seems an evident explanation, but the expected band at $4.647 \mu \mathrm{m}\left(2152 \mathrm{~cm}^{-1}\right)$ due to dangling $\mathrm{OH}$ bonds 


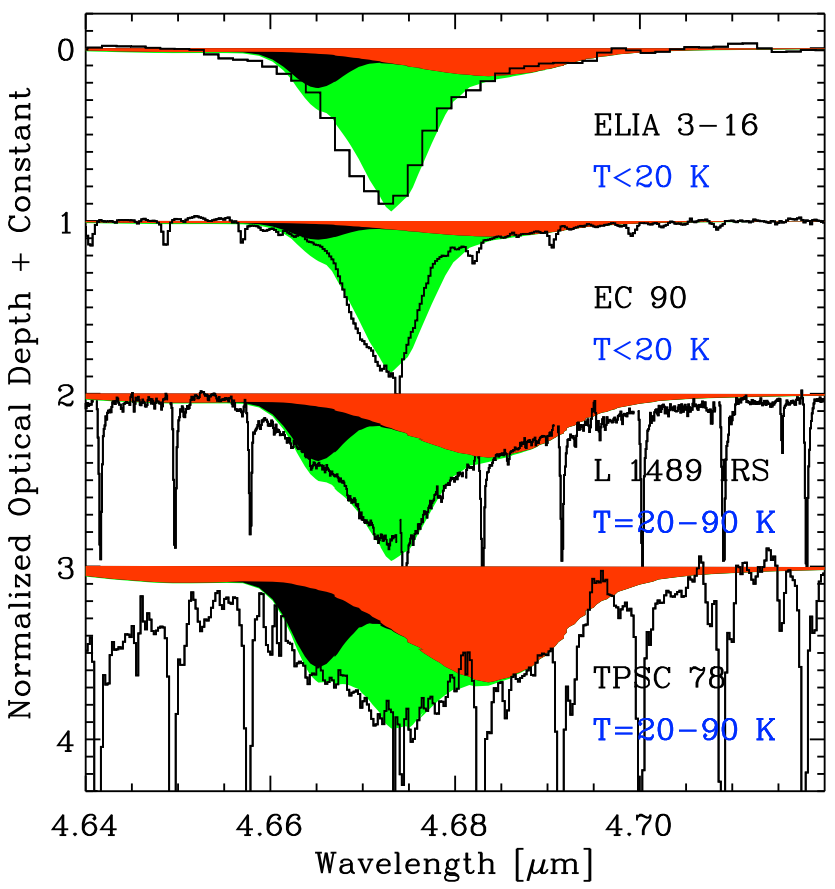

Figure 4: The CO ice band profile varies strongly with line of sight temperature. The Taurus background star Elias 16 (Chiar et al., 1995) and the LYSO EC 90 (Pontoppidan et al., 2003) trace dust below the pure CO sublimation temperature of $\sim 20 \mathrm{~K}$, while the LYSO L 1489 IRS (Boogert et al., 2002b) and the intermediate mass YSO TPSC 78 (Pontoppidan et al., 2003) have temperatures primarily above that. Two laboratory spectra are used to fit the profiles: pure CO corrected for CDE grain shapes (green) and $\mathrm{CO}$ in polar ices (red). The polar ice is $\mathrm{H}_{2} \mathrm{O}: \mathrm{CO}=4: 1$ in this case, but mixtures with $\mathrm{CH}_{3} \mathrm{OH}$ are likely more appropriate $(\$ 4.2)$. The blackcolored feature on the short-wavelength side is a Gaussian representing a CO ice that probably contains $\mathrm{CO}_{2}$, for lack of appropriate laboratory spectra. Ice sublimation is also evident by the increasing depth of the narrow ro-vibrational lines from gas phase CO.

in interstellar spectra is missing (Sandford et al., 1988). Mixtures with $\mathrm{CH}_{3} \mathrm{OH}-$ rich ices are more likely, both spectroscopically and chemically ( 88.1 , Cuppen et al. 2011). High spectral resolution observations have resolved the apolar component into a blue-shifted shoulder at $4.665 \mu \mathrm{m}$ and a very narrow feature at $4.673 \mu \mathrm{m}$ (Boogert et al., 2002b). The latter traces nearly $(\geq 90 \%)$ pure CO. The detection of the solid ${ }^{13} \mathrm{CO}$ band at $4.78 \mu \mathrm{m}$ was pivotal in this assessment (Boogert et al., 2002a). The absorption profile of this trace species is insensitive to particle shape effects, and very sensitive to the ice composition. For the ${ }^{12} \mathrm{CO}$ band, grains shaped according to the Continuous Distribution of Ellipsoids (CDE) in the Rayleigh limit provide the best fit. The composition of the $4.665 \mu \mathrm{m}$ component is less clear. It most likely contains $\mathrm{CO}_{2}$, supported by a good correlation of its strength with that of the $\mathrm{CO}: \mathrm{CO}_{2}$ component in the $15.2 \mu \mathrm{m} \mathrm{CO}_{2}$ bending mode (Pontoppidan et al., 2008), but a comparison with laboratory spectra shows that additional species are required in the mixture as well (Boogert et al., 2002b). An alternative explanation by absorption of linearly polarized light in a crystalline $\mathrm{CO}$ ice phase (the longitudinal optical mode; Pontoppidan et al. 2003) appears less likely.

Apolar ices: ices dominated by molecules with low dipole moments (e.g., $\mathrm{CO}, \quad \mathrm{N}_{2}$ ), which have relatively low sublimation temperatures. 
Particle shape effects: band profile changes caused by resonances between the molecular dipoles and the particle charge distribution induced by the radiation field (Bohren \& Huffman, 1983).

Ice distillation: sublimation of the most volatile species in mixtures (e.g., $\mathrm{CO}$ in $\mathrm{CO}_{2}$ ice), leaving the species with higher sublimation temperatures behind.

\subsection{The $15.2 \mu \mathrm{m} \mathrm{CO}$ Band: Segregation and Distillation}

The $15.2 \mu \mathrm{m}$ band of $\mathrm{CO}_{2}$ has proven to be a particularly powerful tracer of the composition and processing history of the ices (Fig. 51). Building on initial work on ISO observations of MYSOs by Gerakines et al. (1999), Pontoppidan et al. (2008) use the much larger sample observed by Spitzer toward LYSOs for an empirical decomposition of the complex band profiles. All observed bands can be fitted with a combination of 5 laboratory spectra, that are fixed in position and shape (Table 1), but whose relative abundances vary as a result of the conditions along the line of sight (Fig. 5): $\mathrm{CO}_{2}$ ("pure"), $\mathrm{CO}_{2} / \mathrm{CO} \sim 1$ ("apolar"), $\mathrm{CO}_{2} / \mathrm{CO}<<1$ ("dilute"), $\mathrm{CO}_{2} / \mathrm{H}_{2} \mathrm{O}<1$ ("polar"), and $\mathrm{CO}_{2} / \mathrm{CH}_{3} \mathrm{OH}<1$ ("shoulder"). The dominance of the polar component is strongly supported by the correlation of the $\mathrm{CO}_{2}$ and $\mathrm{H}_{2} \mathrm{O}$ column densities (\$6.3). A subset of YSOs shows distinct peaks at 15.11 and 15.26 $\mu \mathrm{m}$, which are attributed to those of pure $\mathrm{CO}_{2}$ (the symmetry of this linear molecule is broken in the pure ice matrix). These are the YSOs with the warmer envelopes, and it is thus concluded that pure $\mathrm{CO}_{2}$ ice is formed by the sublimation of $\mathrm{CO}$ from apolar ices ("distillation"; $20 \mathrm{~K}$ ) and at higher temperatures by "segregation" from polar ices as the $\mathrm{H}_{2} \mathrm{O}$ crystallizes and the rearrangement of bonds leads to clusters of pure $\mathrm{CO}_{2}$ (Ehrenfreund et al. 1999; 30-77 K). A few sources show a third peak at $15.35 \mu \mathrm{m}$, which is attributed to bonding of the carbon atoms of $\mathrm{CH}_{3} \mathrm{OH}$ with
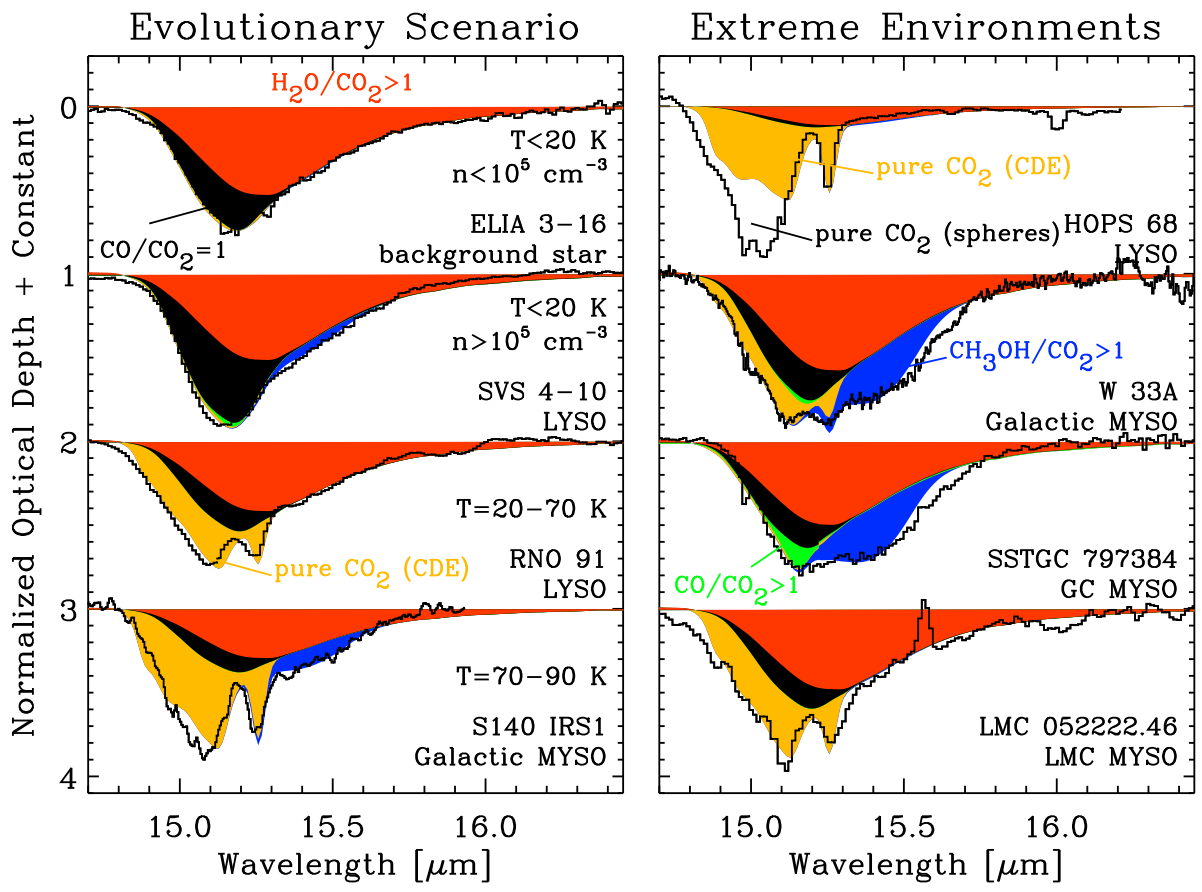

Figure 5: $\mathrm{CO}_{2}$ bending mode profiles observed in a range of environments. The colored areas show the components (\$4.3): polar (red), apolar (black), dilute (green), pure (yellow), and shoulder (blue). The left panel highlights an evolutionary scenario, showing, from top to bottom, the effects of increasing density and subsequent heating. The right panel shows sources that have experienced an extreme heating event (HOPS 68), have a high $\mathrm{CH}_{3} \mathrm{OH}$ abundance (W 33A), or high (SST GC 797384) and low (LMC 052222.46) metallicities. The data were taken from Gerakines et al. (1999), Bergin et al. (2005), Pontoppidan et al. (2008), An et al. (2011), Seale et al. (2011), and Poteet et al. (2013). 
the oxygen atoms of $\mathrm{CO}_{2}$ (the "shoulder" component). This is not an indicator of thermal processing (Dartois et al., 1999). Finally, the "apolar" component is probably responsible for the $4.66 \mu \mathrm{m}$ component of the $\mathrm{CO}$ band $(4.2)$ as well, and indeed their strengths correlate (Pontoppidan et al., 2008).

The profiles of the $\mathrm{CO}_{2}$-rich components ("apolar" and "pure") of the strong $15.2 \mu \mathrm{m}$ band are affected by grain shapes in addition to composition and thermal history. As for CO, good fits are obtained by CDE shaped grains in the Rayleigh limit. The essential confirmation comes from observations of the ${ }^{13} \mathrm{CO}_{2}$ band at $4.38 \mu \mathrm{m}$ (Boogert et al., 2000a), which is insensitive to the grain shapes and can be fitted with the same $\mathrm{CO}_{2}$ components. One striking exception of the CDE model is the LYSO HOPS 68, which shows convincing evidence for a population of spherical grains, reflecting an unusual history of sublimation and rapid re-condensation (Fig. 5 . Poteet et al. 2013).

\subsection{The 6.0 and 6.85 $\mu \mathrm{m}$ Features: Complexity Revealed}

The bending mode of $\mathrm{H}_{2} \mathrm{O}$ peaks at $6.0 \mu \mathrm{m}$ and overlaps with an $\mathrm{H}_{2} \mathrm{O}$ combination mode that extends to $8 \mu \mathrm{m}$. These are much weaker than the $3.0 \mu \mathrm{m}$ stretch mode (4.1), because they benefit less from the hydrogen bonding network (van Thiel et al., 1957). $\mathrm{H}_{2} \mathrm{O}$ absorption being less dominant, the 5-7 $\mu \mathrm{m}$ region has proven to be a good tracer of the complex composition of the ices (Fig. 6), despite considerable identification issues (\$6.1).

High spectral resolution observations of MYSOs with ISO revealed variations of the peak position of the $6.85 \mu \mathrm{m}$ feature (Keane et al., 2001b), which could be attributed to the thermal history. Also, a prominent broad absorption overlying both the 6.0 and $6.85 \mu \mathrm{m}$ features was discovered in a few sources (Gibb \& Whittet, 2002; Gibb et al., 2004). The 5-7 $\mu \mathrm{m}$ absorption observed toward LYSOs and background stars with Spitzer was decomposed in five components, after the subtraction of pure $\mathrm{H}_{2} \mathrm{O}$ ice (Fig. 6, Boogert et al. 2008), essentially confirming the earlier results toward MYSOs: C1 $(5.84 \mu \mathrm{m})$ and C2 $(6.18 \mu \mathrm{m})$, C3 $(6.755 \mu \mathrm{m})$ and C4 $(6.943 \mu \mathrm{m})$ and component C5 peaking at $5.9 \mu \mathrm{m}$ but stretching over the full $5.8-8 \mu \mathrm{m}$ range. Each component probably represents more than one carrier. The $\mathrm{C} 1$ and $\mathrm{C} 3$ components vary little relative to the $\mathrm{H}_{2} \mathrm{O}$ column density. Component $\mathrm{C} 4$ and perhaps $\mathrm{C} 2$ become more prominent at low $\mathrm{H}_{2} \mathrm{O}$ ice abundances, i.e., at temperatures above $\mathrm{H}_{2} \mathrm{O}$ sublimation $(>90 \mathrm{~K})$. These features can thus be treated as tracers of extreme conditions. Such cases have proven to be rare for LYSOs. Enhancements of the C5 component do not appear to correlate with those of $\mathrm{C} 4$, and may thus trace different processing conditions (e.g., at lower temperatures or by energetic photons or particles). Measurements of the strength and shape of this component are inaccurate, however, because it is so wide and overlaps with the $\mathrm{H}_{2} \mathrm{O}$ bending mode (Fig. 6).

\subsection{The Lattice Modes: Ice Structure and Emission}

Although the lattice modes were detected early on (in Orion-KL; Erickson et al. 1981), relatively few observations were published due to the limited availability of telescope instrumentation at wavelengths above $25 \mu \mathrm{m}$. These observations do show unequivocally the sensitivity of the modes to the thermal history of the ice (Malfait et al., 1999; Dartois et al., 1998; Omont et al., 1990; Sylvester et al., 1999; Demvk et al., 2000; Chiang et al., 2001). The $\mathrm{H}_{2} \mathrm{O}$ feature at $62 \mu \mathrm{m}$ is particularly

Spitzer: Spitzer Space Telescope, operating the IRS spectrometer (2003$2009)$ at $R \sim 60-128$ $(5-14 \quad \mu \mathrm{m}) \quad$ and $\sim 100-600(10-37$

$\mu \mathrm{m})$.

ISO: Infrared Space Observatory (19951998), operating spectrometers at wavelengths of 2.3-200 $\mu \mathrm{m}$ and resolving powers of up to $R=\lambda / \Delta \lambda \sim 2000$. 

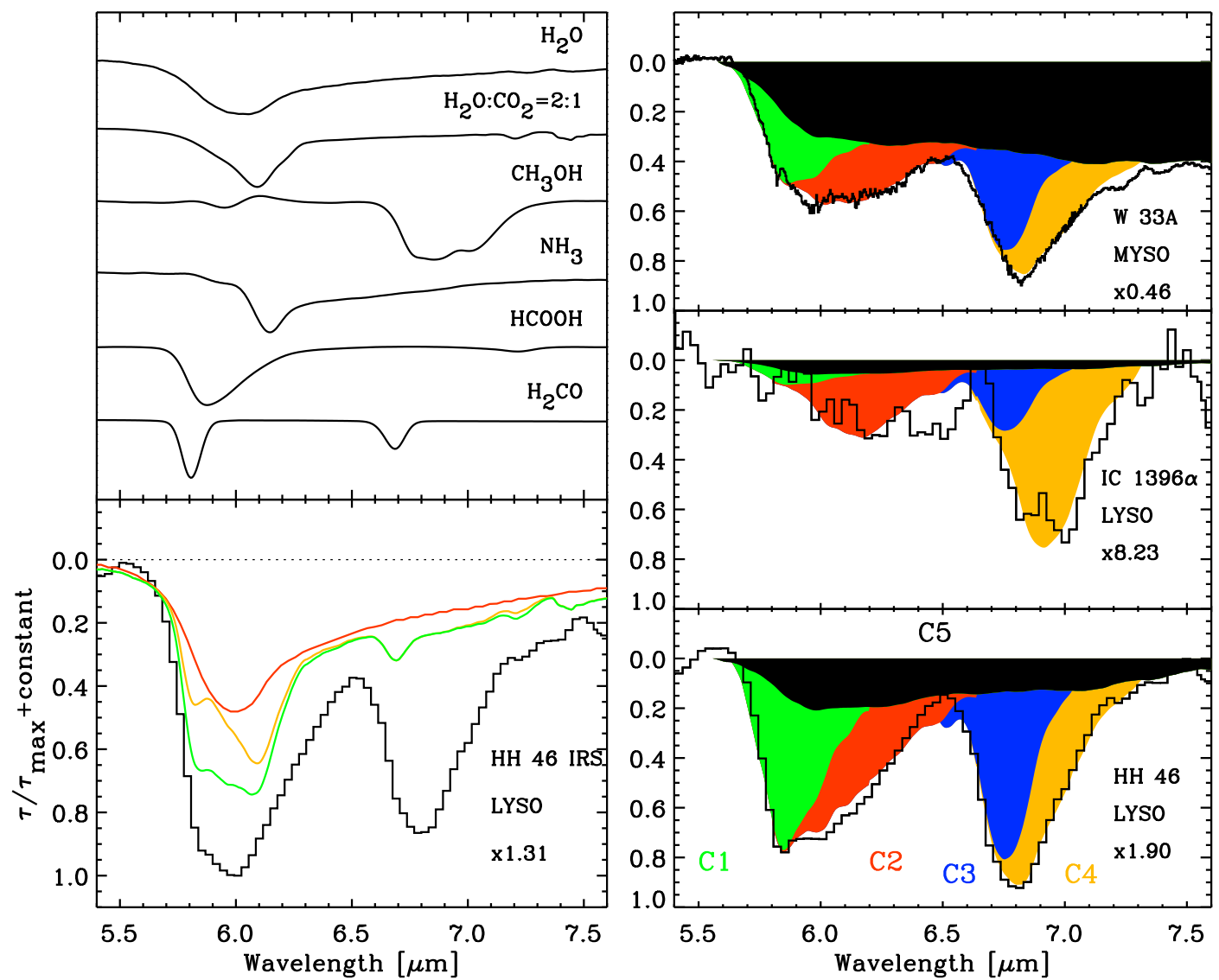

Figure 6: Left Panels: Laboratory spectra of species with modes in the 5-8 $\mu \mathrm{m}$ region. The sum of these is compared to the observed 5-8 $\mu \mathrm{m}$ features toward the LYSO HH 46 IRS (Boogert et al., 2008). The pure $\mathrm{H}_{2} \mathrm{O}$ contribution is given in red and the sum of all laboratory spectra including and excluding the uncertain $\mathrm{HCOOH}$ identification in green and yellow, respectively. A significant amount of absorption is unaccounted for. Right Panels: Example of the decomposition of the $\mathrm{H}_{2} \mathrm{O}$ subtracted spectra of the MYSO W 33A (Keane et al., 2001b), the LYSO IC 1396 $\alpha$ (Reach et al., 2009), and the LYSO HH 46 in five empirically determined components C1-C5, used to investigate source-to-source variations of the band profiles.

strong in crystalline ices. Laboratory experiments have shown that the bands are very sensitive to the ice composition as well, because impurities affect the long range modes (Moore \& Hudson, 1994; Ioppolo et al., 2014). In fact, along with the torsional modes at similar wavelengths, they identify complex species more uniquely than do the mid-infrared intra-molecular vibrations. Finally, as the lattice modes may appear in emission, they show great promise as tools to map the ice properties.

\section{ICES ACROSS ENVIRONMENTS}

Ices are present in a wide range of environments outside of the solar system: envelopes and disks of young and evolved stars and Galactic and extragalactic molecular clouds and cores (Fig. 3). These environments are characterized by high densities $\left(\geq 10^{3} \mathrm{~cm}^{-3}\right)$, low temperatures $(<90 \mathrm{~K})$ and weak UV radiation fields $\left(G_{0} \leq 0.07\right.$ at $A_{\mathrm{V}} \geq 1.6 \mathrm{mag} ; 8.3$ Hollenbach et al. 2009). The same species are generally 
detected in all environments, with the exception of the envelopes of evolved stars which only show $\mathrm{H}_{2} \mathrm{O}$ ice. The ice abundances vary greatly, however, both across and within environment classes. This reflects differences in physical conditions, age, and elemental abundances. Observational studies of the different environments are briefly reviewed here, and abundances are summarized in 96 ,

\subsection{Quiescent Dense Clouds and Cores}

The first detections of the $3.0 \mu \mathrm{m} \mathrm{H}_{2} \mathrm{O}$ ice band toward background stars showed that a dense cloud or core environment and a threshold extinction are needed for the formation of detectable quantities of $\mathrm{H}_{2} \mathrm{O}$ ices (Whittet et al., 1983). In Taurus, the ice band is only present at extinctions above $A_{\mathrm{V}}=3.2 \pm 0.1 \mathrm{mag}$ (Whittet et al., 2001), which corresponds to an ice formation threshold of $A_{\mathrm{V}}=1.6$ mag considering that background stars trace both sides of the cloud. Similar values are seen (Fig. 7) in the Lupus cloud (Boogert et al., 2013) as well as the dense cores L183 (Whittet et al., 2013) and IC5146 (Chiar et al., 2011). Larger thresholds claimed for some environments are not as well established. Toward the Oph cloud, generally considered a high radiation environment hindering ice growth, few background stars were observed and threshold determinations include YSOs (Tanaka et al., 1990).

Solid $\mathrm{CO}_{2}$, which was found to be ubiquitous in quiescent dense clouds (Whittet et al., 1998), has effectively the same formation threshold as $\mathrm{H}_{2} \mathrm{O}$ (Bergin et al., 2005; Whittet et al., 2009), showing a close chemical connection between these species. Still, their relative abundances vary by up to a factor of three (\$6.3). Freeze-out of the more volatile CO species occurs deeper into the cloud, at $\sim 3$ mag (Taurus, Serpens; Fig. 7, Chiar et al. 1995). Kerr et al. (1993) determine a three times larger threshold in Ophiuchus, but along individual lines of sight, values are lower, probably due to clumpiness (Shuping et al., 2000). $\mathrm{CH}_{3} \mathrm{OH}$ ice forms at even greater cloud depths (9 \pm 3 mag; Boogert et al. 2011; Chiar et al. 2011; Whittet et al. 2011b), consistent with the formation requirement of "catastrophic" CO freeze out (\$8.1). This is reflected in large $\mathrm{CH}_{3} \mathrm{OH}$ abundance variations, from less than $3 \%$ in Taurus (Chiar et al., 1996) to $12 \%$ in some isolated dense cores (Boogert et al., 2011; Chiar et al., 2011).

A general characteristic is the pristine nature of the ices in these environments: the dominance of amorphous $\mathrm{H}_{2} \mathrm{O}$ (Smith et al., 1993), the large contribution of apolar ices to the CO band (Whittet et al., 1985), and the absence of segregated (heated) $\mathrm{CO}_{2}$ ices (Whittet et al., 2009). The peak position of the $6.85 \mu \mathrm{m}$ feature is also consistent with unprocessed ices (Knez et al., 2005; Boogert et al., 2011). The absence of the $4.62 \mu \mathrm{m}$ "XCN" feature (Whittet et al., 1985; N Noble et al., 2013) is sometimes interpreted as a lack of processing, but, more likely, it is related to insufficient CO freeze-out ( $\$ 6.1$ and 98 ).

\subsection{Envelopes of Massive YSOs in the Galactic Disk}

A sample of $\sim 20$ MYSOs (luminosities above $10^{4} \mathrm{~L}_{\odot}$, masses above $10 \mathrm{M}_{\odot}$ ) in the Galactic Disk has been studied extensively in the ice features, using mostly data from ISO. We refer to reviews by Gibb et al. (2004), Boogert \& Ehrenfreund (2004b), and Dartois (2005) for historical work. Few new observations of these objects have been

Ice formation threshold: cloud extinction depth $\left(A_{\mathrm{V}}\right)$ indicating the onset of rapid ice mantle growth, after a monolayer has formed.

Monolayer: one layer of molecules covering dust grain surfaces, for $\mathrm{H}_{2} \mathrm{O}$ corresponding to $10^{15}$ molecules $/ \mathrm{cm}^{2}$ and an abundance $X_{\mathrm{H}} \sim$ few $\times 10^{-6}$. 


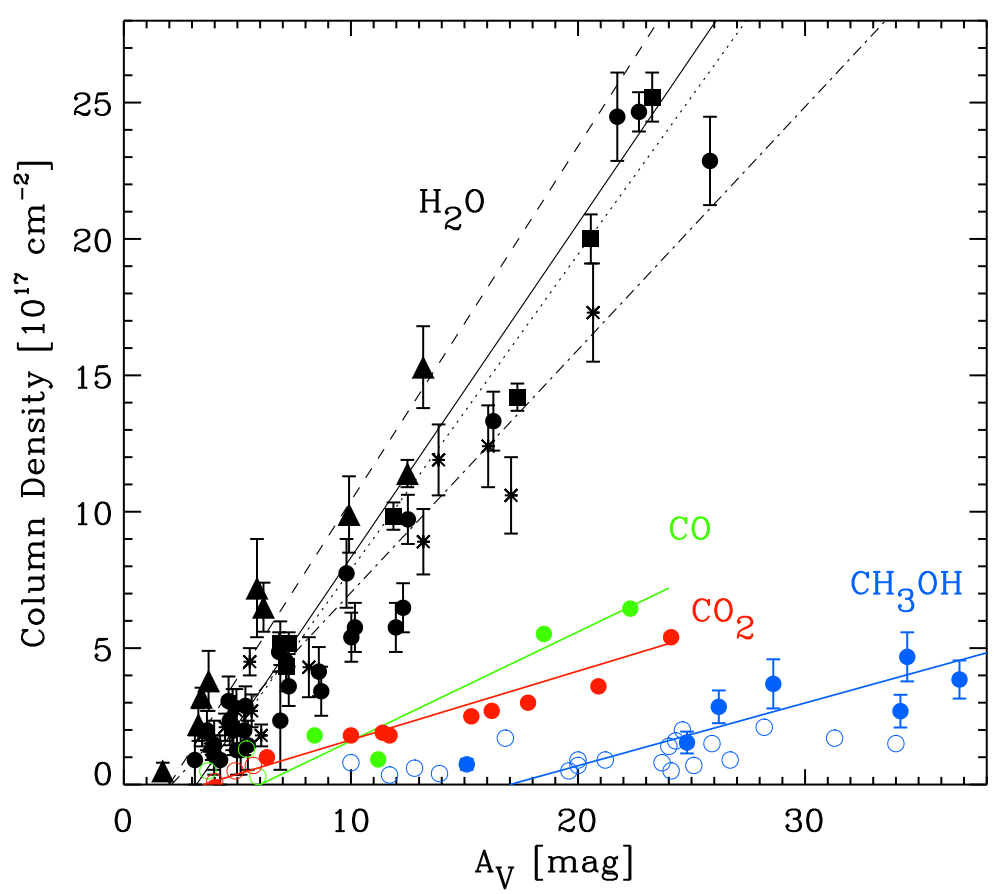

Figure 7: Relation between $\mathrm{H}_{2} \mathrm{O}$ (black), $\mathrm{CO}$ (green), $\mathrm{CO}_{2}$ (red), and $\mathrm{CH}_{3} \mathrm{OH}$ (blue) ice column densities and extinction $A_{\mathrm{V}}$ for quiescent lines of sight in nearby clouds and cores. Upper limits are indicated by open symbols. The lines represent linear fits to the detections. For $\mathrm{H}_{2} \mathrm{O}$, data points are shown for the Taurus (circles and solid line) and Lupus IV (asterisks and dash-dot line) clouds, as well as the L183 (triangles and dashed line) and IC 5146 (squares and dotted line) cores. For CO and $\mathrm{CO}_{2}$, the data points are for Taurus only, and for $\mathrm{CH}_{3} \mathrm{OH}$ they are for a variety of dense cores. The data were taken from Chiar et al. (1995), Whittet et al. (2007), Boogert et al. (2011), Chiar et al. (2011), Whittet et al. (2013) and Boogert et al. (2013).

published since the launch of Spitzer Space Telescope in 2003, but the earlier data have been re-analyzed with new methods or included as comparison objects with LYSOs in later work (Pontoppidan et al., 2008; Boogert et al., 2008). MYSOs in the GC region are discussed in $\$ 5.5$.

The evidence for thermal processing of the MYSO ices is ubiquitous in all the tracers discussed in 4 . This is mostly based on observations along a single line of sight toward the central object, but ice maps have confirmed it. Using extended scattered light as a background source, the $3.0 \mu \mathrm{m}$ band is deep toward a disklike structure around the MYSO AFGL 2136, but the ice abundance is suppressed relative to the surrounding cloud (Holbrook \& Temi, 1998), probably as a result of sublimation. Similar conclusions were drawn for ice maps toward the MYSO S140 IRS1 (Harker et al., 1997).

\subsection{Envelopes of Low and Intermediate Mass YSOs}

As the link between quiescent clouds and protoplanetary disks that give birth to potentially life-bearing planetary systems, ices in the envelopes of LYSOs are of paramount importance. The first $(3.0 \mu \mathrm{m})$ ice band toward an LYSO was detected 
by Cohen (1975) toward HL Tau and it was thought to originate in an edge-on disk (Cohen, 1983), but a location in the envelope is more likely (Furlan et al.,, 2008). This demonstrates the difficulty of locating ices along the line of sight (Boogert et al., 2002c). Here, surveys of ices most likely located in envelopes around LYSOs are reviewed, while disk studies are reviewed in $\$ 5.4$.

Comprehensive surveys of ices in the envelopes of embedded LYSOs were enabled by the Spitzer mission (5-20 $\mu \mathrm{m})$ and sensitive ground-based 3-5 $\mu \mathrm{m}$ instruments. The combination of the observations proved critical in the separation of overlapping bands (e.g., in the 5-8 $\mu \mathrm{m}$ region; 6.16 ), in obtaining stronger constraints on the ice mantle composition (e.g., unraveling the $15.2 \mu \mathrm{m} \mathrm{CO}_{2}$ band profile; 4.3), and in obtaining a complete inventory. Samples of $\sim 45$ objects in nearby clouds (Perseus, Serpens, Corona Australis, Taurus, Oph) and several cores were studied in the 5-8 $\mu \mathrm{m}$ and $\mathrm{H}_{2} \mathrm{O}$ features (Boogert et al., 2008) and the bands of $\mathrm{CO}$ and $\mathrm{CO}_{2}$ (Pontoppidan et al., 2003, 2008), $\mathrm{CH}_{4}$ (Öberg et al., 2008), $\mathrm{CH}_{3} \mathrm{OH}$ and $\mathrm{NH}_{3}$ (Bottinelli et al., 2010), and "XCN" (van Broekhuizen et al., 2005). A survey of all ice absorptions in the 5-20 $\mu \mathrm{m}$ range focused on LYSOs in Taurus was done as well (Zasowski et al., 2009).

These studies consider mostly Class 0 and I LYSOs. As for MYSOs, variations in abundances and processing history were observed. Thermal processing was found to play an important role, despite the lower stellar luminosities. Aided by episodic heating events, sublimation of the most volatile $\mathrm{CO}$ and $\mathrm{CO}_{2}$ components was observed for even the lowest luminosity YSOs $\left(<0.1 \mathrm{~L}_{\odot}\right.$; Kim et al. 2012). Large variations of the $\mathrm{OCN}^{-}$abundance $(4.64 \mu \mathrm{m})$, even for sources close together on the sky, suggest special conditions in envelopes (van Broekhuizen et al., 2005). They may be related to past CO freeze-out and possibly heating events (88)

LYSOs in clustered and high radiation environments of nearby massive stars may be more representative of the early solar system than isolated YSOs (Adams, 2010). An example is the IC 1396 globule irradiated by a nearby O-star. It shows $6.85 \mu \mathrm{m}$ features peaking at relatively long wavelengths (Reach et al., 2009), a high temperature effect that is rarely observed toward isolated LYSOs (\$4.4).

Finally, observations of the envelopes of intermediate mass YSOs (masses of 2$10 \mathrm{M}_{\odot}$ and luminosities of a few to $10^{4} \mathrm{~L}_{\odot}$ ) would enable further studies of the dependency of ice characteristics on stellar mass, but the sample sizes are small. In a Spitzer survey of 14 embedded intermediate mass YSOs distributed on the sky, Pitann et al. (2011) showed that the common ice features are present and that the $15.2 \mu \mathrm{m} \mathrm{CO} \mathrm{CO}_{2}$ band shows no signs of segregation. Thermal processing is, however, evident by reduced $\mathrm{H}_{2} \mathrm{O}$ and $\mathrm{CO}$ ice abundances in intermediate mass YSOs in the Vela cloud (Thi et al., 2006). One of these sources has high $\mathrm{CH}_{3} \mathrm{OH}$ and $\mathrm{OCN}^{-}$ abundances, but this is not necessarily a sign of thermal processing ( $\$ 6.1$ and 8.1 ).

\subsection{Disks Around Low and Intermediate Mass YSOs}

Direct observations of ices in circumstellar disks trace the volatile building blocks of planet and comet formation. These observations are challenging because the relatively flat disk geometry requires a specific orientation for the 2-20 $\mu \mathrm{m}$ vibrational modes to be seen in absorption. Great improvements in the sensitivity and spatial resolution of infrared observations, as well as in disk models, has led to much 
progress, however. A recurring conclusion is that ices in disks are strongly affected by thermally processing.

For flared disks, the observed ice features are most prominent if the disk inclination is comparable to the opening angle of about 70 degrees (Pontoppidan et al., 2005). Lower angles trace less dense outer disk regions and the observed ice features are also diluted as the size of background scattered light region exceeds that of the absorbing region. At higher angles, the high disk mid-plane densities increase the dust optical depths. As a result, ice observations are biased toward processed ices in the disk surface layers. This is indeed observed in the edge-on disk source CRBR 2422.8-3423 in Oph (Pontoppidan et al., 2005), as it is one of the few low mass sources with a shifted $6.85 \mu \mathrm{m}$ feature indicative of high temperature processing (\$4.4).

Rather than using the light from the central object, Terada \& Tokunaga (2012a) and Terada et al. (2012b) use the extended background of the Orion nebula to trace the ices. This work confirms the optimal disk inclination angle of 65-75 degrees needed to detect ice features. One source shows an unprecedented $\mathrm{H}_{2} \mathrm{O}$ ice band profile (Fig. 3i) with a strong contribution from large $(\sim 0.8 \mu \mathrm{m})$ grains rich in crystalline ices that may have surfaced from the lower disk regions where coagulation is more efficient. The same authors have resolved a (different) edge-on disk against the extended background and find spatial variations in band depth but not shape. The depth does not vary on a time scale of 4 years (Terada et al., 2012b), implying that the crystalline ices are extended over at least $3 \mathrm{AU}$ at distances beyond $30 \mathrm{AU}$. This distance estimate comes from spatially resolved spectroscopy by Schegerer \& Wolf (2010) toward the T Tau object YLW 16A.

High spatial resolution observations have indeed been very powerful in locating ices in disks. Coronographic adaptive optics ( $\mathrm{AO})$ images in narrow band filters of the Herbig Ae star HD 142527 reveal a spatially extended $3 \mu \mathrm{m}$ absorption attributed to scattering by icy $\sim 1 \mu \mathrm{m}$ sized grains in the disk (Honda et al., 2009). Also, AO spectra separated the HK Tau and HV Tau binary and tertiary systems, revealing deep (amorphous) ice bands in the HK Tau B and HV Tau C edge-on disks. The depth toward HV Tau C varies on a time scale of 2.3 years, which could be caused by a 1.4 AU sized structure at a distance of $100 \mathrm{AU}$ from the star (Terada et al., 2007).

A particularly successful technique to trace ices in circumstellar disks, that is less dependent on the disk orientation, is spectroscopy of the far-infrared ice lattice modes (\$4.5). In passively heated, flared disks, the lattice modes of $\mathrm{H}_{2} \mathrm{O}$ ice at 44 and $62 \mu \mathrm{m}$ are excited in super-heated optically thin disk surfaces at radii beyond $100 \mathrm{AU}$ (Chiang et al., 2001). In this layer, the ice condensation radius (not to be confused with the "snow line" in the disk mid-plane) scales with the 3rd power of the stellar temperature, which explains the detection of the lattice modes in ISO spectra of the coolest members of their sample of Herbig AeBe and T Tauri stars.

The ISO spectrometers detected more of the lattice modes, mostly from crystalline $\mathrm{H}_{2} \mathrm{O}$, in bright disks around intermediate mass stars: the isolated Herbig Ae stars HD 100546 (Malfait et al., 1998) and HD 142527 (Malfait et al., 1999), and the Herbig Ae star HD 163296, but not AB Aur (van den Ancker et al., 2000). Observations with the Herschel/PACS spectrometer are limited by calibration uncertainties and incomplete coverage of the strongest $(44 \mu \mathrm{m})$ lattice mode. Detections claimed for a few $\mathrm{T}$ Tauri stars indicate a high degree of crystallinity created by past heating events, such as planetesimal collisions (McClure et al., 2015). 
Finally, sensitive, but low spatial resolution Akari observations of a sample of edgeon disk sources confirm the optimal inclination that is needed to detect ice features, but the spectral resolution is too low to study processing effects (Aikawa et al., 2012). Their 2-5 $\mu \mathrm{m}$ spectra reveal solid $\mathrm{H}_{2} \mathrm{O}, \mathrm{CO}, \mathrm{OCN}^{-}$, OCS, and possibly HDO.

\subsection{Galactic Center Region}

The conditions in the GC region differ significantly from those elsewhere in the Galaxy (elemental abundances, radiation fields, dynamic time scales) and thus offer a unique perspective on ice formation and evolution processes. Studies of ice features in the GC region have primarily targeted the cluster of infrared sources (GC IRS) within a parsec from $\mathrm{SgrA}^{*}$, and MYSOs (candidates) throughout an extended $(\sim 50 \times 200$ pc) region in the Central Molecular Zone (CMZ).

Most of the infrared sources in the central parsec of the Galaxy show ice absorption features. The depths of the $3.0 \mu \mathrm{m} \mathrm{H}_{2} \mathrm{O}$ and $4.67 \mu \mathrm{m} \mathrm{CO}$ bands (McFadzean et al., 1989; Chiar et al., 2002; Moultaka et al., 2005) and the $4.62 \mu \mathrm{m}$ XCN feature (Moneti et al., 2001; Chiar et al., 2002; Moultaka et al., 2009) vary with factors of $\sim 4$ between these sources. As much as $60-80 \%$ of the $\mathrm{H}_{2} \mathrm{O}$ ices may be local to the inner pc of the Galaxy, possibly in dense clumps in the circum-nuclear ring or mini-spiral (Chiar et al., 2002; Moultaka et al., 2005). The foreground contamination is less certain for CO ices (Moultaka et al., 2009), but in any case the total column relative to $\mathrm{H}_{2} \mathrm{O}$ is small $(<10 \%)$. While not much $\mathrm{CO}$ has frozen out, there is little evidence for thermal processing. The CO profile has a strong apolar component (Moneti et al., 2001; Moultaka et al., 2005), and the $15.2 \mu \mathrm{m} \mathrm{CO} \mathrm{CO}_{2}$ bending mode, observed in the ISO beam of $\sim 20$ " encompassing a number of IRS sources, shows a lack of thermal processing as well (Gerakines et al., 1999). Other detected ice species include $\mathrm{NH}_{3}$ and $\mathrm{CH}_{4}$, while solid $\mathrm{CH}_{3} \mathrm{OH}$ is notably under-abundant $(<5 \%$; Chiar et al. 2000 , 2002). The detection of the stretch mode of $\mathrm{NH}_{3}$ at $2.96 \mu \mathrm{m}$ in ISO and high spatial resolution ground-based observations, supported by the presence of $\mathrm{NH}_{3} \cdot \mathrm{H}_{2} \mathrm{O}$ hydrates at $3.2-3.7 \mu \mathrm{m}$ and by the $6.2 \mu \mathrm{m}$ bend mode, is unusual and implies a large $\mathrm{NH}_{3}$ abundance of up to $30 \%$ relative to solid $\mathrm{H}_{2} \mathrm{O}$. An N-rich environment seems also reflected in a high $\mathrm{OCN}^{-}$abundance of $3 \%$ toward GC IRS 19 (Chiar et al. 2002, recalculated using the band strength in van Broekhuizen et al. 2005).

On a larger scale, ices were detected in numerous sources throughout the CMZ. In a sample of 107 targets selected using 2MASS and Spitzer 1-8 $\mu \mathrm{m}$ broad band photometry, 35 were identified as MYSOs (or candidates; 8-23 $M_{\odot}$ ) following Spitzer spectroscopy (An et al., 2011). They are located throughout the CMZ, although there is clustering near the Sgr B molecular complex. All objects show ice features in the 5-7 $\mu \mathrm{m}$ region, as well as the $15.2 \mu \mathrm{m} \mathrm{CO}_{2}$ bending mode. The latter was used as a criterion in the classification of the sources as MYSOs: the presence of a long wavelength wing tracing the $\mathrm{CH}_{3} \mathrm{OH}: \mathrm{CO}_{2}$ complex was required (Fig. 5). It is based on the fact that until then this wing had only been detected towards Galactic Disk MYSOs with high $\mathrm{CH}_{3} \mathrm{OH}$ abundances (4.3). Further work is needed to confirm the nature of these sources and the location of the ices in the MYSO envelopes or in the dense cloud medium in the CMZ. Also, spectra at wavelengths shorter than 5 $\mu \mathrm{m}$ have not yet been obtained, thus direct measurements of the $\mathrm{H}_{2} \mathrm{O}, \mathrm{CH}_{3} \mathrm{OH}$, and $\mathrm{CO}$ abundances toward the CMZ MYSOs are lacking. 


\subsection{Shells Surrounding Evolved Stars}

Certain classes of evolved stars show $\mathrm{H}_{2} \mathrm{O}$ ice bands and offer a view on ice properties from a completely different perspective compared to that of the ISM and YSOs. In general, these are carbon-poor AGB stars with very high mass loss rates, showing $\mathrm{OH}$ maser emission (OH/IR stars), or the slightly more evolved post-AGB stars that still have dense, but expanded and cooled envelopes. In short, the expanding dense shells of gas expelled by evolved stars cool rapidly. At some point, $\mathrm{H}_{2} \mathrm{O}$ is formed in the warm gas, and as the shell cools it condenses onto the refractory grains formed in an earlier, hotter phase. The $3.0 \mu \mathrm{m}$ band is narrower in $\mathrm{OH} / \mathrm{IR}$ stars compared to YSOs (Fig. 3, Gillett \& Soifer 1976) and is thought to reflect the purity of the $\mathrm{H}_{2} \mathrm{O}$ ice, its crystallinity, and the small grain size (Smith et al., 1988). The 6.0 bending mode (Soifer et al., 1981), the $11 \mu \mathrm{m}$ libration mode (Sylvester et al., 1999) and the lattice modes at 44 and $62 \mu \mathrm{m}$ were detected as well. The lattice mode was first detected by anomalously strong IRAS $60 \mu \mathrm{m}$ broad band emission, half of which can be attributed to $\mathrm{H}_{2} \mathrm{O}$ ice (Forveille et al., 1987; Omont et al., 1990), in the postAGB star Frosty Leo. The lattice modes strongly confirm the crystallinity of the ice (Omont et al., 1990; Sylvester et al., 1999; Demvk et al., 2000). No other ice species were detected toward evolved stars (Gillett \& Soifer, 1976; Sylvester et al., 1999), in part due to the oxygen rich environment in which they were formed.

\subsection{Massive YSOs in the Magellanic Clouds}

Observations of ices in the Magellanic Clouds offer unique tests of ice and molecule formation and evolution models, due to the lower metallicities and correspondingly lower dust content and higher UV fields. Overall, differences in the relative ice abundances and higher levels of processing have been found relative to Galactic ices.

The $\mathrm{CO}_{2} / \mathrm{H}_{2} \mathrm{O}$ column density ratios are a factor of two larger in the envelopes of MYSOs $\left(10^{4}-10^{5} L_{\odot}\right)$ in the Large Magellanic Cloud (LMC; $33 \pm 1 \%$; Seale et al. 2011 using data from Shimonishi et al. 2008; Oliveira et al. 2009; Shimonishi et al. 2010) compared to the Galaxy (17 $\pm 3 \%$; Gerakines et al. 1999). The shape of the $15.2 \mu \mathrm{m} \mathrm{CO} \mathrm{CO}_{2}$ band is very well studied ( 6.3 , Seale et al. 2011; Oliveira et al. 2009). In the majority of sightlines, $\mathrm{CO}_{2}$ ices have experienced thermal processing. $\mathrm{CO}$ ice is detected in ground-based spectra (Oliveira et al., 2011) and in low resolution Akari spectra (Shimonishi et al., 2010), indicating a low abundance $(\leq 15 \%$ relative to $\mathrm{H}_{2} \mathrm{O}$ ). The 5-7 $\mu \mathrm{m}$ ice features, including a clear $6.85 \mu \mathrm{m}$ feature in at least one object, were detected (Oliveira et al., 2009) although their analysis is severely hampered by overlapping PAH emission (Seale et al., 2009). The XCN feature at $4.62 \mu \mathrm{m}$ may have been detected in a few Akari sources but suffers from low spectral resolution (Shimonishi et al., 2010). This is the case for the $3.53 \mu \mathrm{m}$ band of solid $\mathrm{CH}_{3} \mathrm{OH}$ as well.

$\mathrm{H}_{2} \mathrm{O}$ and $\mathrm{CO}_{2}$ ices were also detected toward 14 MYSOs in the Small Magellanic Cloud (SMC) in a combined Spitzer and ground-based 3-4 $\mu \mathrm{m}$ survey (Oliveira et al., 2013 ). The relative column densities are compared to other environments in 6.3 , Heating effects play a role here as well, judging the presence of the $62 \mu \mathrm{m}$ lattice mode of crystalline $\mathrm{H}_{2} \mathrm{O}$ ice in Spitzer spectra (van Loon et al., 2010). The 6.0 and $6.8 \mu \mathrm{m}$ features were detected, but CO ice is absent at levels below those in the LMC 
(although only four lines of sight were observed; Oliveira et al. 2011).

\subsection{Other Galaxies}

Ice features are commonly seen in the infrared spectra of external galaxies with centers dominated by starbursts or dust-enshrouded Active Galactic Nuclei (AGN). They are often used to determine the nature of the emission sources. Imanishi \& Maloney (2003) show that peak optical depths of the $3.0 \mu \mathrm{m}$ band larger than $\sim 0.3$ must be caused by compact AGN emission surrounded by dust clouds containing the ices. Weaker ice features are due to starburst regions in which many MYSOs are mixed with clouds containing ice coated dust grains within the same beam. Local ULIRGS and most LINER-type galaxies show deep ice bands, and their centers are thus dustenshrouded AGN. In contrast, Seyfert-type galaxies do not show ice bands at all, lacking such dusty centers (Imanishi et al., 2006).

Besides $\mathrm{H}_{2} \mathrm{O}$ ice $(3.0$ and $6.0 \mu \mathrm{m})$, solid $\mathrm{CO}_{2}$, "XCN", $\mathrm{CO}$ and $\mathrm{CH}_{4}$ were detected in these galaxies (Spoon et al., 2000, 2004). The spectra often show a combination of PAH emission, and absorptions from diffuse dust and ices, much like the center of the Galaxy (Sturm et al., 2000), complicating the analysis of the ice features.

The ice distribution was mapped in several nearby galaxies, and enhanced relative abundances are often attributed to production in energetic radiation fields. Toward a ring encircling the starburst nucleus of NGC 4945, the $4.62 \mu \mathrm{m} \mathrm{XCN} \mathrm{feature}$ is stronger than that of CO, similar to that in the Galactic MYSO W33A. The depths do not vary much within the ring. Spoon et al. (2003) attribute it to UVprocessed ices (X-ray fields are likely too weak). The Akari satellite mapped the ice distributions in other starburst-dominated galaxies. In M82, variations of the $\mathrm{CO}_{2} / \mathrm{H}_{2} \mathrm{O}$ abundance ratio correlate with the UV field traced by $\mathrm{Br} \alpha$ emission, possibly indicating the energetic production of $\mathrm{CO}_{2}$ (Yamagishi et al., 2013). In view of the difficulty of identifying energetically-produced ices in Galactic environments ( 88.3$)$, these claims for nearby galaxies must be regarded as tentative.

The current redshift record for ice feature detections is 2.28 (Sajina et al., 2009). At such high redshift, both the 3 and $6 \mu \mathrm{m}$ ice bands are within the Spitzer spectrometer wavelength range. Overall, no differences in the ice composition or abundance were found as a function of redshift, but the data are limited.

\subsection{Solar System Objects}

There are many ice-rich environments in the Solar System, including the satellites of the gas- and ice-giant planets, trans-Neptunian Objects (TNOs), and comets. For this review of interstellar and circumstellar ices, cometary ices are of particular interest because of their possible link with ices in the ISM. Ice abundances are the main observables to test this link. While present day comets originate from distinct heliocentric distances, formation regions are less clear as a result of radial mixing. For example, organic-enriched comets are present in both Kuiper Belt and Oort Cloud comets. A certain population of comets may even be captured from sibling star systems formed from the same cluster as the Sun. Cometary ice abundances will not be reviewed here, but rather the review by Mumma \& Charnley (2011) will be used as a reference. Remarkable differences and similarities with respect to interstellar 
ices are observed $(\$ 6.5)$.

\section{COLUMN DENSITIES AND ABUNDANCES}

Ice column densities are generally determined by dividing the integrated optical depth by the integrated band strength $A$ measured in laboratory experiments (\$3):

$$
N=\int \tau_{\nu} d \nu / A\left[\mathrm{~cm}^{-2}\right]
$$

For saturated bands, and in particular for unresolved ones such as those observed with the low resolution mode of the Akari satellite $(R \sim 20)$, a curve of growth analysis must be performed to derive the column density (Shimonishi et al., 2008). For LYSOs with disks, the size of the background region of scattered light, and thus the observed ice column in the foreground, may be a strong function of wavelength (Pontoppidan et al., 2005). Different modes of the same species would then yield different column densities, and in fact their ratio would trace the disk inclination. So far this has not been observationally demonstrated, however. Similar scenarios were suggested to explain discrepancies between the 3 and $6 \mu \mathrm{m}$ absorption band depths towards MYSOs due to $\mathrm{H}_{2} \mathrm{O}$ (Keane et al., 2001b), but this also still remains to be proven. For example, toward the MYSO S140 IRS1, the column densities derived for all $\mathrm{CO}_{2}$ bands over the $2-15 \mu \mathrm{m}$ wavelength range are in good agreement (Keane et al., 2001a). Finally, for external galaxies such as ULIRGs and starburst galaxies, a single spatial resolution element may contain multiple continuum sources (e.g., YSOs, an AGN), and depending on the geometry of those and the dense clouds containing ices, the ice band depths may be quite different (4.1, Imanishi \& Maloney 2003 ).

Equation 11 only applies to pure absorption. For features caused by scattering (\$2), the ice column traced depends strongly on the grain size, and radiative transfer involving grain size distributions and appropriate optical constants must be calcu-

MRN: number density distribution of grain radii derived from diffuse cloud observations, $\mathrm{n}(\mathrm{a}) \propto$ $a^{-3.5}$ (Mathis et al., 1977).

\section{Upper and lower} quartile abundances: median of the abundances respectively larger and less than the median of all abundances. lated (Pendleton et al., 1990; Dartois \& d'Hendecourt, 2001). Similarly, the lattice modes $(>25 \mu \mathrm{m})$ may appear in emission and radiative transfer models are required to evaluate column densities such as demonstrated for disks surrounding YSOs (Chiang et al., 2001) and envelopes surrounding MYSOs (Dartois et al., 1998).

$\mathrm{H}_{2} \mathrm{O}$ ice was detected at column densities ranging from $\sim 10^{17}$ to $10^{19} \mathrm{~cm}^{-2}$. The lower limit corresponds to a peak optical depth of the $3.0 \mu \mathrm{m}$ band of 0.06 , which is rather high because of uncertainties in the continuum determination. It corresponds to dust extinctions close to the ice formation threshold (\$5.1) and thus to a few monolayers of ice on each grain in the MRN grain size distribution with a lower limit of $20 \AA$ (Hollenbach et al., 2009). $\mathrm{H}_{2} \mathrm{O}$ might be present on grains below the ice formation threshold, but, besides the low column, the observational signature is weakened by the much lower band strength of isolated water compared to that of bulk ice (\$4.1).

Ice abundances relative to $\mathrm{H}_{2} \mathrm{O}, X_{\mathrm{H}_{2} \mathrm{O}}$, were determined following the statistical method in Öberg et al. (2011), but using somewhat different samples and including minor ice species. The values for the Galactic sources are listed in Table 2, For samples larger than a few, the median and lower and upper quartiles of the abundance distributions are given, as well as the median abundances taking into account upper limits. Also listed are the full ranges of the observed abundances. 
Cometary abundances derived from measured gas phase production rates were taken from Mumma \& Charnley (2011), supplemented by measurements of $\mathrm{CO}_{2}$ abundances with the Akari satellite (within 2.7 AU, the $\mathrm{H}_{2} \mathrm{O}$ sublimation radius; Ootsubo et al. 2012). Considerable variations are observed within and across environments $(\underline{6.3})$. The insecurely identified species are discussed in $\$ 6.1$, and upper limits for other astrochemically relevant species in 6.2 . 
Table 2: Ice Abundances

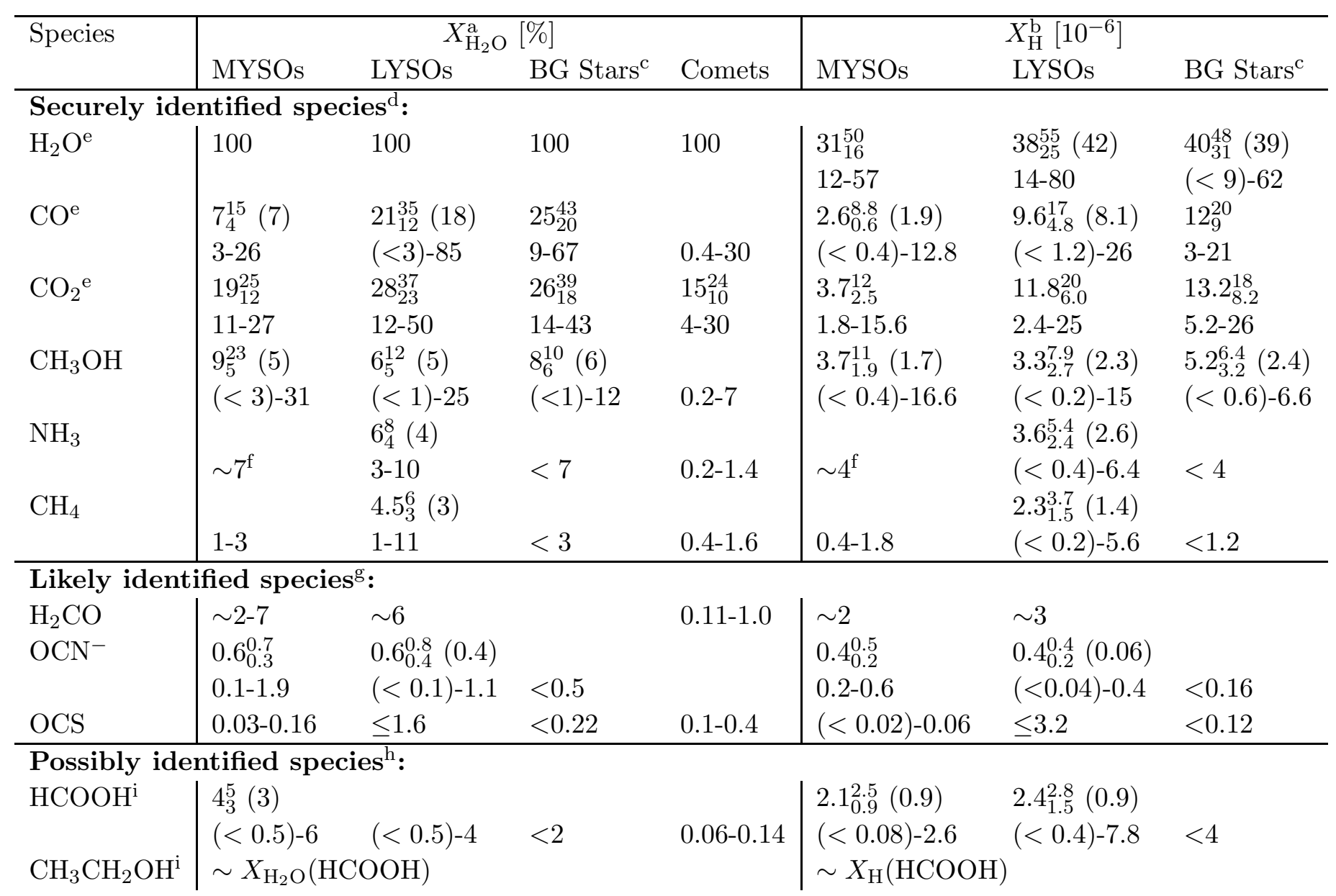




\begin{tabular}{|c|c|c|c|c|c|c|c|}
\hline \multirow[t]{2}{*}{ Species } & \multicolumn{4}{|c|}{$X_{\mathrm{H}_{2} \mathrm{O}}^{\mathrm{a}}[\%]$} & \multicolumn{3}{|c|}{$X_{\mathrm{H}}^{\mathrm{b}}\left[10^{-6}\right]$} \\
\hline & MYSOs & LYSOs & BG Stars ${ }^{\mathrm{c}}$ & Comets & MYSO & LYSOs & BG Stars $^{c}$ \\
\hline $\mathrm{HCOO}^{-\mathrm{j}}$ & \multicolumn{4}{|l|}{$0.5_{0.5}^{0.7}(0.5)$} & \multicolumn{3}{|c|}{$0.18_{0.12}^{0.28}(0.14)$} \\
\hline & $0.3-1.0$ & $\sim 0.4$ & $<0.1$ & & $(<0.10$ & $\sim 0.2$ & $<0.8$ \\
\hline $\mathrm{CH}_{3} \mathrm{CHO}^{\mathrm{j}}$ & \multicolumn{4}{|c|}{$X_{\mathrm{H}_{2} \mathrm{O}}\left(\mathrm{HCOO}^{-}\right) \times 11$} & \multicolumn{3}{|c|}{$X_{\mathrm{H}}\left(\mathrm{HCOO}^{-}\right) \times 11$} \\
\hline \multirow{2}{*}{$\mathrm{NH}_{4}^{+}$} & $11_{9}^{13}$ & $11_{7}^{15}$ & $8_{6}^{11}$ & & $4.1_{1.8}^{5.4}$ & $4.6_{3.2}^{5.8}$ & $3.8_{2.9}^{4.9}$ \\
\hline & $9-34$ & $4-25$ & $4-13$ & & $1.4-6.0$ & $0.8-12$ & $1.9-9.6$ \\
\hline $\mathrm{SO}_{2}$ & $(<0.9)-1.4$ & $\sim 0.2$ & & 0.2 & $\leq 0.4$ & $\sim 0.08$ & \\
\hline $\mathrm{PAH}^{\mathrm{k}}$ & $\sim 8$ & & & & $\sim 1$ & & \\
\hline
\end{tabular}

${ }^{a}$ Abundances relative to the $\mathrm{H}_{2} \mathrm{O}$ ice column density. For each molecule, the first row gives the median and lower and upper quartile values of the detections, and in brackets the median including upper limits. The second row gives the full range of abundances. If only one row is given, it shows the full range. See Table 1 and $₫ 6$ for references.

b As in the $X_{\mathrm{H}_{2} \mathrm{O}}$ column, but for abundances relative to $N_{\mathrm{H}}$.

c Quiescent clouds and cores.

${ }^{\mathrm{d}}$ Identified based on multiple modes or isotopologues in high quality spectra.

e The isotopologues ${ }^{13} \mathrm{CO}$ and ${ }^{13} \mathrm{CO}_{2}$ were also securely identified, and $\mathrm{HDO}$ was possibly identified (乌6.4).

${ }^{\mathrm{f}}$ Values derived from the simultaneous analysis of multiple features (Dartois et al., 2002).

g Identification based on a single absorption feature and the profile matches laboratory spectra.

${ }^{\mathrm{h}}$ Identification based on a single absorption feature and no convincing match to laboratory spectra is available.

${ }^{\mathrm{i}} \mathrm{HCOOH}$ and/or $\mathrm{CH}_{3} \mathrm{CH}_{2} \mathrm{OH}$ may be carriers of the $7.24 \mu \mathrm{m}$ ice band (Schutte et al., 1999; Öberg et al., 2011).

j $\mathrm{HCOO}^{-}$and/or $\mathrm{CH}_{3} \mathrm{CHO}$ may be carriers of the $7.41 \mu \mathrm{m}$ ice band (Schutte et al., 1999).

${ }^{\mathrm{k}}$ Using column density of C-C bonds derived in Hardegree-Ullman et al. (2014) and assuming each PAH species contains 50 carbon atoms. 
Ice abundances relative to elemental hydrogen, $X_{\mathrm{H}}$, are generally calculated by determining $N_{\mathrm{H}}=N(\mathrm{HI})+2 N\left(\mathrm{H}_{2}\right)$ from scaling relations with the silicate feature $\left(\tau_{9.7}\right)$ or the extinction in the near-infrared $\left(A_{\mathrm{K}}\right)$ or visual $\left(A_{\mathrm{V}}\right)$. These abundances are also given in Table 2, assuming $\tau_{9.7}=0.26 A_{\mathrm{K}}$ (Boogert et al., 2013) and $N_{\mathrm{H}}=1.54 \times 10^{22} A_{\mathrm{K}}$ (Vuong et al., 2003). Another way to express ice abundances, of particular interest to planetary disk models, is the ice-to-rock mass ratio. Defining "rock" as the silicate dust component, the highest observed ice abundances correspond to ice-to-rock ratios of $\sim 1.5$ (Pontoppidan et al., 2014).

Finally, for extragalactic sources only $\mathrm{CO}, \mathrm{CO}_{2}$, and $\mathrm{H}_{2} \mathrm{O}$ ices were observed in more than a few sightlines. Following the notation of Table 2, $X_{\mathrm{H}_{2} \mathrm{O}}(\mathrm{CO})$ is $9_{8}^{18}((<$ 4)-19) for the LMC (Shimonishi et al., 2010; Oliveira et al., 2011), and $<3$ for the SMC (Oliveira et al., 2011). $X_{\mathrm{H}_{2} \mathrm{O}}\left(\mathrm{CO}_{2}\right)$ is $17_{9}^{25}(6-41)$ for the SMC (Oliveira et al., 2013 ) and $36_{29}^{39}(10-46)$ for the LMC (Shimonishi et al., 2010; Seale et al., 2011; Oliveira et al., 2009; Shimonishi et al., 2008). Abundances relative to hydrogen were not published. The Magellanic Cloud abundances are compared with Galactic environments in 6.3 .

\subsection{Identification Issues}

The origin of a number of ice absorption features is uncertain (Table 1). As opposed to gas phase features (Herbst \& van Dishoeck, 2009), the identification of ice features is complicated by the dependence of the peak position on the ice environment (\$3 and 4). Therefore, Table 2 is divided into categories of "securely", "likely", and "possibly" identified carriers. For securely identified species, more than one vibrational mode was detected and the depth and profile were successfully fitted with models and laboratory spectra. Although CO ice has only one mode $(4.67 \mu \mathrm{m})$, the ${ }^{13} \mathrm{CO}$ isotopologue at $4.78 \mu \mathrm{m}$ was detected as well. Species in the "likely identified" category have only one securely detected absorption band, but an excellent fit to the profile and position was demonstrated and the abundance is reasonably well understood. Species in the "possibly identified" category lack a convincing profile fit or would have an unreasonably high abundances (e.g., compared to gas phase measurements). $\mathrm{SO}_{2}$ is in this category as well, because, although the $7.63 \mu \mathrm{m}$ feature was well fitted by $\mathrm{SO}_{2}$ in $\mathrm{CH}_{3} \mathrm{OH}$ ices, it is so far detected in only one line of sight. In the remainder of this section some identification issues are highlighted.

PAHs: Polycyclic Aromatic Hydrocarbon molecules, responsible for the ubiquitous infrared emission features, and, in dense environments, likely also frozen out in ice mantles.
The 3.25 $\mu \mathrm{m}$ (PAH?) feature. The $3.25 \mu \mathrm{m}$ absorption feature depth, measured toward a handful of YSOs, was found to correlate better with the 9.7 than the $3.0 \mu \mathrm{m}$ band depth, consistent with a more refractory carrier (Brooke et al., 1999). The $\mathrm{C}-\mathrm{H}$ stretch mode of $\mathrm{PAH}$ species, detected by its $3.29 \mu \mathrm{m}$ emission throughout the Galaxy, is thus a good candidate (Sellgren et al., 1994). Indeed, the strengths of other absorption features in YSO spectra, in particular in the 5-7 $\mu \mathrm{m}$ region, are consistent with PAHs embedded in the ices (Hardegree-Ullman et al., 2014). No convincing overall laboratory fit is available, however, due to the heterogeneity of PAH species, including size distributions and ionization within the ice (Bouwman et al., 2011). An alternative proposed carrier is $\mathrm{NH}_{4}^{+}$, which is also less volatile than $\mathrm{H}_{2} \mathrm{O}$ ices (Schutte \& Khanna, 2003), and whose spectrum also matches the $6.85 \mu \mathrm{m}$ feature (see below). 
The $3.47 \mu \mathrm{m}\left(\mathrm{NH}_{3}\right)$ feature. This feature, not to be confused with the $3.40 \mu \mathrm{m}$ feature due to saturated hydrocarbons only found in the diffuse ISM, was originally tentatively attributed to nano-diamonds (Allamandola et al., 1992) in dense clouds. A very good correlation of the 3.47 and $3.0 \mu \mathrm{m}$ band depths indicates a volatile nature of the carrier, however (Chiar et al.,, 1996; Brooke et al., 1999). Independent evidence came from spectropolarimetry, showing that this feature is polarized at a similar strength as the $3.0 \mu \mathrm{m}$ band (Hough et al., 1996). It is consistent with an origin in ammonia hydrates (\$4.1; Dartois \& d'Hendecourt 2001; Dartois et al. 2002). This is considered the preferred carrier, because it also explains much of the overall long-wavelength wing of the $3.0 \mu \mathrm{m}$ band and other $\mathrm{NH}_{3}$ features throughout the infrared spectra of YSOs.

The 4.62 $\mu \mathrm{m}$ ("XCN") feature. The $4.62 \mu \mathrm{m}$ feature was attributed to a carrier with a $\mathrm{C} \equiv \mathrm{N}$ (triple bond) group since its first detection (Lacy et al., 1984). Laboratory experiments in which CO-bearing ices containing $\mathrm{NH}_{3}$ were photolyzed reproduced this feature (d'Hendecourt et al., 1986) and pointed to the $\mathrm{C} \equiv \mathrm{N}$ bond of a small molecule. Hence, the observed absorption became known as "the XCN feature", but many laboratory studies have pointed to the true carrier as the ionic species $\mathrm{OCN}^{-}$(Demyk et al. 1998 and references therein). A good correlation between the gas phase HNCO, presumably photodesorbed at cloud edges, and solid state $\mathrm{OCN}^{-}$abundances supports this (Öberg et al., 2009a). Later it was shown that the interstellar absorption is a composite of features peaking at 4.60 and 4.64 $\mu \mathrm{m}$ (Pontoppidan et al., 2003). Only the peak position and profile of the $4.64 \mu \mathrm{m}$ component are in agreement with the $\mathrm{OCN}^{-}$species, most likely in polar ices that have possibly been heated (van Broekhuizen et al., 2005). It thus appears that its strength relative to apolar ices is an indicator of thermal processing, but only for environments that have experienced the "catastrophic" CO freeze out stage in which HNCO and subsequently $\mathrm{OCN}^{-}$were formed (8.1, Öberg et al. 2011). The nature of the $4.60 \mu \mathrm{m}$ component is less clear. Pontoppidan et al. (2003) suggest CO in a different binding site, and Öberg et al. (2011) suggest $\mathrm{OCN}^{-}$in apolar ices although no good fit was obtained for any mixture considered by van Broekhuizen et al. (2005).

\section{The 6.0 and $6.85 \mu \mathrm{m}$ (salts, organic residue, $\mathrm{PAH}, \mathrm{NH}_{4}^{+}$?) features.}

Securely identified species do not fully explain the observed prominent 6.0 and 6.85 $\mu \mathrm{m}$ absorption features in most sightlines (Fig. 6). Dilution of $\mathrm{H}_{2} \mathrm{O}$ in other species, such as the abundant $\mathrm{CO}_{2}$, enhances the strength of the $\mathrm{H}_{2} \mathrm{O}$ bending mode relative to the $3.0 \mu \mathrm{m}$ band (Knez et al., 2005), but this is generally insufficient to explain all $6.0 \mu \mathrm{m}$ absorption. HCOOH could be an important contributor but its identification in the ices is uncertain (see below). The carrier of the $6.85 \mu \mathrm{m}$ feature is not related to the bending modes of aliphatic hydrocarbons seen at similar wavelengths in the diffuse ISM due to the absence of the corresponding stretching modes at $3.40 \mu \mathrm{m}$. The $\mathrm{CO}_{3}^{-}$ion in minerals (carbonates, e.g., $\mathrm{MgCO}_{3}$ or $\mathrm{CaCO}_{3}$ ) is also excluded due to the absence of features at longer wavelengths in the spectra of YSOs (Schutte et al., 1996) and the excellent correlation between the depths of the $6.85 \mu \mathrm{m}$ absorption and known ice features (Tielens \& Allamandola, 1987a). The latter argument also excludes other refractory carriers. The $\mathrm{NH}_{4}^{+}$ion in salts (Knacke et al., 1982), is considered the most likely carrier, but it lacks a convincing profile fit. While the observed strengths correlates very well with $\mathrm{H}_{2} \mathrm{O}$ ice (Pontoppidan, 2006; 
Boogert et al., 2008), the laboratory profile is too broad in $\mathrm{H}_{2} \mathrm{O}$ ices (Gálvez et al., 2010). After $\mathrm{H}_{2} \mathrm{O}$ ice sublimation, a better fit is obtained, and at even higher temperature the peak position shifts to longer wavelengths, consistent with observations of YSOs (enhanced C4/C3 component ratios; 4.4). Finally, a very broad absorption observed toward a number of YSOs (C5 component; 4.4) has been ascribed to salts (Schutte \& Khanna, 2003) and organic residue (Gibb \& Whittet, 2002), both of which are energetic and/or thermal processing products, but these identifications need independent spectroscopic confirmation.

The $7.24 \mu \mathrm{m}$ (HCOOH?) feature. This feature was attributed to the C$\mathrm{H}$ deformation mode in $\mathrm{HCOOH}$, and specific mixtures containing both $\mathrm{H}_{2} \mathrm{O}$ and $\mathrm{CH}_{3} \mathrm{OH}$ are needed to match the peak position and profile (Schutte et al., 1999). The depth is consistent with the much stronger $\mathrm{C}=\mathrm{O}$ stretch mode at $5.85 \mu \mathrm{m}$ (Schutte et al., 1996), but its identification was put into question because of issues with the baseline in the laboratory spectra (Boogert et al., 2008; Öberg et al., 2011) and an abundance that is a factor of $10^{4}$ larger compared to the gas phase in hot cores is also not understood (Bisschop et al., 2007). Öberg et al. (2011) propose pure ethanol instead. Other species tend to have slightly blue-shifted peak positions $\left(\mathrm{HCONH}_{2}, \mathrm{C}_{5} \mathrm{H}_{12}\right.$; Schutte et al. 1999; $\mathrm{NH}_{2} \mathrm{CH}_{2} \mathrm{OH}$; Bossa et al. 2009) and are listed in Table 3 ,

\subsection{Upper Limits}

Table 3 shows abundance upper limits of astrophysically relevant species. These were determined by comparing laboratory spectra with the spectra of YSOs and background stars, taking into account position and profile dependencies on the ice composition. For symmetric species (in particular $\mathrm{O}_{2}, \mathrm{~N}_{2}$, and $\mathrm{H}_{2}$ ), interactions in the ice matrix induce a dipole moment, and the band strength is a very strong function of molecular environment. These make the derived abundance upper limits uncertain. Indirect measurements of the $\mathrm{O}_{2}$ and $\mathrm{N}_{2}$ abundances in mixtures with CO by the effect on the ${ }^{12} \mathrm{CO}$ (Elsila et al., 1997) and ${ }^{13} \mathrm{CO}$ (Boogert et al., 2002a) band profiles lower the upper limits for $\mathrm{N}_{2}$ by a factor of $\sim 5$, but do not further constrain those of $\mathrm{O}_{2}$. The case of $\mathrm{H}_{2}$ refers to energetically processed ices that are able to retain the produced $\mathrm{H}_{2}$ (Sandford et al., 1993 ). In pure form $\mathrm{H}_{2}$ sublimates at $3 \mathrm{~K}$, well below the temperature of dense clouds. An upper limit rather than the detection claimed in Sandford et al. (1993) is quoted because of confusion with photospheric absorption lines at $2.415 \mu \mathrm{m}$. 
Table 3: Abundance upper limits of astrochemically relevant ices and corresponding detections in comets

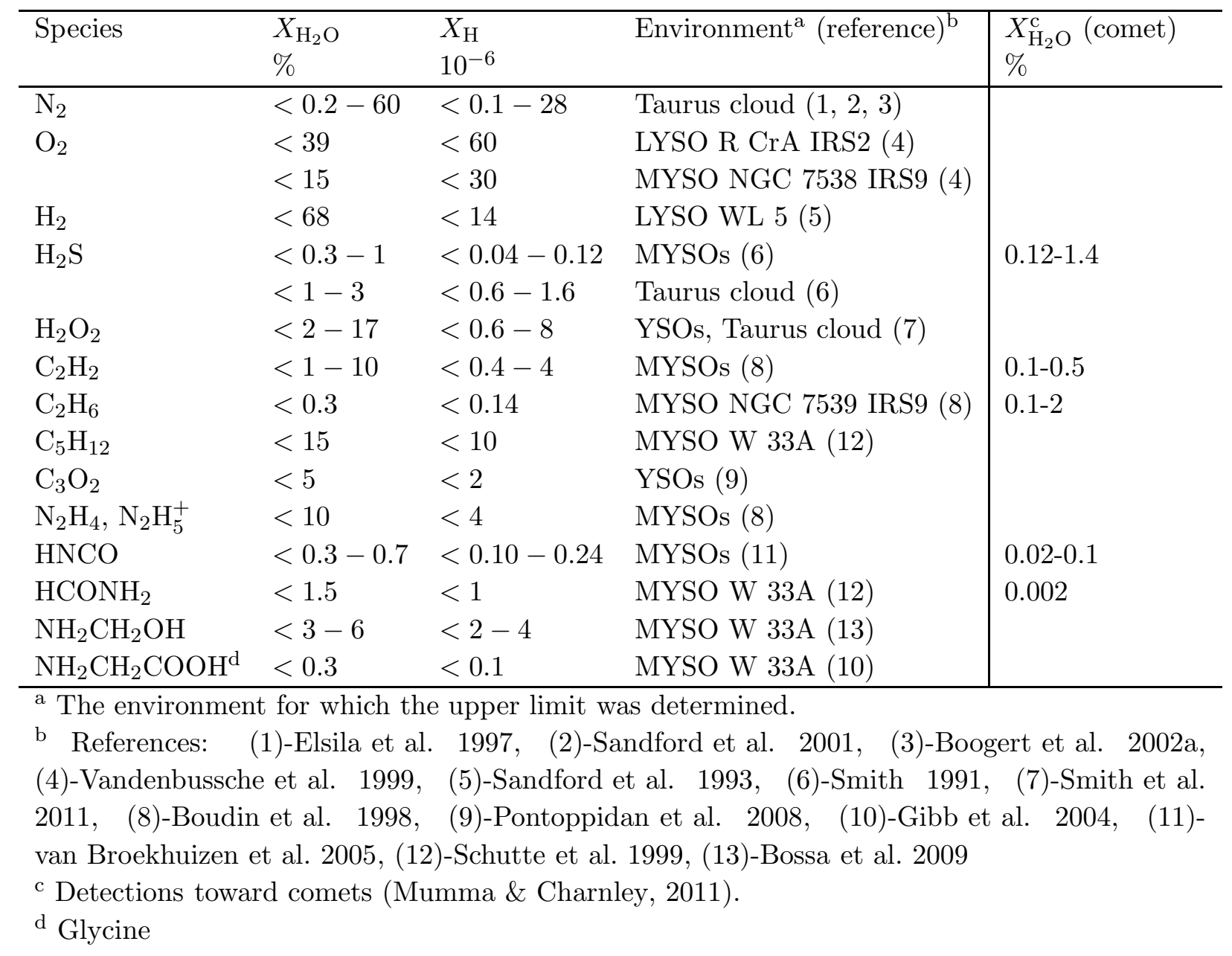

Most species in Table 3 were studied because of their expected production in the gas phase or on grain surfaces. $\mathrm{C}_{3} \mathrm{O}_{2}$ is a by-product of the energetic formation of $\mathrm{CO}_{2}$ (98.3). Species for which, to the best of our knowledge, no detections were claimed or upper limits were determined include the electronically stable "generation 0" species whose formation was studied in the laboratory and are listed by Theulé et al. (2013): $\mathrm{HCN}, \mathrm{CH}_{3} \mathrm{NH}_{2}, \mathrm{CH}_{2} \mathrm{NH}, \mathrm{NH}_{2} \mathrm{OH}, \mathrm{NH}_{2} \mathrm{CHO}, \mathrm{CH}_{3} \mathrm{CHO}$, and $\mathrm{HNCO}$. Other relevant species include $\mathrm{O}_{3}, \mathrm{HOCN}$, and $\mathrm{H}_{2} \mathrm{CO}_{3}$. Energetic processing and thermal reactions efficiently produce more complex species, but these are increasingly hard to detect because of a lack of distinct absorption features, as evidenced by the high upper limit of glycine listed in Table 3. Millimeter wave spectroscopy of gas phase species is orders of magnitude more sensitive, and must be used to determine their abundances after sublimation off the grains.

\subsection{Abundance Variations}

A recurring conclusion in the literature is that the ice abundances relative to $\mathrm{H}_{2} \mathrm{O}$ are to the first order similar over a wide range of environments. As sample sizes and uncertainties have improved, clear trends of variations by factors of 2-10 were discerned (Table 2). Most of these relate to CO depletion and subsequent 


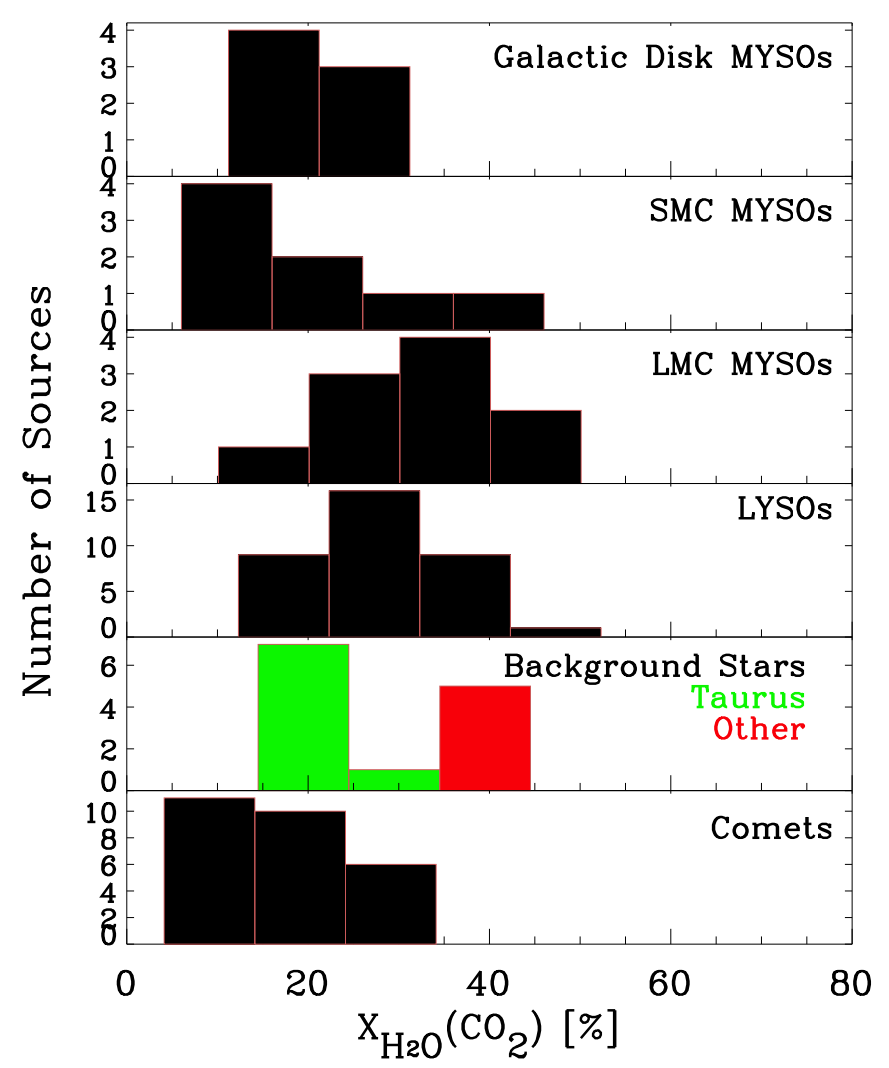

Figure 8: $\mathrm{CO}_{2}$ abundances relative to $\mathrm{H}_{2} \mathrm{O}$ in a range of environments. For the comets, the abundance represents the ratio of gas phase production rates (within the $\mathrm{H}_{2} \mathrm{O}$ sublimation radius at 2.7 AU; Ootsubo et al. 2012). For background stars, Taurus has distinctly lower abundances than other regions.

chemical reactions (88.1). A statistical study by Öberg et al. (2011) revealed that $X_{\mathrm{H}_{2} \mathrm{O}}(\mathrm{CO})$ and $X_{\mathrm{H}_{2} \mathrm{O}}\left(\mathrm{CO}_{2}\right)$ are factors of $\sim 3$ and 1.5 larger toward LYSOs and the quiescent regions of isolated cores compared to MYSOs and Taurus background stars. Within samples of MYSOs and LYSOs, $X_{\mathrm{H}_{2} \mathrm{O}}\left(\mathrm{CH}_{3} \mathrm{OH}\right)$ and $X_{\mathrm{H}_{2} \mathrm{O}}\left(\mathrm{OCN}^{-}\right)$ (4.64 $\mu \mathrm{m}$ component) vary by an order of magnitude. $\mathrm{CH}_{3} \mathrm{CH}_{2} \mathrm{OH}$ or $\mathrm{HCOOH}$ fall in this category too. In quiescent regions, the observed $X_{\mathrm{H}_{2} \mathrm{O}}\left(\mathrm{CH}_{3} \mathrm{OH}\right)$ varies from $<3 \%$ (Taurus) to $\sim 12 \%$ in some isolated dense cores.

The overview by Öberg et al. (2011) does not include extragalactic and GC ices, which are particularly well studied in $\mathrm{CO}_{2}$. Figure 8 shows a comparison of $X_{\mathrm{H}_{2} \mathrm{O}}\left(\mathrm{CO}_{2}\right)$ in all environments with accurate measurements. It highlights the systematic differences between environments, which are often rather small, but significant. MYSOs in the Galactic Disk have abundances that are typically a factor of 1.5-2 smaller compared to those in the LMC and compared to Galactic LYSOs. SMC MYSOs have low abundances when the $\mathrm{H}_{2} \mathrm{O}$ column is low, but converge to the LMC values at higher columns (Oliveira et al., 2013). For background stars, the distribution is doublepeaked. Low abundances $(\sim 20 \%)$ are observed toward seven stars behind Taurus (Whittet et al., 2009), while high abundances $(\sim 40 \%)$ are found for five lines of sight behind the Serpens (Knez et al., 2005) and Lupus (Boogert et al., 2013) clouds, and the IC 5146 (Whittet et al., 2009), L429-C, and L483 cores (Boogert et al., 2011). The discriminator appears to be the line of sight extinction: all but one of the Taurus sightlines have $A_{\mathrm{V}}<25 \mathrm{mag}$, while all but one of the other sightlines have $A_{\mathrm{V}}>25$ 
mag. It may thus be related to CO freeze out (88.1).

The $15.2 \mu \mathrm{m} \mathrm{CO} \mathrm{CO}_{2}$ band profiles vary between environments as well. The 5component fits applied to LYSOs and Galactic MYSOs (Pontoppidan et al., 2008) were also applied to MYSOs in the GC CMZ (An et al., 2011) and in the LMC (Seale et al., 2011). The GC MYSOs have twice as much $\mathrm{CH}_{3} \mathrm{OH}: \mathrm{CO}_{2}$ and $\mathrm{CO}: \mathrm{CO}_{2}$ ices relative to other environments (Fig. 9). LMC MYSOs, on the other hand, have much stronger pure $\mathrm{CO}_{2}$ components, indicating a larger degree of processing. Not shown here is the dominant $\mathrm{CO}_{2}: \mathrm{H}_{2} \mathrm{O}$ component, which is somewhat less abundant for the GC MYSOs and LMC MYSOs ( 55\%) compared to Galactic LYSOs $(\sim 70 \%)$. For the LMC sources, the sum of the pure $\mathrm{CO}_{2}$ and $\mathrm{CO}_{2}: \mathrm{H}_{2} \mathrm{O}$ ices is similar to the polar fraction toward LYSOs (Seale et al., 2011), suggesting that the pure $\mathrm{CO}_{2}$ ices are "tapped" from the polar ices upon heating. For GC MYSOs, the $\mathrm{CH}_{3} \mathrm{OH}: \mathrm{CO}_{2}$ component makes up for the difference with LYSOs. Finally, some LYSOs have lower $\left(\mathrm{CO}_{2}: \mathrm{H}_{2} \mathrm{O}\right) / \mathrm{CO}_{2}$ fractions and higher $X_{\mathrm{H}_{2} \mathrm{O}}\left(\mathrm{CO}_{2}\right)$, which is due to enhanced $\mathrm{CO}_{2}$ formation in CO-rich environments (e.g., source SVS 4-10 in Fig. (5))

Variations of ice abundances relative to $N_{\mathrm{H}}$ are evident too. The CO depletion in nearby quiescent clouds is incomplete (40-50\%; Whittet et al. 1985; Chiar et al. 1994) and the $\mathrm{H}_{2} \mathrm{O}$ abundance is also a factor of $\sim 2$ below the values seen toward embedded YSOs (Boogert et al., 2013). At higher densities $\left(10^{5}-10^{6} \mathrm{~cm}^{-3}\right)$ in cores and envelopes all ice abundances increase, but especially those of $\mathrm{CO}$ and chemically related species $\left(\mathrm{CH}_{3} \mathrm{OH}, \mathrm{CO}_{2}, \mathrm{OCN}^{-}\right.$; Pontoppidan et al. 2004).

\subsection{Isotopologues}

The ${ }^{13} \mathrm{CO}$ and ${ }^{13} \mathrm{CO}_{2}$ isotopologues were securely detected toward interstellar sources, and HDO tentatively. ${ }^{12} \mathrm{CO} /{ }^{13} \mathrm{CO}$ and ${ }^{12} \mathrm{CO}_{2} /{ }^{13} \mathrm{CO}_{2}$ ratios of $68-71$ (two sightlines; Boogert et al. 2002a; Pontoppidan et al. 2003) and 52-110 (thirteen sightlines; Boogert et al. 2000a) were derived, respectively. The ${ }^{12} \mathrm{CO}_{2} /{ }^{13} \mathrm{CO}_{2}$ ratios increase with Galactocentric radius, in accordance with the elemental isotopic ratios. HDO was tentatively detected at an abundance of $2-22 \%$ relative to $\mathrm{H}_{2} \mathrm{O}$ toward LYSOs with edge-on disks (Aikawa et al., 2012). Otherwise, low upper limits were derived: $<0.2-1 \%$ for intermediate mass and massive YSOs (Dartois et al., 2003), and $<0.5-2 \%$ for LYSOs (Parise et al., 2003). The relatively low deuterium fractionation of $\mathrm{H}_{2} \mathrm{O}$ is consistent with formation in an early cloud phase (Ceccarelli et al. 2014; (8.1). 

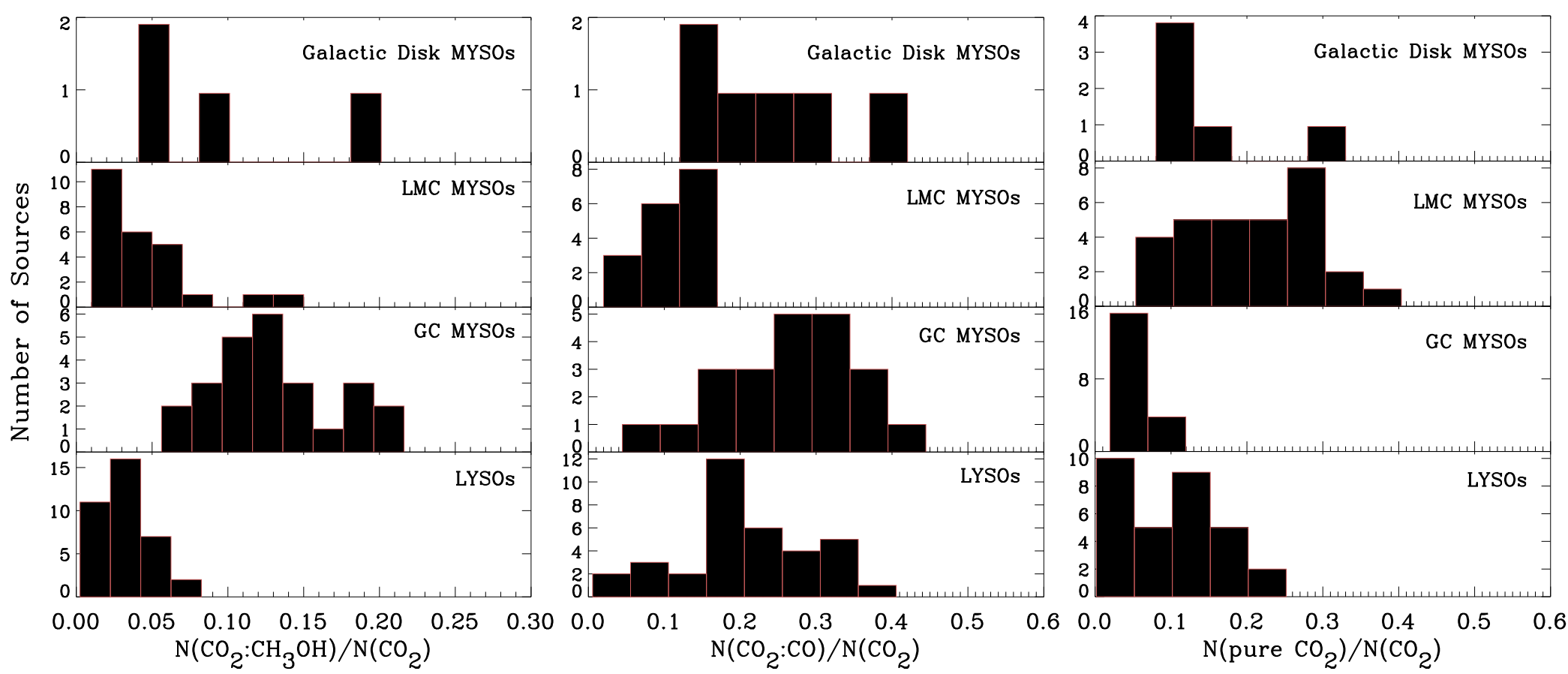

Figure 9: The $15.2 \mu \mathrm{m}$ band of $\mathrm{CO}_{2}$ shows distinct profile variations. Decompositions following Pontoppidan et al. (2008) show that the $\mathrm{CO}_{2}$ : $\mathrm{CH}_{3} \mathrm{OH}$ and $\mathrm{CO}_{2}$ : $\mathrm{CO}$ components are particularly strong toward the GC MYSOs. LYSOs show a particular lack of $\mathrm{CO}_{2}$ : $\mathrm{CH}_{3} \mathrm{OH}$, while $\mathrm{MYSO}$ in the LMC show an enhancement in pure $\mathrm{CO}_{2}$. Not shown is the $\mathrm{CO}_{2}: \mathrm{H}_{2} \mathrm{O}$ component, for which median values are quoted in 86.3 . 


\subsection{Interstellar Versus Cometary Ice Abundances}

Nearly all securely, likely, and possibly detected ice species outside the solar system are also detected toward comets (Table 2). Cometary ices are indirectly traced after outgassing and ions have recombined to their neutral counterparts. Interstellar $\mathrm{NH}_{4}^{+}$should thus be compared to cometary $\mathrm{NH}_{3}, \mathrm{OCN}^{-}$to $\mathrm{HNCO}$, and $\mathrm{HCOO}^{-}$to $\mathrm{HCOOH}$. Overall, the median abundances of all $\mathrm{C}$ - and $\mathrm{N}$-bearing species relative to $\mathrm{H}_{2} \mathrm{O}$ in comets are below those of LYSOs. For $\mathrm{CH}_{4}, \mathrm{CH}_{3} \mathrm{OH}$, and CO, the distributions overlap, however (Öberg et al., 2011). Using the measurements in comets from Ootsubo et al. (2012), a similar conclusion can be drawn for $\mathrm{CO}_{2}$ ices (Fig. 8): the median abundance is well below that of LYSOs (the upper quartile of comets is similar to the lower quartile of LYSOs), but there is some overlap. For $\mathrm{CH}_{3} \mathrm{OH}$, it appears that the overlap is caused by the class of organic-enriched comets, which has members from both the Oort Cloud and the Kuiper Belt, and has abundances similar to the median of LYSOs (Mumma \& Charnley, 2011). There is no overlap in the distribution of $\mathrm{NH}_{3}$ abundances, however. The discrepancy is even larger when the $\mathrm{NH}_{3}$ and $\mathrm{NH}_{4}^{+}$abundances toward LYSOs are added.

Because cometary ices are traced indirectly using gas phase rotational transitions, more sensitive abundance measurements can be made compared to ice measurements toward LYSOs. This is evident in Table 3, which shows that upper limits for $\mathrm{H}_{2} \mathrm{~S}$, $\mathrm{C}_{2} \mathrm{H}_{2}, \mathrm{C}_{2} \mathrm{H}_{6}$, and HNCO toward LYSOs are all above the detections for comets. Considering that $\mathrm{HNCO}$ in comets may directly trace $\mathrm{OCN}^{-}$in LYSOs, the trend of low cometary $\mathrm{C}$ and $\mathrm{N}$ abundances are confirmed. The median is well below that of LYSOs, but there is some overlap. Finally, a number of species were detected toward comets, and no measurements are currently available for LYSO ices: HCN (0.08$0.5 \%), \mathrm{S}_{2}(0.001-0.25 \%), \mathrm{HOCH}_{2} \mathrm{CH}_{2} \mathrm{OH}(0.25 \%), \mathrm{HCOOCH}_{3}(0.09 \%), \mathrm{HNC}(0.003-$ $0.05 \%), \mathrm{CH}_{3} \mathrm{CN}(0.008-0.04 \%), \mathrm{HC}_{3} \mathrm{~N}(0.003-0.07 \%)$, and $\mathrm{H}_{2} \mathrm{CS}(0.05 \%)$. In view of the uncertain identification of a number of interstellar ice features, in particular those in the 5-8 $\mu \mathrm{m}$ wavelength region, a search for these ices is warranted.

\subsection{Elemental Budget}

The budget of chemical elements constrains the identification of detected features and helps direct searches for new volatile and refractory species. The securely-, likely-, and possibly-detected ices do not nearly account for the abundance of the heavy elements $\mathrm{O}, \mathrm{C}, \mathrm{N}$, and $\mathrm{S}$ that is presumably available to be included in volatiles (i.e, excluding depletion in known refractory dust and gas phase volatiles). For O, $\sim 35 \%$ is "missing", for $\mathrm{C} \sim 30 \%$, for $\mathrm{N} 70-85 \%$, and for $\mathrm{S} \sim 95 \%$.

The total amount of the available $\mathrm{O}$ included in $\mathrm{H}_{2} \mathrm{O}, \mathrm{CO}$, and $\mathrm{CO}_{2}$ ices is similar in different quiescent clouds $(\sim 26 \%)$, even though the abundances of the individual species vary (Whittet et al., 2009). Öberg et al. (2011) find a similar median value for MYSOs, and a somewhat larger median value of $34 \%$ for LYSOs, with a maximum of $61 \%$ in regions of high $\mathrm{CO}$ freeze out. There is lingering uncertainty concerning putative additional carriers (Whittet, 2010). Solid $\mathrm{O}_{2}$ could account for up to $10 \%$ of O based on the limit in Vandenbussche et al. (1999), and the possibly identified species $\mathrm{HCOOH}$ for another few percent.

Of the $\mathrm{C}$ budget presumably available for inclusion in volatiles, 27 and $14 \%$ is 
taken up by known ices in LYSOs and MYSOs, respectively (Öberg et al., 2011). Maximum values of a factor of 2 larger are measured. Frozen PAH species are likely an important reservoir of (refractory) carbon in the ices, at $18 \%$ of the volatile budget, at least, using absolute band strength measurements (Hardegree-Ullman et al., 2014) of neutral pyrene, but this needs to be re-assessed when the degree of ionization of the frozen PAHs is constrained (Keane et al., 2001b; Bouwman et al., 2011).

$\mathrm{NH}_{3}, \mathrm{NH}_{4}^{+}$, and $\mathrm{OCN}^{-}$account for $\sim 11 \%$ of the available $\mathrm{N}$ (Öberg et al., 2011). It would be half of that if the identifications of $\mathrm{NH}_{4}^{+}$and $\mathrm{OCN}^{-}$are incorrect. In some sightlines, up to $30 \%$ could be included in the ices. It is plausible that much of the "missing" $\mathrm{N}$ is in the form of solid $\mathrm{N}_{2}$, but this is extremely difficult to confirm (\$6.2). The (uncertain) upper limits to the solid $\mathrm{N}_{2}$ abundance (Table 3) correspond to about $40 \%$ of the cosmic $\mathrm{N}$ budget.

Finally, OCS and, if its identification is confirmed, $\mathrm{SO}_{2}$, contribute only $4 \%$ to the available sulfur budget (Palumbo et al., 1997). The upper limit to $\mathrm{H}_{2} \mathrm{~S}$ (Table 3) would account for another percent.

\section{GRAIN SIZE AND MANTLE THICKNESS}

Mantle growth is expected to proceed at a rate independent of the grain size, although the smallest grains $(<0.002 \mu \mathrm{m})$ will be ice-less as they become too hot upon stochastic photon heating (Hollenbach et al., 2009). The elemental budget (86.6) gives upper limits to the mantle thickness. For $\mathrm{H}_{2} \mathrm{O}$-rich ices this is $0.005 \mu \mathrm{m}$ assuming an MRN grain radius $a$ distribution, at which point all $\mathrm{O}$ not included in gaseous $\mathrm{CO}$ and silicates is presumed to be incorporated into $\mathrm{H}_{2} \mathrm{O}$. Subsequent complete freeze-out of CO increases the mantle thickness by a further factor $\sim 2$. Such mantle growth can thus not significantly increase the sizes of the largest grains $(a>0.1 \mu \mathrm{m})$, but it can lead to a large fractional increase in the sizes of the abundant small grains that represent most of the surface area of the dust. Coagulation of the sticky icy grains will lead to further growth in dense molecular clouds (Ormel et al., 2009).

The observed ice features place some constraints on the grain size and mantle thickness. There is strong evidence for grains that are small compared to the wavelength $(a<<\lambda / 2 \pi)$ with comparatively thick ice mantles. The peak optical depth of the $\mathrm{H}_{2} \mathrm{O}$ stretch mode is generally centered at $3.0 \mu \mathrm{m}(a<<0.5 \mu \mathrm{m})$, and the profiles of the $\mathrm{CO}$ and $\mathrm{CO}_{2}$ stretch modes are single peaked. Thin mantled grains (core over mantle volume ratios larger than $\sim 0.2$; Tielens et al. 1991) would have doublepeaked profiles. In addition, spectrally resolved polarimetry of the $3.0 \mu \mathrm{m}$ band shows a shift of the polarization peak to longer wavelengths indicative of dichroic polarization (alignment of elongated grains by magnetic fields; Whittet 2011a; Andersson et al., this volume) by grains smaller than $\sim 0.1 \mu \mathrm{m}$; (Hagen et al., 1983; Hough et al., 1996). To test the presence of ices on grains much smaller than this, observations of the electronic transition of $\mathrm{H}_{2} \mathrm{O}$ at $0.14 \mu \mathrm{m}$ would be needed.

There is evidence for icy grains that are large compared to the wavelength as well, but with the exception of some clear-cut cases (e.g., abundant $\sim 0.8 \mu \mathrm{m}$ icy grains in an LYSO disk; 95.4 , Terada \& Tokunaga 2012a) it has encountered problems. Mie scattering calculations show that grain sizes of up to $\sim 0.5 \mu \mathrm{m}$ would be needed to explain the long wavelength wing of the $3.0 \mu \mathrm{m}$ ice band (\$4.1). Such large grains are, 
however, inconsistent with the spectropolarimetric measurements mentioned above. Also, in models satisfying all observational constraints (grain size distribution, elemental budget, ice band profiles) Smith et al. (1993) find that their observations of the $3.0 \mu \mathrm{m}$ profile in Taurus can only be explained if ice mantles are preferentially present on specific grain populations (e.g., on larger grains or on silicate grains).

Indirect evidence for a distinct population of much larger, micron-sized icy grains comes from diffuse scattered light observed toward dense cloud cores ("coreshine"; Andersen et al. 2014). Such grains were also suggested as an alternative explanation of the long-wavelength wing of the $4.67 \mu \mathrm{m}$ CO ice band (4.2, Dartois 2006). Large grains have been suggested as a source of the "missing" oxygen (46.6, Jenkins 2009), because for radii larger than a few micron, the infrared absorption profiles become too wide and shallow to be detected. In light of the small variations of the $3.0 \mu \mathrm{m}$ band profile (4.1), these suggestions need further study, including an assessment of the effects of grain coagulation on the band profiles.

\section{CONSTRAINTS ON ICE EVOLUTION MODELS}

The commonalities and differences in the ice abundances and band profiles observed over a wide range of environments provide strong constraints on models of ice formation and evolution. We do not review the models here, but rather summarize the observational constraints. Figure 10 illustrates the probable scenario.

\subsection{Accretion and Grain Surface Chemistry}

The observed ice abundances are generally in good agreement with models of accretion from the gas phase followed by grain surface chemistry. In particular, the efficiency of hydrogenation as a result of the mobility and tunneling capacity of $\mathrm{H}$ atoms (e.g., Tielens \& Hagen 1982) is confirmed by the observations. The dominance of $\mathrm{H}_{2} \mathrm{O}$ ice and mixtures with $\mathrm{H}_{2} \mathrm{O}$ in all Galactic and extragalactic environments points to a phase of molecule formation on cold grain surfaces at which the accreting gas is atomic $\mathrm{H}$ - and O-rich. This is a phase of relatively low densities $\left(n \geq 10^{3} \mathrm{~cm}^{-3}\right)$ early in the cloud evolution $\left(\mathrm{H} / \mathrm{H}_{2}\right.$ ratios decrease rapidly with density; Hollenbach et al. 1971). The ice formation threshold of $A_{\mathrm{V}}=1.6 \mathrm{mag}$ near local cloud edges is understood as the onset of rapid ice mantle growth after a monolayer has formed and the effect of photodesorption decreases with increasing extinction by dust into the cloud $\left(A_{\mathrm{V}} \propto \ln \left(G_{0} / n\right)\right.$; Hollenbach et al. 2009). This phase also includes the formation of $\mathrm{CH}_{4}, \mathrm{CO}_{2}$ (polar component, likely via $\mathrm{CO}+\mathrm{OH}$; Ioppolo et al. 2011), and $\mathrm{NH}_{3}$ which are all intimately mixed with $\mathrm{H}_{2} \mathrm{O}$. Variations in $\mathrm{CO}_{2} / \mathrm{H}_{2} \mathrm{O}$ ratios in this early phase reflect variations of the $\mathrm{CO}$ accretion rates. On one hand, if the gas phase formation of $\mathrm{CO}$ is incomplete, e.g., at low extinctions, more $\mathrm{O}$ can be included in $\mathrm{H}_{2} \mathrm{O}$ (Whittet et al., 2009; Öberg et al., 2011). It may explain the possibly $\mathrm{CO}_{2}$-free region in the SMC (Oliveira et al., 2013) and $\mathrm{CH}_{4}{ }^{-}$

rich region in Galactic cloud edges (Öberg et al., 2011). On the other hand, CO accretion rates increase at lower temperatures and at higher densities, leading to enhanced formation of $\mathrm{CO}_{2}$ (up to a factor of 2), still well mixed with the co-formed $\mathrm{H}_{2} \mathrm{O}$ species. This phase is $\mathrm{CH}_{3} \mathrm{OH}$-poor and has a relatively low $\mathrm{CO}$ freeze out $(<50 \%)$. At the same time, the presence of the $6.85 \mu \mathrm{m}$ feature and some absorption 
underlying the $6.0 \mu \mathrm{m}$ band (Fig. 6) show that these early ices are already quite complex. They are formed on a short time scale $\left(10^{9} / n \sim 10^{5}\right.$ yr at $n=10^{4} \mathrm{~cm}^{-3}$; e.g., Hollenbach et al. 2009) compared to the cloud life time $\left(\sim 10^{7} \mathrm{yr}\right)$ and compared to the formation time of new species by interstellar CRs or CR-induced UV fields ( 98.3$)$.

Strong enhancements (up to a factor of 10) of the abundances of $\mathrm{CH}_{3} \mathrm{OH}, \mathrm{OCN}^{-}$, the carriers of the 7.24 and $7.41 \mu \mathrm{m}$ features $\left(\mathrm{CH}_{3} \mathrm{CH}_{2} \mathrm{OH}\right.$ or $\mathrm{HCOOH}$ and $\mathrm{CH}_{3} \mathrm{CHO}$ or $\mathrm{HCOO}^{-}$), and the apolar components of the $\mathrm{CO}$ and $\mathrm{CO}_{2}$ ices highlight the subsequent phase of ice formation. It is the episode of "catastrophic" CO freeze out at low temperatures and high densities $\left(n \geq 10^{5} \mathrm{~cm}^{-3}\right)$, when the gas phase $\mathrm{H} / \mathrm{CO}$ ratios are drastically enhanced, even though the $\mathrm{H} / \mathrm{H}_{2}$ ratios are reduced. This then leads to $\mathrm{H}_{2} \mathrm{CO}$ and $\mathrm{CH}_{3} \mathrm{OH}$ on a short time scale of $\sim$ few $\times 10^{4}$ years, as demonstrated in Monte Carlo simulations (Cuppen et al., 2009), and likely to a host of other CO-derived species, including HNCO (Fedoseev et al., 2015) and $\mathrm{CO}_{2}$ (in the apolar component). The best observational evidence of this phase comes from the measurements of ice abundance gradients in dense cores and Class 0 LYSOs (Pontoppidan et al., 2004; Pontoppidan, 2006). The quiescent regions in the Taurus cloud have not entered this phase yet (Fig. 8), in contrast to a subset of dense cores, prestellar cores, and LYSOs (e.g., SVS 4-10 in Fig. 5 has a $\mathrm{CO}_{2} / \mathrm{H}_{2} \mathrm{O}$ ratio of $50 \%$ and a strong CO: $\mathrm{CO}_{2}$ component; Pontoppidan et al. 2003). Clearly, a complex mixture of ices is formed before stars form. Apparently, the GC MYSOs (prominent apolar $\mathrm{CO}_{2}$ and $\mathrm{CH}_{3} \mathrm{OH}: \mathrm{CO}_{2}$ components) have gone through this phase, but the LMC MYSOs have not (weak apolar $\mathrm{CO}_{2}$ and $\mathrm{CH}_{3} \mathrm{OH}: \mathrm{CO}_{2}$ components and low solid CO abundances). We speculate that high and low metallicities in these distinct environments, respectively, play a role. High metallicities reduce the $\mathrm{H} / \mathrm{CO}$ ratios and increase extinction and thus CO freeze out. 


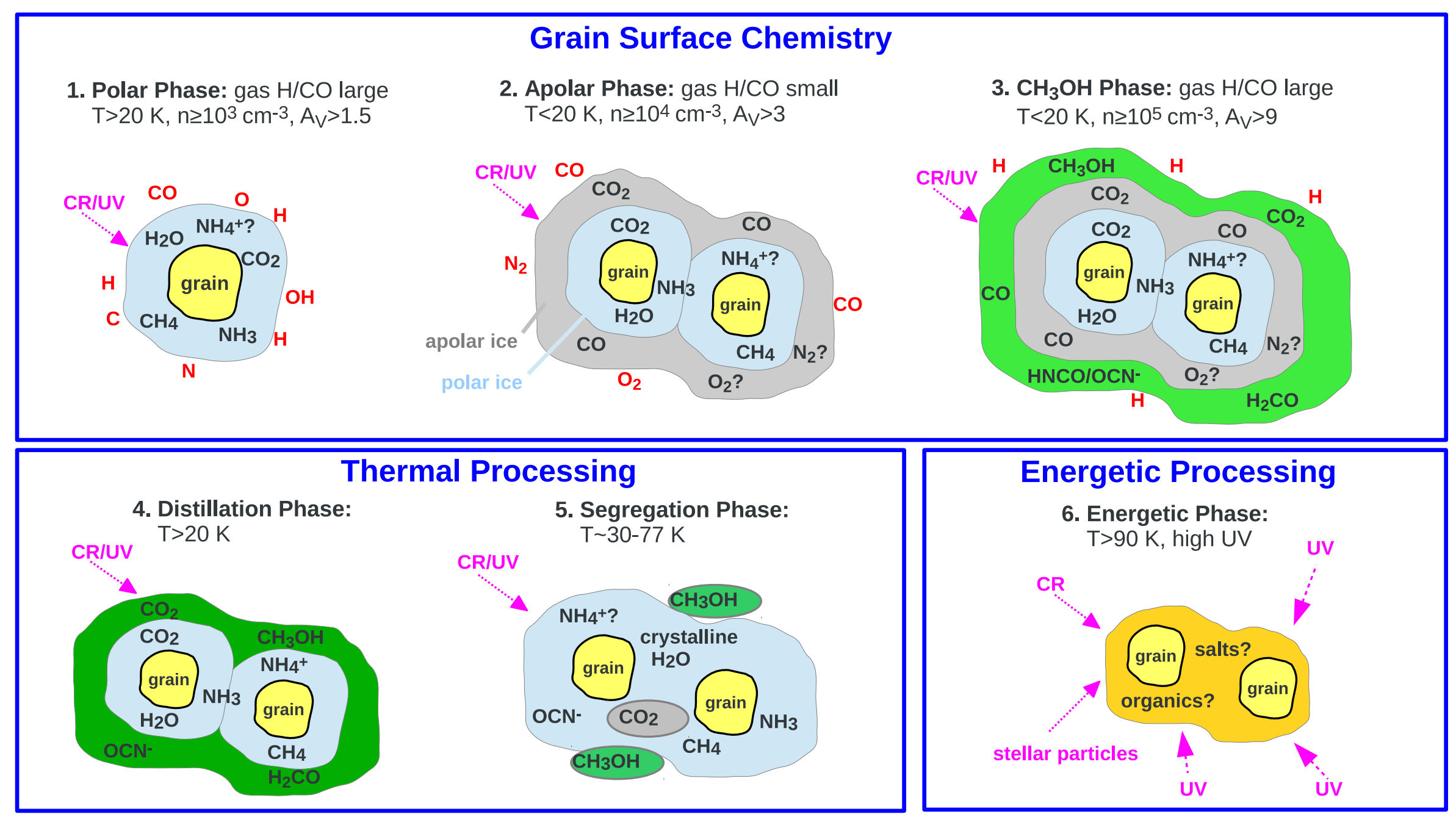

Figure 10: Schematic view of the observationally constrained composition of icy grain mantles, highlighting the physical and chemical processes at play. Six phases are shown, which could represent progressive evolutionary phases in quiescent clouds (phases 1-3) and YSO envelopes and disks (phases 3-6). Gas phase species participating in grain surface reactions are indicated in red. In phases 1-5, the ices experience CR and secondary UV fields that only minimally affect the main ice species. As the ice temperature rises (phases 4 and 5 ), thermal reactions or reactions involving radicals may proceed. Stage 6 is least observationally constrained. The relative grain and mantle sizes in the first 5 stages are realistic (maximum mantle thickness of $50 \AA$ and minimal grain core sizes of $20 \AA$ ). After the initial ice layers are formed (phase 1), grain coagulation accelerates. 


\subsection{Thermal Processing}

Observations show that after the formation of the bulk of the ices through accretion and grain surface chemistry, the evolution of the ices in the circumstellar envelopes and disks of YSOs is dominated by thermal processes (\$4). In summary, sublimation of apolar ices occurs at temperatures of $\sim 20 \mathrm{~K}$. As CO is "distilled" out of the $\mathrm{CO}_{2}$ ices, this develops the 15.11 and $15.26 \mu \mathrm{m}$ absorption peaks of pure $\mathrm{CO}_{2}$. Segregation of $\mathrm{CO}_{2}$ from $\mathrm{H}_{2} \mathrm{O}$-rich ices occurs at higher temperatures, starting at $30 \mathrm{~K}$ for the surface layers and thin mantles (Öberg et al., 2009b), and up to $\sim 77 \mathrm{~K}$ for thick mantles (Boogert et al., 2000a) at which the $\mathrm{H}_{2} \mathrm{O}$ crystallization process starts as well. This is observed by a long-wavelength shift and narrowing of the $3.0 \mu \mathrm{m}$ band, and again by the development of the 15.11 and $15.26 \mu \mathrm{m}$ absorption peaks. The "pure" $\mathrm{CO}_{2}$ will sublimate at temperatures in the range of $\sim 45 \mathrm{~K}$ for "distilled ices", up to the $\mathrm{H}_{2} \mathrm{O}$ sublimation temperature of $\sim 90 \mathrm{~K}$ for segregated ices, depending on the mantle thickness (Fayolle et al., 2011). Sublimation of the polar, $\mathrm{H}_{2} \mathrm{O}$-rich ices is traced by increased gas/solid state column density ratios (Boonman et al., 2003). The strength of the $\mathrm{C} 4$ component of the $6.85 \mu \mathrm{m}$ feature is apparently enhanced at temperatures above the $\mathrm{H}_{2} \mathrm{O}$ sublimation temperature (Boogert et al., 2008).

The sublimation, segregation, and crystallization temperatures quoted in this review apply to the long time scales in the interstellar environments. At any temperature, the probability that the energy barrier for these processes is surmounted is set by the Boltzmann distribution. This translates to time scales of $\nu_{0}^{-1} \mathrm{e}^{E_{\mathrm{barrier}} / T}$ (Tielens \& Allamandola, 1987b). For example, for $\mathrm{H}_{2} \mathrm{O}$, with an energy barrier of $E_{\text {barrier }}=5500 \mathrm{~K}$ and a frequency $\nu_{0}=5 \times 10^{-13} \mathrm{~s}^{-1}$ (O-H bend vibration mode), an observed sublimation temperature of $180 \mathrm{~K}$ at a laboratory time scale of minutes corresponds to $90 \mathrm{~K}$ at space time scales of 10,000 years. These arguments were used to link the range of pure $\mathrm{CO}_{2}$ peak strengths in a sample of MYSOs to a time scale of $\sim 3 \times 10^{4}$ yrs needed to heat most of their envelopes to temperatures of $\sim 77 \mathrm{~K}$ (Boogert et al., 2000a). Models show that envelope heating and dispersal occur at the same time (van der Tak et al., 2000). For low mass envelopes, dynamical time scales were coupled to temperatures, which is critical to locate the various processes (Pontoppidan et al., 2008). For a $1 M_{\odot}, 3 L_{\odot}$ star, this translates to an apolar ice sublimation radius of $1000 \mathrm{AU}$, a crystallization and segregation region between 100 and $130 \mathrm{AU}$, and polar ice sublimation within $100 \mathrm{AU}$. In circumstellar disks surrounding similar stars, the observed ice crystallization (\$5.4) is also thought to happen at radii $\geq 100 \mathrm{AU}$, but only in their super-heated optically thin surfaces (Chiang et al., 2001).

For objects of very low luminosity, envelope heating is insufficient to cause crystallization and segregation (Kim et al., 2012). The double peaked $\mathrm{CO}_{2}$ profile is detected toward $<0.1 \mathrm{~L} \odot$ YSOs, however. It is suggested that the apolar $\mathrm{CO}_{2}$ component is responsible for that, in combination with heating spikes caused by episodic accretion events. During long intervals between the accretion events, $\mathrm{CO}_{2}$ is formed in the $\mathrm{CO}$-rich ice by reactions with $\mathrm{OH}$. The heating event then sublimates the $\mathrm{CO}$, and leaves pure $\mathrm{CO}_{2}$ behind. In a separate study, a $\mathrm{CO}_{2}$ bending mode consisting of nearly pure $\mathrm{CO}_{2}$ is found toward the $1 L_{\odot}$ YSO HOPS 68 (Fig. 5, Poteet et al. 2013). This may also be interpreted as a result of episodic heating, but in this case the event is much stronger (up to $100 L_{\odot}$, as in FU Ori objects) and all ices are 
sublimated and then recondensed in the order of their condensation temperature. Fortuitous timing and line of sight geometry inside a flattened envelope are required to trace this particular ice component.

The common process of ice heating most likely affects the chemistry as well, although from an observational point of view there is little direct evidence for this. A candidate feature produced upon heating is the "C5" component (Fig. 6). We speculate that it might be formed when the radicals, created by energetic processes over the cloud and envelope life time, become more mobile and react at higher temperatures (Garrod et al., 2008; Vasyunina et al., 2014). Finally, purely thermal reactions, without the help of energetic processes, may well be quite common (Schutte et al., 1993; Bossa et al., 2009; Theulé et al., 2013). The formation of $\mathrm{NH}_{4}^{+}$and $\mathrm{OCN}^{-}$, (proposed) carriers of the 6.85 and $4.62 \mu \mathrm{m}$ features, proceeds in fact by an acidbase reaction of $\mathrm{NH}_{3}$ with $\mathrm{HNCO}$ at temperatures as low as $15 \mathrm{~K}$ (Raunier et al., 2004b; van Broekhuizen et al., 2004). This is consistent with $\mathrm{NH}_{4}^{+}$being the carrier of the $6.85 \mu \mathrm{m}$ feature, as it is observed in quiescent sightlines. HNCO cannot be the only acid involved in this reaction, however, as the $\mathrm{OCN}^{-}$abundance is an order of magnitude below that of $\mathrm{NH}_{4}^{+}$.

\subsection{Energetic Processing}

Historically, it has long been held that the energetic processing of ices in space plays a key role in the production of new ice species and specifically in the transformation from simple to more complex components (e.g., Greenberg 1973). Ices in dense clouds experience $\mathrm{CR}$ fluxes that are represented by $1-\mathrm{MeV}$ protons with a flux of 1 proton $\mathrm{cm}^{-2} \mathrm{~s}^{-1}$ (Moore et al. 2001 and references therein). The interstellar UV field of $\sim 10^{9} G_{0} \mathrm{eV} \mathrm{cm}^{-2} \mathrm{~s}^{-1}$ (with $G_{0}=1$ in the local ISM) rapidly declines with depth into the cloud, regulating the on-set of ice formation (e.g., Hollenbach et al. 2009). Within clouds, the fluxes of UV photons are much lower, generated by CR destruction of $\mathrm{H}_{2}$ and corresponding to $\sim 10^{4} \mathrm{eV} \mathrm{cm}^{-2} \mathrm{~s}^{-1}$. In regions of star formation these fluxes may be strongly enhanced in both space and in time.

A vast literature of laboratory studies of energetic processing exists, which has been particularly successful in solar system studies (e.g., Paranicas et al. 2009). In contrast to grain surface chemistry and thermal processing, the significance of energetic processing of ices outside the solar system has not yet been unambiguously demonstrated, however. Observational tests of energetic processing must address the similarities and differences between laboratory and observed interstellar abundances, as well as the spectral profiles of the produced species of interest and all subsequent chemical (by-)products.

Many ice absorption features have been studied in the context of energetic processing, but most attention was paid to those of $\mathrm{CO}_{2}$, as well as those at 4.62 and $6.85 \mu \mathrm{m} . \mathrm{CO}_{2}$ is formed readily by the energetic processing of most carbon-bearing molecules in the presence of $\mathrm{H}_{2} \mathrm{O}$, including pure $\mathrm{H}_{2} \mathrm{O}$ ice mantles on carbon grains (Mennella et al., 2004). Ioppolo et al. (2013) have explored this to the full extent by fitting the $15.2 \mu \mathrm{m} \mathrm{CO} \mathrm{CO}_{2}$ bending mode toward MYSOs, LYSOs, and dense clouds using only $\mathrm{CO}_{2}$ produced by processing with Galactic CRs. Comparing these results to the empirical decomposition (Fig. 5), the broad polar component is fitted with $\mathrm{CO}_{2}$ produced from irradiated $\mathrm{H}_{2} \mathrm{O}$ on carbon grains, the shoulder from irradiated 
$\mathrm{CH}_{3} \mathrm{OH}$, the apolar components from irradiated $\mathrm{CO}$ and $\mathrm{CO}: \mathrm{N}_{2}$ ices, and the pure $\mathrm{CO}_{2}$ component by heating of these ices. At the observed abundances of $\mathrm{CO}, \mathrm{H}_{2} \mathrm{O}$, and $\mathrm{CH}_{3} \mathrm{OH}$, the $\mathrm{CO}_{2}$ profiles and abundances are reproduced after $\sim 10^{7} \mathrm{yr}$ for most targets. Similarly long time scales were found for the production of $\mathrm{CO}_{2}$ by the CR-generated UV field (see above) in dense clouds (Whittet et al., 1998). Thus, overall, after the initial rapid formation of $\mathrm{CO}_{2}$ and $\mathrm{H}_{2} \mathrm{O}$ by grain-surface reactions (in about $10^{5} \mathrm{yr}$; see 88.1 ), additional $\mathrm{CO}_{2}$ may be formed by particle bombardment during the millions of years in YSO environments, or on shorter time scales for regions exposed to stronger UV and particle fields. Not all implications of this model have been fully investigated, however. Most importantly, the effect of a suite of by-products, in particular carbon-chain oxides $\left(\mathrm{C}_{3} \mathrm{O}, \mathrm{C}_{3} \mathrm{O}_{2}\right.$; Gerakines et al. 1996; Gerakines \& Moore 2001a; Palumbo et al. 2008) on other spectral regions has not yet been fully assessed. Also, no distinct enhancements of $\mathrm{CO}_{2}$ abundances were observed toward YSOs (Fig. 8), although the situation is complicated by the susceptibility of $\mathrm{CO}_{2}$ itself to destruction by irradiation.

The $4.62 \mu \mathrm{m} \mathrm{XCN}$ feature was seen for a long time as an indicator of energetic processing, as laboratory UV photolysis and ion bombardment experiments on mixed $\mathrm{CO}$ and $\mathrm{NH}_{3}$ ices successfully reproduced this absorption feature with $\mathrm{OCN}^{-}$(96.1). Similar experiments claimed an identification of the $6.85 \mu \mathrm{m}$ feature with $\mathrm{NH}_{4}^{+}$(Schutte \& Khanna, 2003) and $\mathrm{H}_{2} \mathrm{NCONH}_{2}$ (urea; Raunier et al. 2004a). Both features can also be reproduced through purely thermal acid-base reactions in ices, however and in many sightlines this is the more likely process ( $₫ 8.2)$.

\subsection{Connection with Cometary Ices}

The survival of the protostellar envelope ices upon accretion into the protoplanetary disk is an open question. Depending on the radius to the central star, full or partial destruction of ices and subsequent reformation may have occurred (Mumma \& Charnley 2011, and references therein). The Stardust mission revealed that refractory dust may have formed close to the early Sun, moving radially outward, accumulating ices along the way and thus diluting any presolar components in the outer, comet formation zones (Brownlee, 2014). Ice observations provide constraints to this discussion, but have not been conclusive. There are distinct differences between the composition of cometary and LYSO envelope ices ( $(6.5)$. C- and N-bearing species in comets are under-abundant on average. For $\mathrm{C}$, the abundance distributions do overlap, which may imply that the early solar system resembled an LYSO with less C in the ices (Öberg et al., 2011). Low cometary $\mathrm{CO}$ and $\mathrm{CO}_{2}$ may also reflect sublimation of the most volatile, apolar components upon accretion from the envelope to the protoplanetary disk (Visser et al., 2009). Neither argument holds for N, however: the $\mathrm{NH}_{3}$ abundance distributions do not overlap and $\mathrm{NH}_{3}$ is only present in the polar (least volatile) ices. Direct observations of ices in the disk mid-plane, where comets are formed, are not possible. Ices in the disk surface may be linked to the midplane by turbulence, however. High spatial resolution observations have shown that these ices experience thermal processing (\$5.4). Further observations are needed to search for evidence of energetic processing, but chemical models including vertical disk turbulence have indicated that the ice composition throughout the disk may be significantly affected by energetic processes in the disk surface (Ciesla \& Sandford, 
2012). Radial transport might lead to such effects as well.

\section{SUMMARY POINTS}

Infrared observations have revealed the icy Universe (Fig. 10):

1. $\mathrm{H}_{2} \mathrm{O}$-based ices trace dense clouds $\left(n \geq 10^{3} \mathrm{~cm}^{-3}\right)$ at depths corresponding to $A_{\mathrm{V}} \geq 1.6$ and temperatures below $90 \mathrm{~K}$. These are mixed ices, containing at least $\mathrm{CO}_{2}, \mathrm{NH}_{3}, \mathrm{CH}_{4}$, and the carrier of the $6.85 \mu \mathrm{m}$ feature $\left(\mathrm{NH}_{4}^{+}\right.$?).

2. CO freeze-out occurs deeper in dense clouds $\left(A_{\mathrm{V}} \geq 3\right)$, forming an apolar ice layer, and it does so "catastrophically" at $A_{\mathrm{V}} \geq 9$ and $n \geq 10^{5} \mathrm{~cm}^{-3}$, initiating a CO-based chemistry on the ices forming a third, $\mathrm{CH}_{3} \mathrm{OH}-\mathrm{rich}$, layer including also $\mathrm{H}_{2} \mathrm{CO}$, newly formed $\mathrm{CO}_{2}$, and possibly $\mathrm{HNCO}$.

3. Heating of the ices is commonly observed and the spectroscopic signatures provide diagnostics of the thermal history of dense environments. Crystallization signatures in the 3.0, 45, and $63 \mu \mathrm{m} \mathrm{H}_{2} \mathrm{O}$ bands, and the position of the $6.85 \mu \mathrm{m}$ feature trace temperatures $\geq 77 \mathrm{~K}$. The double-peaked $15.2 \mu \mathrm{m} \mathrm{CO}_{2}$ band traces a combination of such high temperatures ("segregation") and temperatures $\geq 20 \mathrm{~K}$ as a result of $\mathrm{CO}$ sublimation ("distillation"). The ratio of the apolar/polar components of the $4.67 \mu \mathrm{m} \mathrm{CO}$ band itself traces the latter conditions as well.

4. Most observed ice features are consistent with molecule formation on cold grain surfaces. The observed ice heating may well induce chemical reactions, possibly involving radicals created by energetic processes over long time scales. The observational evidence for this, possibly features in the 5-7 $\mu \mathrm{m}$ region, is weak, however.

5. Variations of the $\mathrm{CO}_{2} / \mathrm{H}_{2} \mathrm{O}$ column density ratios and $\mathrm{CO}_{2}$ ice composition in local versus GC and extragalactic environments likely reflect metallicity differences.

6. Median abundances of $\mathrm{C}$ and N-bearing cometary ices are below those of the envelopes of LYSOs. The abundance distributions do overlap, however, and inheritance of cometary ices from the ISM and envelope phases can thus not be excluded.

7. Laboratory spectroscopy has been crucial in the identification and in the determination of the chemical and physical history of the observed ice features.

\section{FUTURE ISSUES}

1. Recent high spatial resolution observations of circumstellar disks have shown that the ice composition and processing history can be traced on scales relevant for planet and comet formation, both spectroscopically (e.g., Schegerer \& Wolf 2010; Terada \& Tokunaga 2012a) and using scattered light imaging (e.g., Honda et al. 2009; Debes et al. 2008). Future high angular resolution ground (Thirty Meter Telescope, Extremely Large Telescope) and space-based (James Webb Space 
Telescope; JWST) infrared facilities thus promise great advances of ices studies at these scales.

2. The same new facilities will enable ice studies in extreme environments (strong radiation fields, high or low metallicities), such as Galactic and extragalactic MYSOs at larger distances and in crowded fields. Observed feature profile and depth variations may further constrain identifications as well as molecule formation pathways, including the much sought after evidence for energetic processing.

3. The increased sensitivity of new facilities will drastically enhance studies of quiescent clouds at very low and high extinctions, which are needed to study the ice formation process and its interplay with the grain growth process.

4. The continuous 1-29 $\mu \mathrm{m}$ wavelength coverage provided by JWST at spectral resolving powers $R$ of up to 3000 will provide strong constraints on the identification of new ice species. Supporting laboratory experiments are essential for this.

5. New instruments on platforms such as the Stratospheric Observatory for Infrared Astronomy (SOFIA) and the Space Infrared Telescope for Cosmology and Astrophysics (SPICA) are needed to give access to the ice lattice modes $(>25 \mu \mathrm{m})$, which are particularly powerful tracers of the ice composition and thermal history. As they appear in emission, the ices may be mapped.

6. The good understanding of the main ice bands allows them to be used as tools in the characterization of new sources that will be found with new infrared facilities.

\section{RELATED RESOURCES}

Infrared transmission spectra and optical constants of astrophysically relevant pure ices and mixtures may be found at the following web sites:

- Astrophysical and Astrochemistry Laboratory at NASA Ames Research Center:

http: //astrochem.org/db.php

- Experimental Astrophysics Laboratory at Catania Astrophysical Observatory: http://www.ct.astro.it/lasp/

- Sackler Laboratory for Astrophysics (Leiden): http://www. laboratory-astrophysics.eu/

- Cosmic Ice Laboratory at NASA Goddard Space Flight Center: http://science.gsfc.nasa.gov/691/cosmicice/

- Grenoble Astrophysics and Planetology Solid Spectroscopy and Thermodynamics database (GhoSST):

http://ghosst.osug.fr

- Jena - St.Petersburg Database of Optical Constants (mostly references to laboratory spectra):

http://www. astro.uni-jena.de/Laboratory/Database/jpdoc

- Virtual Atomic and Molecular Data Centre (VAMDC):

http://portal.vamdc.org 
Computer programs for calculating the effect of grain shape and size on ice absorption band profiles can be found in Bohren \& Huffman (1983) and are also made available by B. T. Draine at

http://www . astro.princeton.edu/ draine/scattering.html

\section{ACKNOWLEDGMENTS}

We thank Deokkeun An, Jean Chiar, Sergio Ioppolo, Karin Öberg, Joana Oliveira, Laurent Pagani, Elisabetta Palumbo, Klaus Pontoppidan, Charles Poteet, Gianni Strazzulla, Xander Tielens, and the reviewer Ewine van Dishoeck for discussions and comments. We also thank Deokkeun An, Jean Chiar, Emmanuel Dartois, Leslie Looney, Joana Oliveira, Klaus Pontoppidan, Charles Poteet, Anna Sajina, Jonathan Seale, and Hiroshi Terada for kindly providing published data in electronic form. A.C.A.B. thanks encouragement from Mahsan, Cyrus, and Jasper to work on this review in a time of moves and job uncertainty.

\section{References}

Adams FC. 2010. ARAA 48:47

Aikawa Y, Kamuro D, Sakon I, et al. 2012. A\&A 538:57

Allamandola LJ, Sandford SA, Tielens AGGM, Herbst TM. 1992. ApJ 399:134

An D, Ramírez SV, Sellgren K, et al. 2011. ApJ 736:133

Andersen M, Thi W-F, Steinacker J, Tothill N. 2014. A\&A 568:L3

Baratta GA, Palumbo ME. 1998. Journal of the Optical Society of America A 15:3076

Bergin EA, Melnick GJ, Gerakines PA, Neufeld DA, Whittet DCB. 2005. ApJL 627:L33

Bergin EA, Tafalla M. 2007. ARAA 45:339

Bernstein MP, Sandford SA, Allamandola LJ, Chang S, Scharberg MA. 1995. ApJ 454:327

Bisschop SE, Jørgensen JK, van Dishoeck EF, de Wachter EBM. 2007. A\&A 465:913

Blake GA, Sutton EC, Masson CR, Phillips TG. 1987. ApJ 315:621

Bohren CF, Huffman DR. 1983. Absorption and Scattering of Light by Small Particles. John Wiley \& Sons, New York

Boogert ACA, Schutte WA, Tielens AGGM, et al. 1996. A\&A 315:L377

Boogert ACA, Schutte WA, Helmich FP, Tielens AGGM, Wooden DH. 1997. A\&A $317: 929$

Boogert ACA, Ehrenfreund P, Gerakines PA, et al. 2000a. A\&A 353:349

Boogert ACA, Tielens AGGM, Ceccarelli C, et al. 2000b. A\&A 360:683

Boogert ACA, Blake GA, Tielens AGGM. 2002a. ApJ 577:271

Boogert ACA, Hogerheijde MR, Blake GA. 2002b. ApJ 568:761

Boogert ACA, Hogerheijde MR, Ceccarelli C, et al. 2002c. ApJ 570:708

Boogert ACA, Blake GA, Öberg K. 2004a. ApJ 615:344 
Boogert ACA, Ehrenfreund P. 2004b. In: Astrophysics of Dust, ed. AN Witt, GC Clayton, BT Draine, ASP Conf. Ser. 309, p. 547

Boogert ACA, Pontoppidan KM, Knez C, et al. 2008. ApJ 678:985

Boogert ACA, Huard TL, Cook AM, et al. 2011. ApJ 729:92

Boogert ACA, Chiar JE, Knez C, et al. 2013. ApJ 777:73

Boonman AMS, Doty SD, van Dishoeck EF, et al. 2003. A\&A 406:937

Bossa JB, Theule P, Duvernay F, Chiavassa T. 2009. ApJ 707:1524

Bottinelli S, Boogert ACA, Bouwman J, et al. 2010. ApJ 718:1100

Boudin N, Schutte WA, Greenberg JM. 1998. A\&A 331:749

Bouwman J, Mattioda AL, Linnartz H, Allamandola LJ. 2011. A\&A 525:93

Brooke TY, Sellgren K, Geballe TR. 1999. ApJ 517:883

Brownlee D. 2014. Annual Review of Earth and Planetary Sciences 42:179

Brunetto R, Barucci MA, Dotto E, Strazzulla G. 2006. ApJ 644:646

Ceccarelli C, Caselli P., Bockelée-Morvan D, et al. 2014. In Protostars and Planets VI, University of Arizona Press, ed. H Beuther, R Klessen, C Dullemond, Th Henning, p. 859-882

Chiang EI, Joung MK, Creech-Eakman MJ, et al. 2001. ApJ 547:1077

Chiar JE, Adamson AJ, Kerr TH, Whittet DCB. 1994. ApJ 426:240

Chiar JE, Adamson AJ, Kerr TH, Whittet DCB. 1995. ApJ 455:234

Chiar JE, Adamson AJ, Whittet DCB. 1996. ApJ 472:665

Chiar JE, Gerakines PA, Whittet DCB, et al. 1998. ApJ 498:716

Chiar JE, Tielens AGGM, Whittet DCB, et al. 2000. ApJ 537:749

Chiar JE, Adamson AJ, Pendleton YJ, et al. 2002. ApJ 570:198

Chiar JE, Pendleton YJ, Allamandola LJ, et al. 2011. ApJ 731:9

Ciesla FJ, Sandford SA. 2012. Science 336:452

Cleeves LI, Bergin EA, Alexander CMOD, et al. 2014. Science 345:1590

Cohen M. 1975. MNRAS 173:279

Cohen M. 1983. ApJL 270:L69

Cox P. 1989. A\&A 225:L1

Cuppen HM, van Dishoeck EF, Herbst E, Tielens AGGM. 2009. A\&A 508:275

Cuppen HM, Penteado EM, Isokoski K, van der Marel N, Linnartz H. 2011. MNRAS 417:2809

Danielson RE, Woolf NJ, Gaustad JE. 1965. ApJ 141:116

Dartois E, Cox P, Roelfsema PR, et al. 1998. A\&A 338:L21

Dartois E, Demyk K, d'Hendecourt L, Ehrenfreund P. 1999. A\&A 351:1066

Dartois E, d'Hendecourt L. 2001. A\&A 365:144

Dartois E, d'Hendecourt L, Thi W, Pontoppidan KM, van Dishoeck EF. 2002. A\&A 394:1057

Dartois E, Thi W-F, Geballe TR, et al. 2003. A\&A 399:1009 
Observations of the Icy Universe; ARAA 53 (2015; accepted), v. 05/05/2015

Dartois E. 2005. Space Science Reviews 119:293

Dartois E. 2006. A\&A 445:959

Debes JH, Weinberger AJ, Schneider G. 2008, ApJL 673:L191

Demyk K, Dartois E, d'Hendecourt L, et al. 1998. A\&A 339:553

Demyk K, Dartois E, Wiesemeyer H, Jones AP, d'Hendecourt L. 2000, A\&A 364:170

d'Hendecourt LB, Allamandola LJ, Grim RJA, Greenberg JM. 1986. A\&A 158:119

Ehrenfreund P, Gerakines PA, Schutte WA, van Hemert MC, van Dishoeck EF. 1996. A\&A 312:263

Ehrenfreund P, Boogert ACA, Gerakines PA, Tielens AGGM, van Dishoeck EF. 1997. A\&A 328:649

Ehrenfreund P, Kerkhof O, Schutte WA, et al. 1999. A\&A 350:240

Elsila J, Allamandola LJ, Sandford SA. 1997. ApJ 479:818

Erickson EF, Knacke RF, Tokunaga AT, Haas MR. 1981. ApJ 245:148

Fayolle EC, Öberg KI, Cuppen HM, Visser R, Linnartz H. 2011. A\&A 529:74

Fedoseev G, Ioppolo S, Zhao D, Lamberts T, Linnartz H. 2015. MNRAS 446:439

Forveille T, Morris M, Omont A, Likkel L. 1987. A\&A 176:L13

Furlan E, McClure M, Calvet N, et al. 2008. ApJS 176:184

Gálvez O, Maté B, Herrero VJ, Escribano R. 2010. ApJ 724:539

Garrod RT, Weaver SLW, Herbst E. 2008. ApJ 682:283

Geballe TR, Baas F, Greenberg JM, Schutte W. 1985. A\&A 146:L6

Gerakines PA, Schutte WA, Greenberg JM, van Dishoeck EF. 1995. A\&A 296:810

Gerakines PA, Schutte W, Ehrenfeund P. 1996. A\&A 312:289

Gerakines PA, Whittet DCB, Ehrenfreund P, et al. 1999. ApJ 522:357

Gerakines PA, Moore MH. 2001a. Icarus 154:372

Gerakines PA, Moore MH, Hudson RL. 2001b. Journal of Geophysical Research 106:33381

Gibb EL, Whittet DCB. 2002. ApJL 566:L113

Gibb EL, Whittet DCB, Boogert ACA, Tielens AGGM. 2004. ApJS 151:35

Gillett FC, Forrest WJ. 1973. ApJ 179:483

Gillett FC, Jones TW, Merrill KM, Stein WA. 1975. A\&A 45:77

Gillett FC, Soifer BT. 1976. ApJ 207:780

Goebel JH. 1983. ApJL 268:L41

Greenberg JM. 1973. In Molecules in the galactic environment, ed. MA Gordon, LE Snyder, Wiley, p. 94.

Greenberg JM, Li A, Mendoza-Gomez CX, et al. 1995. ApJL 455:L177

Grim RJA, Baas F, Greenberg JM, Geballe TR, Schutte W. 1991. A\&A 243:473

Hagen W, Tielens AGGM, Greenberg JM. 1983. A\&A 117:132

Hardegree-Ullman EE, Gudipati MS, Boogert ACA, et al. 2014. ApJ 784:172

Harker D, Bregman J, Tielens AGGM, Temi P, Rank D. 1997. A\&A 324:629 
Herbst E, van Dishoeck EF. 2009. ARAA, 47:427

Holbrook JC, Temi P. 1998. ApJ 496:280

Hollenbach DJ, Werner MW, Salpeter EE. 1971. ApJ 163:165

Hollenbach D, Kaufman MJ, Bergin EA, Melnick GJ. 2009. ApJ 690:1497

Honda M, Inoue AK, Fukagawa M, et al. 2009. ApJL 690:L110

Hough JH, Chrysostomou A, Messinger DW, et al. 1996. ApJ 461:902

Hudgins DM, Sandford SA, Allamandola LJ, Tielens AGGM. 1993. ApJS 86:713

Imanishi M, Maloney PR. 2003. ApJ 588:165

Imanishi M, Dudley CC, Maloney PR. 2006. ApJ 637:114

Ioppolo S, van Boheemen Y, Cuppen HM, van Dishoeck EF, Linnartz H. 2011. MNRAS, 413:2281

Ioppolo S, Sangiorgio I, Baratta GA, Palumbo ME. 2013. A\&A 554:34

Ioppolo S, McGuire BA, Allodi MA, Blake GA. 2014. Faraday Discuss. 168:461

Jenkins EB. 2009. ApJ 700:1299

Jenniskens P. 1993. A\&A 274:653

Keane JV, Boogert ACA, Tielens AGGM, Ehrenfreund P, Schutte WA. 2001a. A\&A 375:L43

Keane JV, Tielens AGGM, Boogert ACA, Schutte WA, Whittet DCB. 2001b. A\&A $376: 254$

Kerr TH, Adamson AJ, Whittet DCB. 1993. MNRAS 262:1047

Kim HJ, Evans NJ II, Dunham MM, Lee J-E, Pontoppidan KM. 2012. ApJ 758:38

Knacke RF, Cudaback DD, Gaustad JE. 1969. ApJ 158:151

Knacke RF, McCorkle S, Puetter RC, Erickson EF, Kraetschmer W. 1982. ApJ 260:141

Knacke RF, McCorkle SM. 1987. AJ 94:972

Knez C, Boogert ACA, Pontoppidan KM, et al. 2005. ApJL 635:L145

Lacy JH, Baas F, Allamandola LJ, et al. 1984. ApJ 276:533

Lacy JH, Carr JS, Evans NJ II, et al. 1991. ApJ 376:556

Lacy JH, Faraji H, Sandford SA, Allamandola LJ. 1998. ApJL 501:L105

Li Q, Liang SL, Li A. 2014. MNRAS 440:L56

Lindblad B. 1935. Nature 135:133

Linnartz H, Bossa JB, Bouwman J, et al. 2011. The Molecular Universe, IAU Symp 280, ed. J Cernicharo, R Bachiller, p. 390. Cambridge, UK: Cambridge Univ. Press

Malfait K, Waelkens C, Waters LBFM, et al. 1998. A\&A 332:L25

Malfait K, Waelkens C, Bouwman J, de Koter A, Waters LBFM. 1999. A\&A 345:181

Mathis JS, Rumpl W, Nordsieck KH. 1977. ApJ 217:425

McClure MK, Espaillat C, Calvet N, et al. 2015, ApJ 799:162

McFadzean AD, Whittet DCB, Bode MF, Adamson AJ, Longmore AJ. 1989. MNRAS 241:873 
Mennella V, Palumbo ME, Baratta GA. 2004. ApJ 615:1073

Merrill KM, Russell RW, Soifer BT. 1976. ApJ 207:763

Moneti A, Cernicharo J, Pardo JR. 2001. ApJL 549:L203

Moore MH, Hudson RL. 1994. A\&AS 103:45

Moore MH, Hudson RL, Gerakines PA. 2001. Spectrochimica Acta Part A: Molecular Spectroscopy, 57:843

Moultaka J, Eckart A, Schödel R, Viehmann T, Najarro F. 2005. A\&A 443:163

Moultaka J, Eckart A, Schödel R. 2009. ApJ 703:1635

Mumma MJ, Charnley SB. 2011. ARAA 49, 471

Muñoz Caro GM, Meierhenrich U, Schutte WA, Thiemann WH-P, Greenberg JM. 2004. A\&A 413:209

Murakawa K, Tamura M, Nagata T. 2000. ApJS 128:603

Noble JA, Fraser HJ, Aikawa Y, Pontoppidan KM, Sakon I. 2013. ApJ 775:85

Öberg KI, Boogert ACA, Pontoppidan KM, et al. 2008. ApJ 678:1032

Öberg KI, Bottinelli S, van Dishoeck EF. 2009a. A\&A 494:L13

Öberg KI, Fayolle EC, Cuppen HM, van Dishoeck EF, Linnartz H. 2009b. A\&A 505:183

Öberg KI, Boogert ACA, Pontoppidan KM, et al. 2011. ApJ 740:109

Oliveira JM, van Loon JT, Chen C-HR, et al. 2009. ApJ 707:1269

Oliveira JM, van Loon JT, Sloan GC, et al. 2011. MNRAS 411:L36

Oliveira JM, van Loon JT, Sloan GC, et al. 2013. MNRAS 428:3001

Omont A, Forveille T, Moseley SH, et al. 1990. ApJL 355:L27

Ootsubo T, Kawakita H, Hamada S, et al. 2012. ApJ 752:15

Ormel CW, Paszun D, Dominik C, Tielens AGGM. 2009. A\&A 502:845

Palumbo ME, Geballe TR, Tielens AGGM. 1997. ApJ 479:839

Palumbo ME, Leto P, Siringo C, Trigilio C. 2008. ApJ 685:1033

Paranicas C, Cooper JF, Garrett HB, et al. 2009. In: Europa, ed. RT Pappalardo, p. 529. Tucson, AZ: University of Arizona Press

Parise B, Simon T, Caux E, et al. 2003. A\&A 410:897

Pendleton YJ, Tielens AGGM, Werner MW. 1990. ApJ 349:107

Pitann J, Hennemann M, Birkmann S, et al. 2011. ApJ 743:93

Pontoppidan KM, Fraser HJ, Dartois E, et al. 2003. A\&A 408:981

Pontoppidan KM, van Dishoeck EF, Dartois E. 2004. A\&A 426:925

Pontoppidan KM, Dullemond CP, van Dishoeck EF, et al. 2005. ApJ 622:463

Pontoppidan KM. 2006. A\&A 453:L47

Pontoppidan KM, Boogert ACA, Fraser HJ, et al. 2008. ApJ 678:1005

Pontoppidan KM, Salyk C, Bergin EA, et al. 2014. In Protostars and Planets VI, University of Arizona Press, ed. H Beuther, R Klessen, C Dullemond, Th Henning, p. $363-385$ 
Poteet CA, Pontoppidan KM, Megeath ST, et al. 2013. ApJ 766:117

Preibisch T, Ossenkopf V, Yorke HW, Henning T. 1993. A\&A 279:577

Raunier S, Chiavassa T, Duvernay F, et al. 2004a. A\&A 416:165

Raunier S, Chiavassa T, Marinelli F, Aycard JP. 2004b. Chem Phys 302:259

Reach WT, Faied D, Rho J, et al. 2009. ApJ 690:683

Sajina A, Spoon H, Yan L, et al. 2009. ApJ 703:270

Sandford SA, Allamandola LJ, Tielens AGGM, Valero GJ. 1988. ApJ 329:498

Sandford SA, Allamandola LJ, Geballe TR. 1993. Science, 262:400

Sandford SA, Bernstein MP, Allamandola LJ, Goorvitch D, Teixeira TCVS. 2001. ApJ 548:836

Schegerer AA, Wolf S. 2010. A\&A 517:87

Schutte WA, Allamandola LJ, Sandford SA. 1993. Icarus 104:118

Schutte WA, Tielens AGGM, Whittet DCB, et al. 1996. A\&A 315:L333

Schutte WA, Boogert ACA, Tielens AGGM, et al. 1999. A\&A 343:966

Schutte WA, Khanna RK. 2003. A\&A 398:1049

Seale JP, Looney LW, Chu Y-H, et al. 2009. ApJ 699:150

Seale JP, Looney LW, Chen C-HR, Chu Y-H, Gruendl RA. 2011. ApJ 727:36

Sellgren K, Smith RG, Brooke TY. 1994. ApJ 433:179

Shimonishi T, Onaka T, Kato D, et al. 2008. ApJL 686:L99

Shimonishi T, Onaka T, Kato D, et al. 2010. A\&A 514:12

Shuping RY, Snow TP, Chiar JE, Kerr T. 2000. ApJ 529:932

Skinner CJ, Tielens AGGM, Barlow MJ, Justtanont K. 1992. ApJL 399:L79

Smith RG, Sellgren K, Tokunaga AT. 1988. ApJ 334:209

Smith RG, Sellgren K, Tokunaga AT. 1989. ApJ 344:413

Smith RG. 1991. MNRAS 249:172

Smith RG, Sellgren K, Brooke TY. 1993. MNRAS 263:749

Smith RG, Charnley SB, Pendleton YJ, et al. 2011. ApJ 743:131

Soifer BT, Puetter RC, Russell RW, et al. 1979. ApJL 232:L53

Soifer BT, Willner SP, Rudy RJ, Capps RW. 1981. ApJ 250:631

Spoon HWW, Koornneef J, Moorwood AFM, Lutz D, Tielens AGGM. 2000. A\&A $357: 898$

Spoon HWW, Moorwood AFM, Pontoppidan KM, et al. 2003. A\&A 402:499

Spoon HWW, Armus L, Cami J, et al. 2004. ApJS 154:184

Sturm E, Lutz D, Tran D, et al. 2000. A\&A 358:481

Sylvester RJ, Kemper F, Barlow MJ, et al. 1999. A\&A 352:587

Taban IM, Schutte WA, Pontoppidan KM, van Dishoeck EF. 2003. A\&A 399:169

Tanaka M, Sato S, Nagata T, Yamamoto T. 1990. ApJ 352:724

Terada H, Tokunaga AT, Kobayashi N, et al. 2007. ApJ 667:303 
Terada H, Tokunaga AT. 2012a. ApJ 753:19

Terada H, Tokunaga AT, Pyo T-S, et al. 2012b. AJ 144:175

Tielens AGGM, Hagen W. 1982. A\&A 114:245

Tielens AGGM, Allamandola LJ. 1987a. In: Physical Processes in Interstellar Clouds, ed. GE Morfill, M Scholer, NATO ASIC Proc. 210, p. 333

Tielens AGGM, Allamandola LJ. 1987b. In: Interstellar Processes, ed. DJ Hollenbach, HA Thronson, p. 414. Dordrecht: Reidel,

Tielens AGGM, Tokunaga AT, Geballe TR, Baas F. 1991. ApJ 381:181

Theulé P, Duvernay F, Danger G, et al. 2013. Advances in Space Research 52:1567

Thi W-F, van Dishoeck EF, Dartois E, et al. 2006. A\&A 449:251

van Broekhuizen FA, Keane JV, Schutte WA. 2004. A\&A 415:425

van Broekhuizen FA, Pontoppidan KM, Fraser HJ, van Dishoeck EF. 2005. A\&A $441: 249$

van de Hulst H. 1946. Recherches Astronomiques de l'Observatoire d'Utrecht 11:2

van den Ancker ME, Bouwman J, Wesselius PR, et al. 2000. A\&A 357:325

Vandenbussche B, Ehrenfreund P, Boogert ACA, et al. 1999. A\&A 346:L57

van der Tak FFS, van Dishoeck EF, Evans NJ II, Blake GA. 2000. ApJ 537:283

van Loon JT, Oliveira JM, Gordon KD, Sloan GC, Engelbracht CW. 2010, AJ 139:1553

van Thiel M, Becker ED, Pimentel GC. 1957. JChPh 27:486

Vasyunina T, Vasyunin AI, Herbst E, et al. 2014. ApJ 780:85

Visser R, van Dishoeck EF, Doty SD, Dullemond CP. 2009. A\&A 495:881

Vuong MH, Montmerle T, Grosso N, et al. 2003. A\&A 408:581

Warren SG, Brandt RE. 2008. Journal of Geophysical Research (Atmospheres) 113:14220

Watanabe N, Kouchi A. 2002. ApJL 571:173

Watson WD, Salpeter EE. 1972. ApJ 174:321

Whittet DCB, Bode MF, Baines DWT, Longmore AJ, Evans A. 1983. Nature 303:218

Whittet DCB, McFadzean AD, Longmore AJ. 1985. MNRAS 216:45

Whittet DCB, Gerakines PA, Tielens AGGM, et al. 1998. ApJL 498:L159

Whittet DCB, Gerakines PA, Hough JH, Shenoy SS. 2001. ApJ 547:872

Whittet DCB, Shenoy SS, Bergin EA, et al. 2007, ApJ 655:332

Whittet DCB, Cook AM, Chiar JE, et al. 2009. ApJ 695:94

Whittet DCB. 2010. ApJ 710:1009

Whittet DCB. 2011a. In: Astronomical Polarimetry 2008: Science from Small to

Large Telescopes, ed. P Bastien, N Manset, DP Clemens, N St-Louis, ASP Conf.

Ser. 449 , p. 93

Whittet DCB, Cook AM, Herbst E, Chiar JE, Shenoy SS. 2011b. ApJ 742:28

Whittet DCB, Poteet CA, Chiar JE, et al. 2013. ApJ 774:102

Yamagishi M, Kaneda H, Ishihara D, et al. 2013. ApJL 773:L37

Zasowski G, Kemper F, Watson DM, et al. 2009. ApJ 694:459 